\title{
Multi-species turbulent mixing under supercritical-pressure conditions: modelling, direct numerical simulation and analysis revealing species spinodal decomposition
}

\author{
Enrica Masi ${ }^{1}$, Josette Bellan ${ }^{1,2} \dagger$, Kenneth G. Harstad ${ }^{2}$ and Nora A. Okong'o ${ }^{2}$ \\ ${ }^{1}$ California Institute of Technology, Pasadena, CA 91125, USA \\ ${ }^{2}$ Jet Propulsion Laboratory, California Institute of Technology, Pasadena, CA 91109, USA
}

(Received 5 December 2012; revised 23 January 2013; accepted 28 January 2013; first published online 19 March 2013)

A model is developed for describing mixing of several species under high-pressure conditions. The model includes the Peng-Robinson equation of state, a full massdiffusion matrix, a full thermal-diffusion-factor matrix necessary to incorporate the Soret and Dufour effects and both thermal conductivity and viscosity computed for the species mixture using mixing rules. Direct numerical simulations (DNSs) are conducted in a temporal mixing layer configuration. The initial mean flow is perturbed using an analytical perturbation which is consistent with the definition of vorticity and is divergence free. Simulations are performed for a set of five species relevant to hydrocarbon combustion and an ensemble of realizations is created to explore the effect of the initial Reynolds number and of the initial pressure. Each simulation reaches a transitional state having turbulent characteristics and most of the data analysis is performed on that state. A mathematical reformulation of the flux terms in the conservation equations allows the definition of effective species-specific Schmidt numbers $(S c)$ and of an effective Prandtl number $(P r)$ based on effective speciesspecific diffusivities and an effective thermal conductivity, respectively. Because these effective species-specific diffusivities and the effective thermal conductivity are not directly computable from the DNS solution, we develop models for both of these quantities that prove very accurate when compared with the DNS database. For two of the five species, values of the effective species-specific diffusivities are negative at some locations indicating that these species experience spinodal decomposition; we determine the necessary and sufficient condition for spinodal decomposition to occur. We also show that flows displaying spinodal decomposition have enhanced vortical characteristics and trace this aspect to the specific features of high-density-gradient magnitude regions formed in the flows. The largest values of the effective speciesspecific $S c$ numbers can be well in excess of those known for gases but almost two orders of magnitude smaller than those of liquids at atmospheric pressure. The effective thermal conductivity also exhibits negative values at some locations and the effective $\operatorname{Pr}$ displays values that can be as high as those of a liquid refrigerant. Examination of the equivalence ratio indicates that the stoichiometric region is thin and coincides with regions where the mixture effective species-specific Lewis number values are well in excess of unity. Very lean and very rich regions coexist in the vicinity of the stoichiometric region. Analysis of the dissipation indicates that it is

$\dagger$ Email address for correspondence: josette.bellan@jpl.nasa.gov 
dominated by mass diffusion, with viscous dissipation being the smallest among the three dissipation modes. The sum of the heat and species (i.e. scalar) dissipation is functionally modelled using the effective species-specific diffusivities and the effective thermal conductivity. Computations of the modelled sum employing the modelled effective species-specific diffusivities and the modelled effective thermal conductivity shows that it accurately replicates the exact equivalent dissipation.

Key words: mixing, turbulent mixing

\section{Introduction}

Mixing of several species under high-pressure (high- $p$ ) conditions is encountered in a variety of practical systems used for automotive, aeronautics and astronautics propulsion. These systems include diesel, homogeneous charge compression ignition (HCCI), gas turbine and liquid rocket propulsion engines; in all of these devices $p$ is generally larger than the critical pressure, $p_{c}$ (subscript $c$ denotes the critical state), of either fuel or oxidizer. In the combustion chamber of each of these systems, a chemical species or a complex fuel is injected into another fluid (which can be an oxidizer) which is at $p>p_{c}$. Thus, high- $p$ mixing is a precursor to combustion. The evolution of the mixture during preignition, a phenomenon occurring at relatively low-temperature (with respect to full combustion) and high- $p$ conditions, has been experimentally demonstrated to be a chemical-radical-deficient process (Fotache, Kreutz \& Law 1997; Egolfopoulos \& Dimotakis 1998), a fact which necessarily implies that molecular mixing is crucial to autoignition; details of molecular mixing strongly influence the inception and development of autoignition. This preignition is chemically dominated by pyrolysis, a process in which the fuel gradually decomposes into its lighter species which mix with the remaining fuel and oxidizer. During combustion, since the reaction is not uniformly distributed, reaction products evolving at some locations may also mix with fuel and oxidizer at other locations. Thus, turbulent mixing of several chemical species under high- $p$ conditions is a crucial topic of study relevant to a wide range of combustion devices and operating conditions.

The investigation of the fundamental processes occurring during $p>p_{c}$ mixing of several species is best studied through direct numerical simulation (DNS) because well-conducted DNSs present a unique opportunity to explore details which are only approximated when using other computational methods (e.g. large eddy simulation (LES)). Thus, the focus of the present study is to conduct DNSs of multi-species mixing under $p>p_{c}$ conditions and analyse the results. First, we wish to elucidate some of the similarities and differences between past DNS studies of $p>p_{c}$ binaryspecies mixing (Okong'o \& Bellan 2002a; Okong'o, Harstad \& Bellan 2002) and the present more complex and realistic situation. The most important difference between the binary-species studies and the present one is in the new physics embedded in the transport properties and in the real-gas thermodynamic complexities when several species are involved. Because of the strong coupling between dynamics and thermodynamics for $p>p_{c}$ flows, these new aspects are expected to lead to new insights into the particular care with which simulations of such practical flows must be approached. For example, the mixing of two species involves a single Schmidt number, $S c \equiv \mu /\left(\rho \alpha_{D} \mathscr{D}\right)$ (where $\mu$ is the viscosity coefficient, $\rho$ is the mass density, $\alpha_{D}$ is the mass-diffusion factor computable from the equation of state (EOS) 
(Okong'o \& Bellan 2002a; Okong'o et al. 2002) and $\mathscr{D}$ is the diffusion coefficient), whereas the mixing of several species involves many $S c$ numbers; this fact has physical consequences which translate into computational requirements. That is, because the relationship between the Kolmogorov scale, $\eta_{K}$, and the Batchelor scale, $\eta_{B}$, is $\eta_{B}=\eta_{K} S c^{-0.5}$, only if $S c \lesssim 1$ (gas-like values) is the relationship $\eta_{B} \gtrsim \eta_{K}$ ensured, and thus only then is the resolution for the flow dynamics sufficient for describing species mixing; however, for multiple species, a single $S c$ is not expected to portray the characteristic scales associated with mixing of all species. It would be desirable to define a single $S c$ for each species, but such definition is absent so far. Furthermore, because supercritical fluids have properties intermediate between liquid and gas, even if a species-specific $S c$ were defined, it cannot be ensured that $S c \lesssim 1$ holds for all $S c$ numbers at all times and locations; this fact may impose additional computational demands in DNSs to resolve the smallest dissipation-relevant scales due to mixing. There is a similar concern about resolving the thermal scales, $\eta_{\theta}$, since $\eta_{\theta}=\eta_{K} P^{-0.5}$ (Goto \& Kida 1999). Second, we wish to explore the impact of the coupled thermodynamics and transport properties on the species, temperature and equivalence ratio $(E R)$ distribution. In particular, we wish to inquire whether the stoichiometric region is thin, so that if this feature further holds for reactive cases the flow may be in the 'flamelet' regime (Williams 1975). With reaction, the stoichiometric region may be more distorted and thicker compared with that obtained during mixing, but if it is not thin without reaction, the possibility of being thin when reaction occurs is remote. With a potentially thin flame, one could conceivably use the Peters (2000) flamelet model if the equations can be reformulated to fit that model. Finally, we are interested in understanding the major fundamental aspects of multiplespecies mixing under supercritical $p$ as a precursor to undertaking reactive-flow studies in the future.

The model describing species mixing is presented in $\S 2$. The species governing equations are no longer in the molar form of the past (Okong'o \& Bellan 2002a), and instead have been restructured in mass-fraction format and the system has been manipulated to solve for a number of independent species. The intricacies of the multiple-species transport properties is then described in $\S 3$. To the best of the authors' knowledge, only Giovangigli, Matuszewsky \& Dupoirieux (2011) and Giovangigli \& Matuszewsky (2012) have modelled transport properties in such detail in studies focused on steady one-dimensional laminar flames. The physical configuration, boundary conditions and initial conditions are presented in $\S 4$ and the numerical method for solving the equations using DNS is explained in $\S 5$. A database of eight DNS realizations is constructed for a simple system of species relevant to combustion, fuel ( $n$-heptane, $\mathrm{C}_{7} \mathrm{H}_{16}$; subscript Hep), oxygen $\left(\mathrm{O}_{2}\right.$; subscript $\left.O x y\right)$, nitrogen $\left(\mathrm{N}_{2}\right.$; subscript $\left.N\right)$, water vapour $\left(\mathrm{H}_{2} \mathrm{O}\right.$; subscript Wat) and carbon dioxide $\left(\mathrm{CO}_{2}\right.$; subscript $\left.\mathrm{Car}\right)$, and results are illustrated and discussed in $\S 6$ highlighting the effect of thermodynamics and transport properties. The choice of $\mathrm{H}_{2} \mathrm{O}$ and $\mathrm{CO}_{2}$ has practical applications to HCCI engines in which fuel is mixed with recirculated exhaust gas that is compressed in the combustion chamber before ignition. After discussing the evolution of the flow in $\S 6.1$ we examine in $\S 6.2$ the transitional states achieved for each simulation. Most significantly, we show in $\S 6.2 .2$ that it is possible to mathematically define effective species-specific diffusivities and an effective thermal conductivity leading to effective species-specific $S c$ numbers, $S c_{\alpha, \text { eff }}$, and an effective Prandtl number, $P r_{\text {eff }}$, and we propose a model for computing these numbers; comparisons of the model with the database shows that the model is very accurate. Both effective $S c$ and $P r$ number values indicate that the flow possesses characteristics 
far from those of gases, that some aspects are very much like those of liquids, that the mixture is non-ideal and that some species experience spinodal decomposition under certain conditions; we determine the necessary and sufficient conditions for spinodal decomposition to occur. Also, we find in $\$ 6.2 .3$ that the stoichiometric region is thin and that the fluid mixture in that region has effective species-specific Lewis number values larger than unity. As a way to gather the remaining information relevant to transport properties and their effect on the flow, in $\S 6.3$ we examine the dissipation and its various contributions. A summary and conclusions are offered in $\$ 7$ with special emphasis on the implications for simulations of $p>p_{c}$ reactive flows.

\section{Governing equations}

\subsection{Differential conservation equations}

The conservation equations derived in detail elsewhere (Okong'o \& Bellan 2002a) are

$$
\begin{gathered}
\frac{\partial \rho}{\partial t}+\frac{\partial}{\partial x_{j}}\left[\rho u_{j}\right]=0, \\
\frac{\partial}{\partial t}\left(\rho u_{i}\right)+\frac{\partial}{\partial x_{j}}\left[\rho u_{i} u_{j}+p \delta_{i j}-\sigma_{i j}\right]=0, \\
\frac{\partial}{\partial t}\left(\rho e_{t}\right)+\frac{\partial}{\partial x_{j}}\left[\left(\rho e_{t}+p\right) u_{j}-u_{i} \sigma_{i j}+q_{j}\right]=0, \\
\frac{\partial}{\partial t}\left(\rho Y_{\alpha}\right)+\frac{\partial}{\partial x_{j}}\left[\rho Y_{\alpha} u_{j}+J_{\alpha j}\right]=0,
\end{gathered}
$$

where $\alpha \in[1, N-1], t$ denotes the time, $x$ is a Cartesian coordinate, subscripts $i$ and $j$ refer to the spatial coordinates, $u_{i}$ is the velocity, $e_{t}=e+u_{i} u_{i} / 2$ is the total energy (i.e. internal energy, $e$, plus kinetic energy), $Y_{\alpha}$ is the mass fraction of species $\alpha, N$ is the number of species and $\sigma_{i j}$ is the Newtonian viscous stress tensor

$$
\sigma_{i j}=\mu\left(2 S_{i j}-\frac{2}{3} S_{k k} \delta_{i j}\right), \quad S_{i j}=\frac{1}{2}\left(\frac{\partial u_{i}}{\partial x_{j}}+\frac{\partial u_{j}}{\partial x_{i}}\right),
$$

where $\mu$ is the viscosity and $J_{\alpha j}$ and $q_{j}$ are the $j$-direction species- $\alpha$ mass flux and heat flux, respectively.

Because of the different constitutive relations used for expressing $\boldsymbol{J}_{\alpha}$ and $\boldsymbol{q}$, equations (2.1)-(2.4) under high- $p$ conditions have ultimately a different form from their equivalent form used under atmospheric- $p$ conditions. The $\boldsymbol{J}_{\alpha}$ and $\boldsymbol{q}$ expressions used here are based on the full matrices of mass-diffusion coefficients and thermaldiffusion factors derived by Harstad \& Bellan (2004a). In order to ensure global mass conservation, we choose to consider a set of independent $(N-1)$ species equations rather than the set of $N$ dependent species; in these $(N-1)$ equations, the original molar-fraction fluxes and the heat flux were rewritten to account for only $(N-1)$ gradients. The final form is

$$
\begin{gathered}
\boldsymbol{J}_{\alpha}=-\rho\left[Y_{\alpha}\left(D_{T, \alpha}\right) \frac{\nabla T}{T}+Y_{\alpha}\left(D_{p, \alpha}\right) \frac{\nabla p}{p}+\sum_{\beta=1}^{N-1}\left(D_{\alpha \beta}^{\prime} \frac{m_{\alpha}}{m_{\beta}}\right) \nabla Y_{\beta}\right], \\
\boldsymbol{q}=-\lambda \nabla T+\sum_{\alpha=1}^{N-1} \boldsymbol{J}_{\alpha}\left[\left(\frac{h_{\alpha}}{m_{\alpha}}-\frac{h_{N}}{m_{N}}\right)-R_{u} T\left(\frac{\bar{\alpha}_{T, \alpha}^{b}}{m_{\alpha}}-\frac{\bar{\alpha}_{T, N}^{b}}{m_{N}}\right)\right]
\end{gathered}
$$


where

$$
\begin{gathered}
D_{T, \alpha}=-\sum_{\beta=1}^{N} \bar{\alpha}_{T, \beta}^{b} \mathbb{D}_{\beta \alpha}, \quad D_{p, \alpha}=\frac{p}{R_{u} T} \sum_{\beta=1}^{N} v_{\beta} \mathbb{D}_{\beta \alpha}, \\
D_{\alpha \gamma}=\sum_{\beta=1}^{N} \mathbb{D}_{\alpha \beta} \alpha_{D \beta \gamma}, \\
\bar{\alpha}_{T, \alpha}^{b}=\sum_{\beta=1}^{N} X_{\beta} \alpha_{T, \beta \alpha}^{b} \\
D_{\alpha \beta}^{\prime}=D_{\alpha \beta}-\left(1-\frac{m_{\beta}}{m_{N}}\right)\left(\sum_{\gamma=1}^{N-1} D_{\alpha \gamma} X_{\gamma}\right) .
\end{gathered}
$$

Here, $X_{\alpha}=Y_{\alpha} m / m_{\alpha}$ represents the molar fraction, $T$ is the temperature, $m_{\alpha}$ is the species molar mass, $m$ is the mixture molar mass, $m=\sum_{\gamma=1}^{N} m_{\gamma} X_{\gamma}, v_{\alpha}=$ $\left(\partial v / \partial X_{\alpha}\right)_{T, p, X_{\beta}(\beta \neq \alpha)}$ is the partial molar volume, where the molar volume is $v=1 / n$ and $n=\rho / m$ is the molar density, $h_{\alpha}=\left(\partial h / \partial X_{\alpha}\right)_{T, p, X_{\beta}(\beta \neq \alpha)}$ is the partial molar enthalpy, where the molar enthalpy is $h=G-T(\partial G / \partial T)_{p, X}$ with $G$ being the Gibbs energy, $R_{u}$ is the universal gas constant, $D_{\alpha \gamma}$ are the pairwise mass-diffusion coefficients, $\alpha_{T, \alpha \beta}^{b}$ are the binary thermal diffusion factors and $\lambda$ is the thermal conductivity. The mass-diffusion factors, $\alpha_{D \alpha \beta}$, are calculated from thermodynamics as

$$
\begin{gathered}
\alpha_{D \alpha \beta} \equiv \frac{1}{R_{u} T} X_{\alpha} \frac{\partial \mu_{\alpha}}{\partial X_{\beta}}=\left(\delta_{\alpha \beta}-\delta_{\alpha N}\right)+X_{\alpha}\left(R_{\alpha \beta}-R_{\alpha N}\right), \quad 1 \leqslant \alpha \leqslant N, 1 \leqslant \beta \leqslant N-1 \\
R_{\alpha \beta} \equiv \frac{\partial \ln \gamma_{\alpha}}{\partial X_{\beta}}, \quad 1 \leqslant \alpha \leqslant N, \quad 1 \leqslant \beta \leqslant N
\end{gathered}
$$

where $\mu_{\alpha}$ is the chemical potential of species $\alpha$ written in terms of $N-1$ species; $\gamma_{\alpha} \equiv \varphi_{\alpha} / \varphi_{\alpha}^{o}$ where $\varphi$ is the fugacity coefficient written in terms of $N$ species and the superscript $o$ denotes the pure $\left(X_{\alpha}=1\right)$ limit. Matrix elements $\mathbb{D}_{\beta \gamma}$ are the solution of the mixing rules equations (Harstad \& Bellan 2004a)

$$
\sum_{\beta=1}^{N}\left[\delta_{\alpha \beta}-\left(1-\delta_{\alpha \beta}\right) X_{\beta} \frac{\overline{\mathscr{D}}_{\alpha}}{\mathscr{D}_{\alpha \beta}^{b}}\right] \frac{\mathbb{D}_{\beta \gamma}}{X_{\beta}}=\overline{\mathscr{D}}_{\alpha} \frac{\left(\delta_{\alpha \gamma}-Y_{\alpha}\right)}{X_{\alpha}}
$$

where

$$
\overline{\mathscr{D}}_{\alpha}=1 / \sum_{\substack{\beta=1 \\ \beta \neq \alpha}}^{N}\left(\frac{X_{\beta}}{\mathscr{D}_{\alpha \beta}^{b}}\right) .
$$

Solutions for $\mathbb{D}_{\beta \gamma}$ may be obtained by an approximate inversion (Ern \& Giovangigli 1998) as follows:

$$
\begin{aligned}
& \mathbb{D}_{\beta \gamma} \simeq X_{\beta} \mathbb{D}_{\beta \gamma}^{(1)}, \\
& \mathbb{D}_{\alpha \beta}^{(1)}=\frac{\left(1+Y_{\alpha}\right)}{X_{\alpha}} \mathscr{D}_{\alpha}^{*} \delta_{\alpha \beta}+\left(1-\delta_{\alpha \beta}\right) \frac{\mathscr{D}_{\alpha}^{*} \mathscr{D}_{\beta}^{*}}{\mathscr{D}_{\alpha \beta}^{b}}-\left(\sigma_{\alpha} \mathscr{D}_{\alpha}^{*}+\sigma_{\beta} \mathscr{D}_{\beta}^{*}\right)+\sum_{\gamma=1}^{N}\left(Y_{\gamma} \sigma_{\gamma} \mathscr{D}_{\gamma}^{*}\right),
\end{aligned}
$$




$$
\begin{aligned}
\mathscr{D}_{\alpha}^{*} & =\left(1-Y_{\alpha}\right) \overline{\mathscr{D}}_{\alpha}, \\
\sigma_{\alpha} & =\frac{m_{\alpha}}{m}\left(1+Y_{\alpha}\right)+\sum_{\substack{\beta=1 \\
\beta \neq \alpha}}^{N} Y_{\beta} \frac{\mathscr{D}_{\beta}^{*}}{\mathscr{D}_{\alpha \beta}^{b}}
\end{aligned}
$$

where $\mathscr{D}_{\alpha \beta}^{b}$ is the full approximation binary-diffusion matrix. This method leads to a singularity when the mixture is composed of only one species (as, for instance, in pure fuel zones). In that case (2.15) is no longer used and the diffusion coefficients are evaluated by means of the binary-diffusion matrix, by setting $\mathscr{D}_{\alpha}^{*}=\mathscr{D}_{\alpha N}^{b}$, where $N$ represents the index associated with the solvent. This method was tested against an exact Gauss inversion (not shown) and it gave the same results, with an additional gain in computational time. Defining $\mathscr{D}_{\alpha \beta}$ as the first approximation of the binary diffusion matrix and realizing that the deviation of the ratio $\mathscr{D}_{\alpha \beta}^{b} / \mathscr{D}_{\alpha \beta}$ from unity is comparable to uncertainties in binary diffusion coefficients values (Harstad \& Bellan 2004a), we assume $\mathscr{D}_{\alpha \beta}^{b}=\mathscr{D}_{\alpha \beta}$. The computation of $\mathscr{D}_{\alpha \beta}$ and $\alpha_{T, \alpha \beta}^{b}$ is described in $\S 3$ along with the other transport properties.

\subsection{The equation of state}

Equations (2.1)-(2.4) are coupled with the Peng-Robinson (PR) EOS

$$
p=\frac{R_{u} T}{\left(v_{P R}-b_{m i x}\right)}-\frac{a_{\text {mix }}}{\left(v_{P R}^{2}+2 b_{m i x} v_{P R}-b_{m i x}^{2}\right)}
$$

from which $T$ and $p$ are obtained as an iterative solution which satisfies both values of $\rho$ and of $e$, as obtained from the conservation equations (Okong'o et al. 2002). Here $v_{P R}$ is the molar PR volume, and $v=v_{P R}+v_{s}$ where $v_{s}$ is the volume shift introduced so as to improve the accuracy of the PR EOS at high $p ; a_{\text {mix }}$ and $b_{\text {mix }}$ are functions of $T$ and $X_{i}$ (see appendix A). The $v_{s}$ computation was explained in detail elsewhere (Okong'o et al. 2002).

\section{Transport properties}

Unlike for atmospheric- $p$ flows where three transport properties are generally sufficient (viscosity, diffusivity and thermal conductivity), for high- $p$ conditions there are four relevant transport properties.

\subsection{Mixture viscosity}

To compute the individual species viscosity, $\mu_{\alpha}^{v i s}$, the Lucas method (Reid, Prausnitz $\&$ Polling 1987) has been selected due to its high-p-accuracy capabilities. To compute the mixture physical viscosity, $\mu_{p h}$, the Wilke method (Reid et al. 1987) is utilized providing

$$
\begin{aligned}
\mu_{p h} & =\sum_{\alpha=1}^{N} X_{\alpha} \omega_{\alpha}^{M} \mu_{\alpha}^{v i s} \\
\left(\omega_{\alpha}^{M}\right)^{-1} & =\sum_{\beta=1}^{N} \phi_{\alpha \beta} X_{\beta} \\
\phi_{\alpha \beta} & =\frac{\left[1+\left(\mu_{\alpha}^{v i s} / \mu_{\beta}^{v i s}\right)^{1 / 2}\left(m_{\beta} / m_{\alpha}\right)^{1 / 4}\right]^{2}}{\left[8\left(1+m_{\alpha} / m_{\beta}\right)\right]^{1 / 2}}
\end{aligned}
$$


where $\omega_{\alpha}^{M}$ are weighting factors (Reid et al. 1987). The Wilke method is chosen here for the mixing rules because it is a subset of the Wassiljewa-Mason-Saxena method (Reid et al. 1987) for computing thermal conductivities (see §3.2) and provides consistency between these two computations.

We distinguish between $\mu_{p h}$, the reference viscosity $\mu_{R}$ defined in $\S 4$ and the computational viscosity $\mu$ used to enable resolution to scales of $O\left(\eta_{K}\right)$, as explained in $\S 4$.

\subsection{Mixture thermal conductivity}

To compute the physical mixture thermal conductivity, $\lambda_{p h}$, first the species conductivities $\lambda_{\alpha}$ are calculated using the Stiel-Thodos method (Reid et al. 1987) because of its validity at high $p$ through the inclusion of an excess function, and then the Wassiljewa-Mason-Saxena method is utilized to compute $\lambda_{p h}$ from $\lambda_{\alpha}$ as

$$
\lambda_{p h}=\sum_{\gamma=1}^{N} X_{\alpha} \omega_{\alpha}^{Q} \lambda_{\alpha}
$$

where according to the assumption made by Reid et al. (1987)

$$
\omega_{\alpha}^{Q}=\omega_{\alpha}^{M} .
$$

In $\S 4$ we explain how a scaled thermal conductivity, $\lambda$, is computed that is used to perform DNSs.

\subsection{Binary mass diffusivities}

Matrix elements $\mathscr{D}_{\alpha \gamma}^{b}$ are the building blocks of $\mathbb{D}_{\alpha \gamma}$ and ultimately of $D_{\alpha \gamma}^{\prime}$. To compute $\mathscr{D}_{\alpha \gamma}^{b}=\mathscr{D}_{\alpha \gamma}$, we adopt the method of Harstad \& Bellan (2004b) which gives (in cgs units)

$$
n \mathscr{D}_{\alpha \gamma}=2.81 \times 10^{-5} \frac{f_{D, \alpha \gamma}(T)}{r_{D} v_{c, \alpha \gamma}^{2 / 3}}\left[\left(\frac{1}{m_{\alpha}}+\frac{1}{m_{\gamma}}\right) T\right]^{1 / 2}
$$

where $f_{D, \alpha \gamma}(T)$ is generically defined for each matrix element as $f_{D}(T) \equiv\left(T_{\text {red }}\right)^{s}$ with $\ln s=\sum_{\zeta=0}^{5} a_{\zeta}^{s}\left(\ln T_{\text {red }}\right)^{\zeta}$ where the $a^{s}$ vector has elements $\{-0.84211,-0.32643$, $-0.10053,0.07747,0.0127,-0.00995\}$ and $r_{D}$ is a constant $O(1)$ which provides an empirical adjustment for the specifics of the binary collisional interactions of a selected pair of species to adjust the kinetic theory computations so as to match experimental data. Here $T_{r e d, \alpha \gamma} \equiv T / T_{c, \alpha \gamma}$ with $T_{c, \alpha \gamma}$ defined in appendix A; $v_{c, \alpha \gamma}$ is defined in appendix A as well. Values of $r_{D}$ are listed elsewhere (Harstad \& Bellan 2004b) for species pairs relevant to combustion.

\subsection{Binary thermal diffusion factors}

According to Harstad \& Bellan (2004a)

$$
\begin{aligned}
\alpha_{T, \alpha \gamma}^{b} & =\zeta_{\alpha \gamma} \frac{\left(m_{\alpha} \omega_{\gamma}^{T}-m_{\gamma} \omega_{\alpha}^{T}\right)}{\left(m_{\alpha}+m_{\gamma}\right) \mathscr{D}_{\alpha \gamma}} \\
\omega_{\alpha}^{T} & =\frac{\omega_{\alpha}^{Q} \lambda_{\alpha}}{R_{u} n}, \quad \zeta_{\alpha \gamma}=\frac{6}{5} C_{\alpha \gamma}^{*}-1
\end{aligned}
$$

where $\omega_{\alpha}^{Q}$ is computed from (3.2) and (3.5), and $C_{\alpha \gamma}^{*}$ is given by Hirshfelder, Curtis $\&$ Bird (1964) and is function of a normalized temperature including the characteristic 
molecular interaction potential (Harstad \& Bellan 2004a). This computation of $\alpha_{T, \alpha \gamma}^{b}$ is based on kinetic theory and is thus valid for low-to-moderate pressures.

\section{Configuration, boundary conditions and initial conditions}

The configuration is that of a temporal mixing layer with periodic boundary conditions in the streamwise $\left(x_{1}\right)$ and spanwise $\left(x_{3}\right)$ directions and non-reflecting boundary conditions in the cross-stream $\left(x_{2}\right)$ direction (Okong'o \& Bellan 2002b). The domain size in the streamwise $\left(L_{1}\right)$ and spanwise $\left(L_{3}\right)$ directions is such that it accommodates initially four vortices associated with the wavelengths $\lambda_{1}$ and $\lambda_{3}$ of perturbations (see appendix B.2), respectively. The cross-stream domain size $\left(L_{2}\right)$ is large enough so that there is no interference of the mixing region with the domain boundaries.

\subsection{Computational-transport-property selection}

The initial Reynolds number was defined as $\operatorname{Re}_{0} \equiv\left[0.5\left(\rho_{U}+\rho_{L}\right) \Delta U_{0} \delta_{\omega, 0}\right] / \mu_{R}$ where $\rho_{U}$ and $\rho_{L}$ are mixture initial densities, with subscripts $U$ and $L$ labelling the upper and lower streams, respectively; $\delta_{\omega, 0}=\Delta U_{0} /\left(\partial u_{0} / \partial x_{2}\right)_{\max }$ is the initial vorticity thickness computed by using $u_{0}$ which is the $\left(x_{1}, x_{3}\right)$ planar average of the initial velocity in the streamwise direction; $\Delta U_{0}=U_{U}-U_{L}$ is the initial velocity difference across the layer; and as already stated, $\mu_{R}$ is a reference viscosity. For the previous binarymixing simulations (Okong'o \& Bellan 2002a), an initial $\mu_{R}$ was obtained from the chosen $R e_{0}$ value, and then used as a normalizing factor for the viscosity which was expressed as a power of $T$ normalized by an averaged temperature $T_{0}=\left(T_{U}+T_{L}\right) / 2$. The viscosity was then used for computing $P r$ and $S c$ through correlations involving $Y_{\alpha}$ and/or $T$. In the present study, first a physical initial mixture viscosity $\mu_{p h, 0}$ is computed based on the physical initial species viscosities (\$3.1), then a reference value $\mu_{R}$ is obtained from the chosen $R e_{0}$ and finally a factor $\mathscr{F} \equiv \mu_{R} / \mu_{p h, 0}$ is defined. All transport properties computed during the simulation are then scaled by $\mathscr{F}$, a procedure which allows the computation of accurate dimensionless numbers. The value of $\mathscr{F}$ was computed at the initial time, for the initial constant $p_{0}$ and $T_{0}$, using a mixture composed of five species, each species being averaged over the entire domain. In this way, a unique $\mathscr{F}$ value is employed in the computational domain. As an example, for $R e_{0}=600, \mathscr{F}$ was found to be $O\left(10^{4}\right)$; see table 1 . This scaling satisfies the Batchelor (1999) principle of flow similarity stating that flow characteristics only depend upon non-dimensional numbers rather than individual transport properties.

\subsection{Initial profiles}

The initial profiles are composed of mean quantities upon which perturbations are imposed. The choice of mean profiles is usually based on an error function and a similarity analysis; in the past, the perturbations have been related to the stability of the layer, but their specification is rather ad hoc (Okong'o \& Bellan 2004). In appendix B, we show that it is possible to analytically derive a perturbation for initializing the computation. Details about both the mean flow and the analytical perturbation are provided in appendix B and these analytical expressions are here used as initial profiles.

\section{Numerical method}

The differential equations of $\S 2.1$ combined with the EOS described in $\S 2.2$, using the transport coefficient models presented in $\S 3$, were numerically solved 


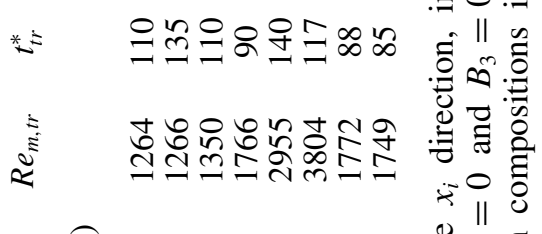

호호

它草总

彥

$\Xi 0 . \Omega$

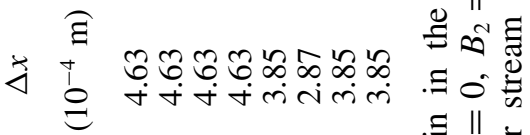

: $\|$

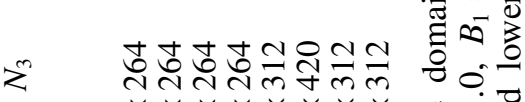

$x \quad x \times x \times x \times x \times$

之 $\quad$ m

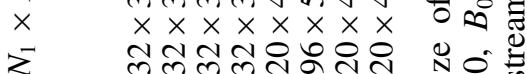

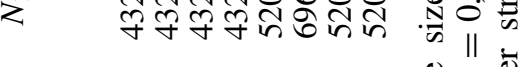

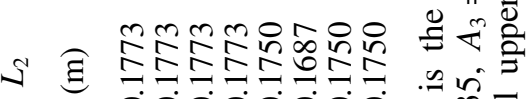

0.700 .090 .00

भी न

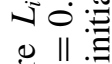

ลิ

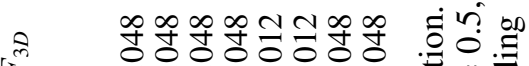

L $\quad 00000000.0$

¿

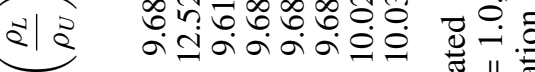

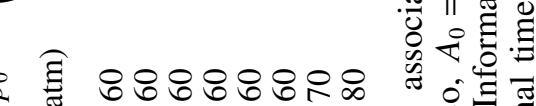
ชิ

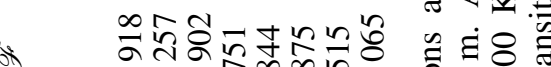
수수의.

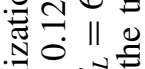

¿ $8888888 \%$ ॠ

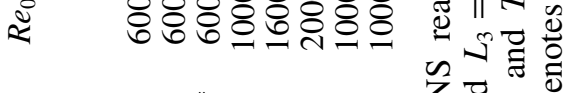

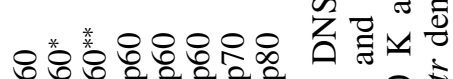
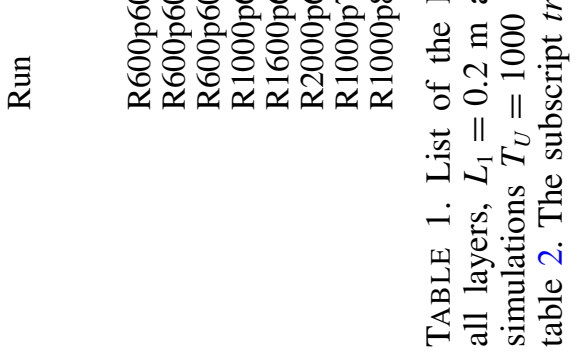
using a fourth-order explicit Runge-Kutta time integration and a sixth-order compact scheme spatial discretization. Time stability was achieved by filtering the conservative variables every five time steps using an eighth-order filter, with the exclusion of the $x_{2}$ boundary since high-order boundary filters were unstable. According to Kennedy \& Carpenter (1994), this filtering only removes spurious information without affecting the physical content of the data as the filter only acts on the shortest wavelengths that can be resolved on the grid and thus does not act as a turbulence model allowing under-resolved computations.

The computations were parallelized using three-dimensional domain decomposition and message passing. The tridiagonal solver for the compact derivative scheme was efficiently parallelized using the method of Muller \& Scheerer (1991).

The grid spacing, $\Delta x$, is uniform and is selected to ensure that the smallest scales relevant to dissipation are resolved and that there is no accumulation of energy at those scales (see §6.2.1). The grid spacings used in various realizations are listed in table 1. For the baseline computation R600p60, the number of grid nodes was first chosen as equal to that of the past binary-species simulations at same $p_{0}$ and $R e_{0}$ values. Then, the number of grid points was sequentially increased to 125,150 and $165 \%$. In each of these simulations a smooth and well-resolved transitional velocity field was obtained. Finally, a grid corresponding to a grid-point increase of $150 \%$ was retained in order to provide a better local and instantaneous representation of the smallest scales. This resolution should not be considered as optimal for such a simulation; indeed, the same grid was successfully used for R1000p60 as well. Scaling the grid with the value of $R e$ is a standardized procedure for fully turbulent flows (Tennekes \& Lumley 1989): the grid points increase as a power law of $R e$, suggested by the relationship $l / \eta_{K} \sim R e^{3 / 4}$ where $l$ is the integral scale and $\eta_{K}$ is the Kolmogorov scale. But such scaling is less obvious for transitional flows where turbulence is not fully developed and the small structures not yet defined. Moreover, given the uncertainty as to whether the resolution is governed by the flow dynamics or by scalar mixing, it is difficult to preliminarily rely on one criterion to determine the resolution; more quantitative assessments are made in $\$ 6.2 .2$. Therefore, a certain amount of experimentation led to the grids listed in table 1 . The domain lengths, $L_{i}$, are the same for all simulations in the $x_{1}$ and $x_{3}$ directions and determined in the $x_{2}$ direction by the relationship $L_{2}=L_{1} *\left(N_{2}-1\right) / N_{1}$ where $N_{i}$ is the number of grid nodes in the direction $i$.

Unlike in Okong'o \& Bellan (2002a), but similar to Okong'o et al. (2002), the EOS was solved through an iterative procedure.

\section{Results}

The simulations are performed here for a mixture of five species selected for their relevance to combustion. The fuel is represented by $\mathrm{C}_{7} \mathrm{H}_{16}$ because it has similar cetane number value to diesel fuel $(\mathrm{CN}$ of $\approx 56$ versus $\approx 50)$; the significance of $\mathrm{CN}$ is that it measures the ignition delay time. Air is here represented by its major species: $\mathrm{N}_{2}$ and $\mathrm{O}_{2}$. Finally, the major products of complete combustion are $\mathrm{CO}_{2}$ and $\mathrm{H}_{2} \mathrm{O}$, both of which complete the set of five species. Listed in table 2 are the initial compositions of the free streams for all simulations. The properties of these five species are listed in table 3. The interaction coefficients necessary to compute $p$ from the EOS for this set of species are provided in table 4; coefficients the value of which is unknown are taken to be null. 


$\begin{array}{lcccccc}\text { Run } & \text { Stream } & Y_{\text {Wat }} & Y_{\text {Car }} & Y_{\text {Oxy }} & Y_{\text {Hep }} & Y_{N} \\ \text { All simulations } & U & 0.01 & 0.035 & 0.2 & 0 & 0.755 \\ \text { (except R600p60*, R600p60**) } & L & 0.01 & 0.035 & 0 & 0.955 & 0 \\ & & & & & & \\ \text { R600p60* } & U & 0.01 & 0.035 & 0.2 & 0 & 0.755 \\ & L & 0 & 0 & 0 & 1 & 0 \\ \text { R600p60** } & U & 0.02 & 0.07 & 0.2 & 0 & 0.71 \\ & L & 0.01 & 0.035 & 0 & 0.955 & 0\end{array}$

TABLE 2. Initial mass fractions of water, carbon dioxide, oxygen, n-heptane and nitrogen in the upper and lower streams.

$\begin{array}{lcccccc}\text { Species } & \begin{array}{c}\text { Species } \\ \text { label }(\alpha)\end{array} & \begin{array}{c}m_{\alpha} \\ \left(\mathrm{kg} \mathrm{kmol}^{-1}\right)\end{array} & \begin{array}{c}T_{c} \\ (\mathrm{~K})\end{array} & \begin{array}{c}p_{c} \\ (\text { bar })\end{array} & \begin{array}{c}v_{c} \\ \left(10^{-3} \mathrm{~m}^{3}\right.\end{array} & \begin{array}{c}\Omega \\ \left.\mathrm{kmol}^{-1}\right)\end{array} \\ \mathrm{H}_{2} \mathrm{O} & 1 & 18.015 & 647.3 & 221 & 57.1 & 0.344 \\ \mathrm{CO}_{2} & 2 & 44.01 & 304.1 & 73.8 & 93.9 & 0.239 \\ \mathrm{O}_{2} & 3 & 32.0 & 154.6 & 50.43 & 73.4 & 0.025 \\ \mathrm{C}_{7} \mathrm{H}_{16} & 4 & 100.2 & 540.2 & 27.4 & 432 & 0.349 \\ \mathrm{~N}_{2} & 5 & 28.013 & 126.26 & 33.4 & 89.8 & 0.039\end{array}$

TABLE 3. Species properties. The species are listed in order of index $\alpha$.

$\begin{array}{lcc}\alpha & \gamma & k^{\prime} \\ \text { Alkane } & \text { Alkane } & 0.0 \\ \text { Alkane } & \mathrm{N}_{2}, \mathrm{O}_{2} & 0.15 \\ \text { Alkane } & \mathrm{CO}_{2} & 0.11 \\ \text { Alkane } & \mathrm{H}_{2} \mathrm{O} & 0.093-0.006 n_{c} \\ \mathrm{CO}_{2} & \mathrm{H}_{2} \mathrm{O} & 0.095 \\ \mathrm{CO}_{2} & \mathrm{~N}_{2}, \mathrm{O}_{2} & -0.017 \\ \mathrm{H}_{2} \mathrm{O} & \mathrm{N}_{2}, \mathrm{O}_{2} & 0.17\end{array}$

TABLE 4. Values of $k^{\prime}$ for species pairs. Here $n_{c}$ is the number of $\mathrm{C}$ atoms in the species.

Complete initial conditions for all computations are listed in table 1; in particular, the values of $\lambda_{1}$ and $\lambda_{3}$ are $\lambda_{1} / \delta_{\omega, 0}=7.29$ and $\lambda_{3}=0.6 \lambda_{1}$ as in Moser \& Rogers (1991). Each realization reached a transitional state denoted by the subscript $t r$ and two important quantities, namely the non-dimensional transitional time $t_{t r}^{*}$ (time at which the one-dimensional fluctuation-based energy spectra become smooth, except for the forcing frequency) where $t^{*}=t \Delta U_{0} / \delta_{\omega, 0}$, and the momentum-thickness-based Reynolds number defined below in (6.1), also appear in table 1.

Because simulations with an analytical perturbation have not been performed in the past and thus we are unfamiliar with how the layer grows under these conditions, we first inquire in $\S 6.1$ about the evolution of the global quantities up to transition. Then, in $\S 6.2$ we focus on the mixing layer characteristics at transition encompassing the spectra in $\S 6.2 .1$, the transport properties in $\$ 6.2 .2$, the thermodynamic characteristics of the mixture in $\S 6.2 .3$ where we also discuss the equivalence ratio distribution. Finally, since dissipation is a gradient-induced process which is due to transport properties, we investigate in $\S 6.3$ the irreversible entropy production (which is the dissipation). 
All figures showing spatial distributions have had the range of values cropped at both the small-value and large-value end so as to enable distilling the essence of physics from the significant values. Thus, the points having a value in the range outside the smallest or the largest value shown in the legend of each figure are included in the corresponding end range.

\subsection{Evolution of the global quantities}

To understand the global evolution of the mixing layer perturbed with the analytical perturbation developed by this study and detailed in appendix B.2, we compute and examine here the momentum thickness

$$
\delta_{m}=\frac{\int_{x_{2}, \min }^{x_{2}, \max }\left[\left\langle\rho u_{1}\right\rangle_{x_{2}, \max }-\left\langle\rho u_{1}\right\rangle\right]\left[\left\langle\rho u_{1}\right\rangle-\left\langle\rho u_{1}\right\rangle_{x_{2}, \min }\right] \mathrm{d} x_{2}}{\left(\left\langle\rho u_{1}\right\rangle_{x_{2}, \max }-\left\langle\rho u_{1}\right\rangle_{x_{2}, \min }\right)^{2}},
$$

which is a measure of layer growth, where $x_{2, \min }=-L_{2} / 2.5, x_{2, \max }=x_{2, \min }+L_{2}$ and \langle\rangle symbolizes averages over homogeneous $\left(x_{1}, x_{3}\right)$ planes. Figure 1 illustrates $\delta_{m} / \delta_{\omega, 0}$ as a function of $t^{*}$ for several of the runs listed in table 1. Concurrently, we also calculate and analyse the domain-averaged positive spanwise vorticity $\left\langle\left\langle\omega_{3}^{+}\right\rangle\right\rangle \delta_{\omega, 0} / \Delta U_{0}$ which is an indication of small-scale formation in the layer considering that all initial spanwise vorticity is negative, and the domain-averaged enstrophy $\left\langle\left\langle\omega_{i} \omega_{i}\right\rangle\right\rangle\left(\delta_{\omega, 0} / \Delta U_{0}\right)^{2} \delta_{m} / \delta_{\omega, 0}$ which is a manifestation of the stretching and twisting aspects of turbulence where $\langle\langle\rangle\rangle$ denotes entire domain averaging.

Clearly, the analytical perturbation has the advantage of accelerating the attainment of a state close to self-similarity where the layer grows at a constant rate, here determined by the slope of $\delta_{m} / \delta_{\omega, 0}$. This constant rate abates once the layer approaches the transitional state identified by the $t^{*}$ value at which the spectra are smooth (see $\S 6.2 .1)$. Increasing $R e_{0}$ from 600 to 1000 at the same $p_{0}$ and the same perturbation amplitudes $F_{3 D}$ and $F_{2 D}$ results in a slightly larger entrainment and thus growth after $t^{*} \approx 25$, however, with increasing entrainment a larger portion of the heavier lower stream is present in the layer, which damps its growth. Indeed, $t^{*} \approx 60$, when the growth of R1000p60 becomes smaller than that of R600p60, corresponds to a slight decrease in small-scale production initiated close to $t^{*} \approx 50$, at which time the stretching and twisting aspects of the layer have culminated. The larger $R e_{0}$ allows the layer to recover from the temporary abatement of small-scale production but this recovery does not exceed the peak occurring at $t^{*} \approx 50$, unlike for R600p60. Increasing $p_{0}$ to 80 atm at otherwise same initial conditions does not have a visible impact on $\delta_{m} / \delta_{\omega, 0}$, but due to the heavier fluid at the larger pressure the vortical aspects of the layer represented by both $\left\langle\left\langle\omega_{3}^{+}\right\rangle\right\rangle \delta_{\omega, 0} / \Delta U_{0}$ and $\left\langle\left\langle\omega_{i} \omega_{i}\right\rangle\right\rangle\left(\delta_{\omega, 0} / \Delta U_{0}\right)^{2}$ are diminished.

Computations with $R e_{0} \geqslant 1600$ required smaller values of $F_{3 D}$ and $F_{2 D}$ (see table 1) because otherwise the computation showed lack of convergence at very early stages. The smaller perturbations led to a delayed growth, but once the layer expanded, as expected, its rate of growth exceeded the growth rate of the lower value $R e_{0}$ layers, as manifested by the slope of $\delta_{m} / \delta_{\omega, 0}$. Consistent with the larger $R e_{0}$ value, the vortical features of R1600p60 and R2000p60 eventually surpass those of R1000p60, and past $R e_{0}=1000$ the peak of $\left\langle\left\langle\omega_{i} \omega_{i}\right\rangle\right\rangle\left(\delta_{\omega, 0} / \Delta U_{0}\right)^{2}$ is approximately proportional in magnitude to the $R e_{0}$ value.

As an indication of turbulence level achieved, the value of $\operatorname{Re}_{m} \equiv \operatorname{Re}_{0} \delta_{m} / \delta_{\omega, 0}$ corresponding to the $\delta_{m} / \delta_{\omega, 0}$ value at transition, is provided in table 1 . 

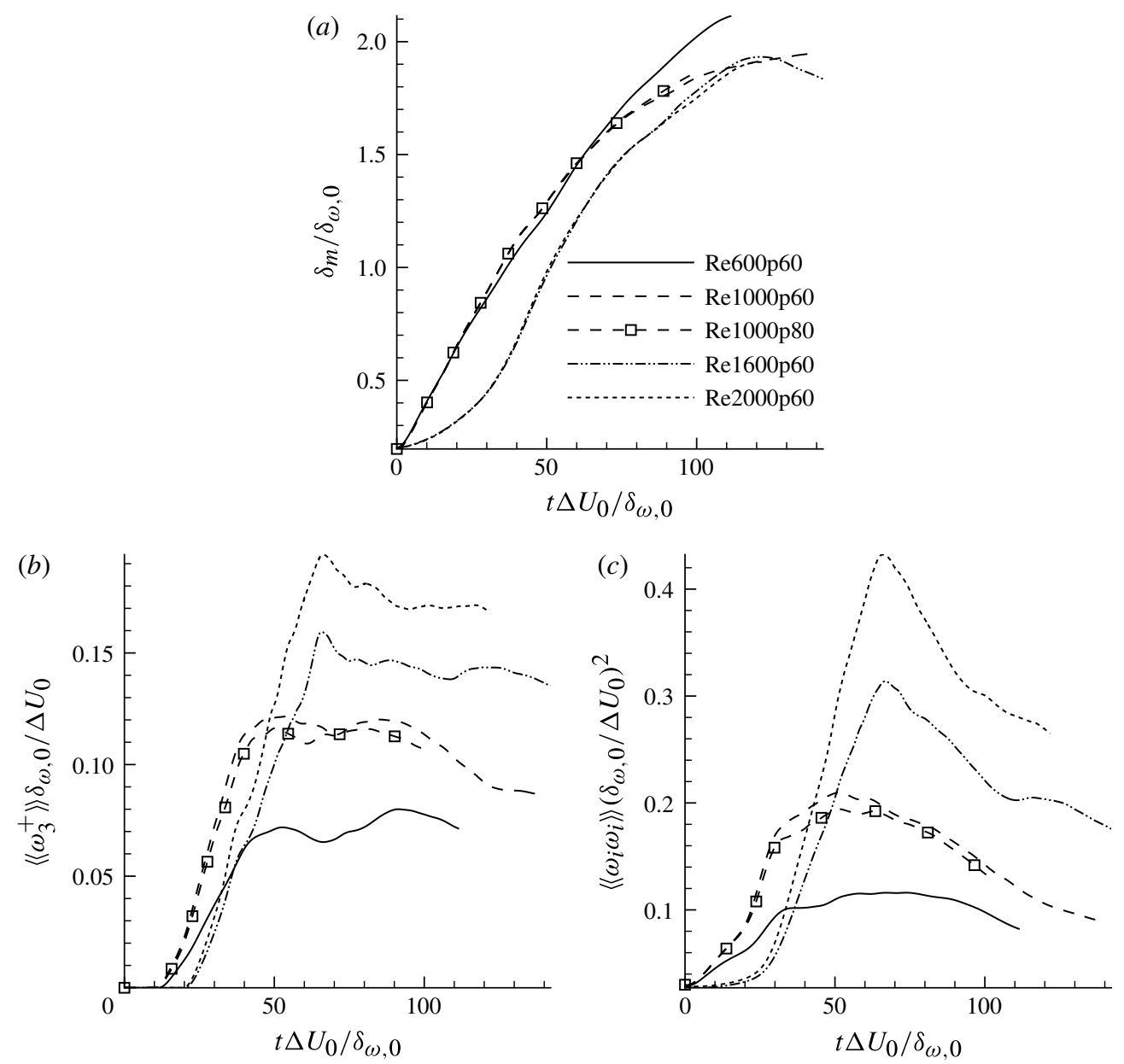

FIGURE 1. Time-wise evolution of integral quantities: $(a) \delta_{m} / \delta_{\omega, 0} ;(b)\left\langle\left\langle\omega_{3}^{+}\right\rangle\right\rangle \delta_{\omega, 0} / \Delta U_{0}$; and (c) $\left\langle\left\langle\omega_{i} \omega_{i}\right\rangle\right\rangle\left(\delta_{\omega, 0} / \Delta U_{0}\right)^{2}$ for some of the simulations listed in table 1 .

\subsection{Mixing layer characteristics at transition}

\subsubsection{One-dimensional spectra}

Figure 2 displays the spanwise and streamwise spectra, $E(k)$, of the streamwise velocity $u_{1}$, and of both scalars $Y_{H e p}$ and $T$ at $t_{t r}^{*}$, with the $k^{-5 / 3}$ curve for comparison, where $k$ denotes the wavenumber. Because the relationship $\eta_{B}=\eta_{K} S c^{-0.5}$ assumes the existence of a single $S c$ for the mixture which is not necessarily the case (see $\S 6.2 .2$ ), examining the resolution for the mass fractions is just as important as assessing the resolution of the dynamic scales, as the former cannot indubitably be inferred from the latter. The relationship $\eta_{\theta}=\eta_{K} \mathrm{Pr}^{-0.5}$ prompts us to also check the resolution of the thermal scales. The spectra of all quantities show the smoothness associated with turbulent characteristics (except for the small peak at the perturbation frequency) and the excellent resolution, with no energy accumulation at the smallest scales. As expected, the simulations at the larger $R e_{0}$ values have spectra which extend over a wider range of scales and have more energy in the smallest scales than at the smaller $R e_{0}$ values. Increasing $p_{0}$ from 60 to 80 atm has no visible influence on the spectra. 
(a)

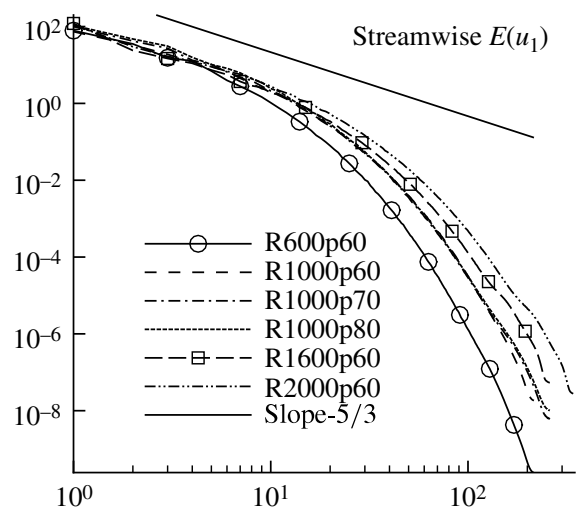

$(c)$

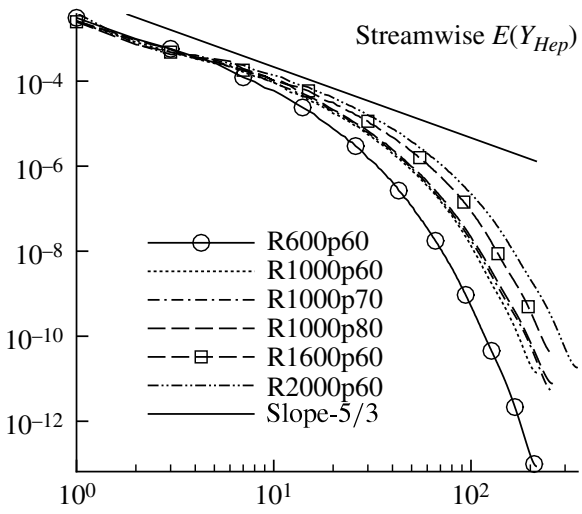

$(e)$

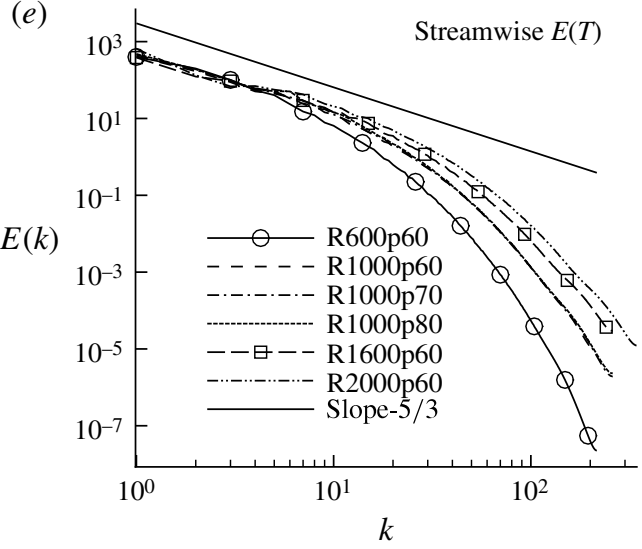

(b)

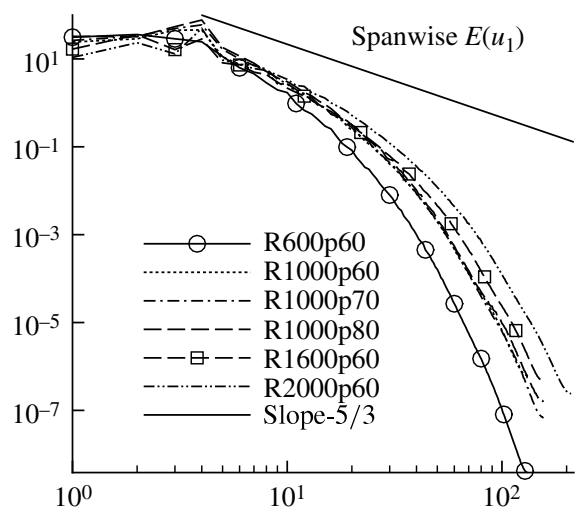

(d)

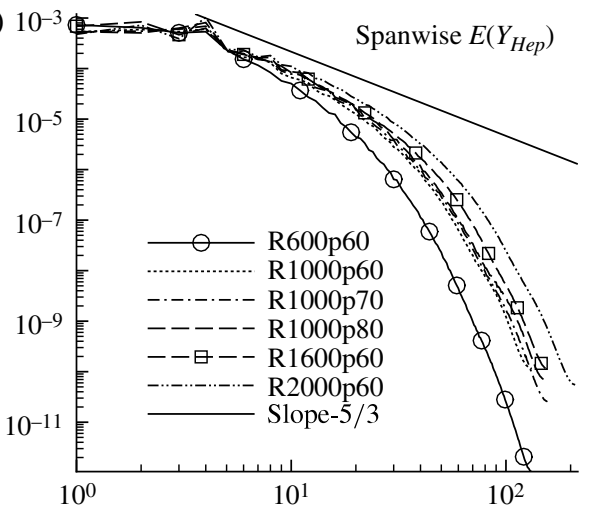

$(f)$

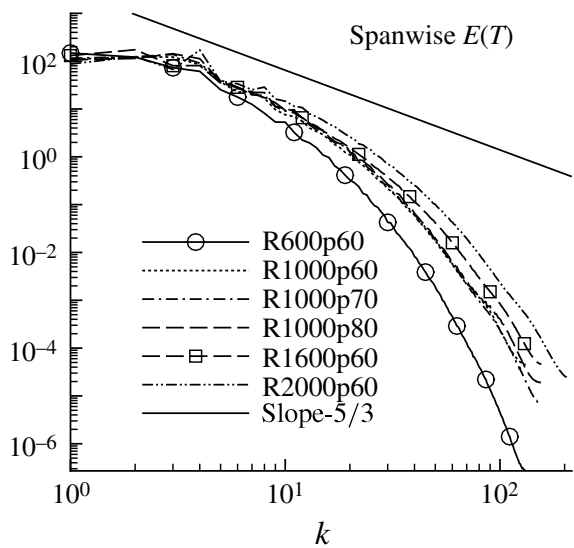

FIGURE 2. One-dimensional spectra at $t_{t r}^{*}$ for several simulations: streamwise spectra of $(a)$ $u_{1}$, (c) $Y_{H e p},(e) T$; spanwise spectra of $(b) u_{1},(d) Y_{H e p},(f) T$.

\subsubsection{Transport properties features}

In a turbulent flow, mixing occurs at many scales, the smallest one in the continuum range being $\eta_{K}$. But mixing also occurs at scales smaller than $\eta_{K}$; this is molecular mixing due to diffusion. Molecular mixing affects the continuum range of scales and strongly influences phenomena such as chemical reactions since species can only react 
if they are brought together. The characteristic scale over which molecular diffusion occurs is thus of paramount importance in understanding mixing. The Schmidt number, which measures the importance of momentum transfer with respect to mass transfer, can provide an estimate of this characteristic length, but the caveat is that $S c$ is only well defined when there is a single mass-diffusion coefficient, i.e. for a binaryspecies system. For a mixture of several species for which a complete diffusion matrix applies, in principle, a Schmidt number can be defined in conjunction with each massdiffusion coefficient of the matrix. This definition is not useful because it does not provide information about a single diffusion length scale associated with each species. Moreover, for supercritical flows where Soret effects may be important, the species diffusivity cannot entirely represent the species mass-diffusion scale. Species diffusion is very important not only on its own, but also because the heat flux contains a term expressing the transport of enthalpy with the species fluxes, and thus the definition of an effective Schmidt number for a species, $S c_{\alpha, \text { eff }}$, has direct bearing on the definition of an effective Prandtl number, $P r_{\text {eff }}$, which measures the importance of momentum transfer with respect to heat transfer and can provide a characteristic scale for the latter.

6.2.2.1. Formalism for deriving $S c_{\alpha, \text { eff }}$. To define $S c_{\alpha, \text { eff }}$, consider (2.4) here rewritten to emphasize the diffusion flux

$$
\frac{\partial\left(\rho Y_{\alpha}\right)}{\partial t}+\frac{\partial\left(\rho u_{j} Y_{\alpha}\right)}{\partial x_{j}}=\frac{\partial}{\partial x_{j}}\left(\rho Y_{\alpha} \frac{D_{T, \alpha}}{T} \frac{\partial T}{\partial x_{j}}+\rho Y_{\alpha} \frac{D_{p, \alpha}}{p} \frac{\partial p}{\partial x_{j}}+\rho \sum_{\beta=1}^{N-1} \mathfrak{D}_{\alpha \beta} \frac{\partial Y_{\beta}}{\partial x_{j}}\right),
$$

where

$$
\mathfrak{D}_{\alpha \beta}=D_{\alpha \beta}^{\prime} \frac{m_{\alpha}}{m_{\beta}} .
$$

For a mixture of five species, one may generically write

$$
Y_{1}=f_{1}(\boldsymbol{x}, t), \quad Y_{2}=f_{2}(\boldsymbol{x}, t), \quad Y_{3}=f_{3}(\boldsymbol{x}, t), \quad Y_{4}=f_{4}(\boldsymbol{x}, t)
$$

(with the consequence that $Y_{5}=1-f_{1}(\boldsymbol{x}, t)-f_{2}(\boldsymbol{x}, t)-f_{3}(\boldsymbol{x}, t)-f_{4}(\boldsymbol{x}, t)$ ) and similarly

$$
T=f_{T}(\boldsymbol{x}, t) \quad \text { and } \quad p=f_{p}(\boldsymbol{x}, t) .
$$

Under the assumption that

$$
Y_{\alpha}=g\left(f_{\beta}(\boldsymbol{x}, t)\right), \quad T=l\left(f_{\alpha}(\boldsymbol{x}, t)\right), \quad p=r\left(f_{\alpha}(\boldsymbol{x}, t)\right)
$$

where $\beta \neq \alpha$ and where $g, l$ and $r$ are composite functions, one can write

$$
\frac{\partial Y_{\alpha}}{\partial x_{i}}=\frac{\partial g\left(f_{\beta}(\boldsymbol{x}, t)\right)}{\partial x_{i}}=\frac{\delta g\left(f_{\beta}(\boldsymbol{x}, t)\right)}{\delta f_{\beta}(\boldsymbol{x}, t)} \frac{\partial f_{\beta}(\boldsymbol{x}, t)}{\partial x_{i}}
$$

and

$$
\frac{\partial T}{\partial x_{i}}=\frac{\partial l\left(f_{\alpha}(\boldsymbol{x}, t)\right)}{\partial x_{i}}=\frac{\delta l\left(f_{\alpha}(\boldsymbol{x}, t)\right)}{\delta f_{\alpha}(\boldsymbol{x}, t)} \frac{\partial f_{\alpha}(\boldsymbol{x}, t)}{\partial x_{i}}
$$

with a similar expression for $p$ where $\delta$ represents a functional derivative. The assumption of (6.6) is reasonable in the mixing layer region since gradients are generally non-null; furthermore $\sum_{\beta=1}^{N} Y_{\beta}=1$ and $\sum_{\beta=1}^{N} \boldsymbol{J}_{\beta}=0$ showing that all species are related, and $T$ and $p$ are coupled to the species through the governing equations. 
Equations (6.6) become increasingly valid as the gradients are larger. Then, one can write

$$
\begin{aligned}
\frac{\partial\left(\rho Y_{\alpha}\right)}{\partial t}+\frac{\partial\left(\rho u_{j} Y_{\alpha}\right)}{\partial x_{j}}= & \frac{\partial}{\partial x_{j}}\left(\rho Y_{\alpha} \frac{D_{T, \alpha}}{T} \frac{\delta T}{\delta Y_{\alpha}} \frac{\partial Y_{\alpha}}{\partial x_{j}}+\rho Y_{\alpha} \frac{D_{p, \alpha}}{p} \frac{\delta p}{\delta Y_{\alpha}} \frac{\partial Y_{\alpha}}{\partial x_{j}}\right. \\
& \left.+\rho \sum_{\beta=1}^{N-1} \mathfrak{D}_{\alpha \beta} \frac{\delta Y_{\beta}}{\delta Y_{\alpha}} \frac{\partial Y_{\alpha}}{\partial x_{j}}\right) \\
\frac{\partial\left(\rho Y_{\alpha}\right)}{\partial t}+\frac{\partial\left(\rho u_{j} Y_{\alpha}\right)}{\partial x_{j}}= & \frac{\partial}{\partial x_{j}}\left[\rho \left(Y_{\alpha} \frac{D_{T, \alpha}}{T} \frac{\delta T}{\delta Y_{\alpha}}+Y_{\alpha} \frac{D_{p, \alpha}}{p} \frac{\delta p}{\delta Y_{\alpha}}\right.\right. \\
& \left.\left.+\sum_{\beta=1}^{N-1} \mathfrak{D}_{\alpha \beta} \frac{\delta Y_{\beta}}{\delta Y_{\alpha}}\right) \frac{\partial Y_{\alpha}}{\partial x_{j}}\right] \\
\frac{\partial\left(\rho Y_{\alpha}\right)}{\partial t}+\frac{\partial\left(\rho u_{j} Y_{\alpha}\right)}{\partial x_{j}}= & \frac{\partial}{\partial x_{j}}\left[\rho \check{D}_{\alpha} \frac{\partial Y_{\alpha}}{\partial x_{j}}\right]
\end{aligned}
$$

where

$$
\check{D}_{\alpha} \equiv\left(Y_{\alpha} \frac{D_{T, \alpha}}{T} \frac{\delta T}{\delta Y_{\alpha}}+Y_{\alpha} \frac{D_{p, \alpha}}{p} \frac{\delta p}{\delta Y_{\alpha}}+\sum_{\beta=1}^{N-1} \mathfrak{D}_{\alpha \beta} \frac{\delta Y_{\beta}}{\delta Y_{\alpha}}\right)
$$

represents a single species diffusion coefficient accounting for the entire flux matrix pertaining to species $\alpha$. One can thus define an effective species Schmidt number as

$$
S c_{\alpha, e f f}=\frac{\mu}{\rho \check{D}_{\alpha}}
$$

6.2.2.2. Formalism for deriving $P r_{\text {eff. }}$. Similar to the above, equation (2.3) can be written as

$$
\frac{\partial\left(\rho e_{t}\right)}{\partial t}+\frac{\partial\left[\left(\rho e_{t}+p\right) u_{j}-u_{i} \sigma_{i j}\right]}{\partial x_{j}}=\frac{\partial}{\partial x_{j}}\left(\lambda \frac{\partial T}{\partial x_{j}}-\sum_{\alpha=1}^{N-1} J_{\alpha, j} A_{\alpha}\right)
$$

where

$$
A_{\alpha}=\left[\left(\frac{h_{\alpha}}{m_{\alpha}}-\frac{h_{N}}{m_{N}}\right)-R_{u} T\left(\frac{\bar{\alpha}_{T, \alpha}^{b}}{m_{\alpha}}-\frac{\bar{\alpha}_{T, N}^{b}}{m_{N}}\right)\right],
$$

and further manipulations similar to those for (6.9)-(6.11) lead to

$$
\frac{\partial\left(\rho e_{t}\right)}{\partial t}+\frac{\partial\left[\left(\rho e_{t}+p\right) u_{j}-u_{i} \sigma_{i j}\right]}{\partial x_{j}}=\frac{\partial}{\partial x_{j}}\left[\Lambda \frac{\partial T}{\partial x_{j}}\right],
$$

where

$$
\begin{aligned}
\Lambda & \equiv\left\{\lambda+\rho \sum_{\alpha=1}^{N-1} A_{\alpha}\left[Y_{\alpha}\left(\frac{D_{T, \alpha}}{T}+\frac{D_{p, \alpha}}{p} \frac{\delta p}{\delta T}\right)+\sum_{\beta=1}^{N-1} \mathfrak{D}_{\alpha \beta} \frac{\delta Y_{\beta}}{\delta T}\right]\right\} \\
& =\left(\lambda+\rho \sum_{\alpha=1}^{N-1} A_{\alpha} \breve{D}_{\alpha} \frac{\delta Y_{\alpha}}{\delta T}\right)
\end{aligned}
$$


which represents a local effective conductivity accounting for the entire heat flux pertaining to all species. Therefore, $\Lambda$ can serve to define an effective Prandtl number

$$
P r_{e f f}=\frac{C_{p} \mu}{\Lambda} .
$$

6.2.2.3. Modelling of $S c_{\alpha, \text { eff }}$ and $P r_{\text {eff }}$. The definitions of (6.12) and (6.17) are convenient but because $\delta T / \delta Y_{\alpha}, \delta Y_{\beta} / \delta Y_{\alpha}, \delta Y_{\beta} / \delta T, \delta p / \delta Y_{\alpha}$ and $\delta p / \delta T$ are usually not available or not easily calculable, the $S c_{\alpha, \text { eff }}$ and $P r_{\text {eff }}$ definitions can only be useful if a model for computing $\check{D}_{\alpha}$ and $\Lambda$ is proposed. The real test of the model accuracy is the recovery of the fluxes computed from the database according to the definitions (2.6) and (2.7); this is the same methodology as a priori modelling used, for example, in the context of subgrid-scale models (e.g. Selle et al. 2007).

To model $\delta T / \delta Y_{\alpha}, \delta Y_{\beta} / \delta Y_{\alpha}, \delta Y_{\beta} / \delta T, \delta p / \delta Y_{\alpha}$ and $\delta p / \delta T$ in (6.12) and (6.17), we define the following coefficients

$$
\begin{aligned}
C_{\beta \alpha} & \equiv \frac{\delta Y_{\beta}}{\delta Y_{\alpha}}, \quad C_{\beta T} \equiv \frac{\delta Y_{\beta}}{\delta T}, \\
C_{T \alpha} & \equiv \frac{\delta T}{\delta Y_{\alpha}}, \\
C_{p \alpha} & \equiv \frac{\delta p}{\delta Y_{\alpha}}, \quad C_{p T} \equiv \frac{\delta p}{\delta T},
\end{aligned}
$$

that also exactly satisfy

$$
\begin{aligned}
C_{\beta \alpha} \frac{\partial Y_{\alpha}}{\partial x_{i}} & =\frac{\partial Y_{\beta}}{\partial x_{i}}, \quad C_{\beta T} \frac{\partial T}{\partial x_{i}}=\frac{\partial Y_{\beta}}{\partial x_{i}}, \\
C_{T \alpha} \frac{\partial Y_{\alpha}}{\partial x_{i}} & =\frac{\partial T}{\partial x_{i}}, \\
C_{p \alpha} \frac{\partial Y_{\alpha}}{\partial x_{i}} & =\frac{\partial p}{\partial x_{i}}, \quad C_{p T} \frac{\partial T}{\partial x_{i}}=\frac{\partial p}{\partial x_{i}},
\end{aligned}
$$

where $\alpha \in[1, N-1]$ and $\beta \in[1, N-1]$. Because there is only one $C_{\beta \alpha}$, yet (6.22) shows that in principle it could be computed using derivatives in any of the $i$ directions, the expectation is that the variation according to direction should be small and only depend on numerical aspects if the functional dependency assumption of (6.6) is satisfied. To eliminate potential directional variations, we compute these coefficients using a model employing contracted products that is insensitive to such directional variations

$$
\begin{aligned}
C_{\beta \alpha} & =\left(\frac{\partial Y_{\beta}}{\partial x_{i}} \frac{\partial Y_{\alpha}}{\partial x_{i}}\right) /\left(\frac{\partial Y_{\alpha}}{\partial x_{i}} \frac{\partial Y_{\alpha}}{\partial x_{i}}\right), \quad C_{\beta T}=\left(\frac{\partial Y_{\beta}}{\partial x_{i}} \frac{\partial T}{\partial x_{i}}\right) /\left(\frac{\partial T}{\partial x_{i}} \frac{\partial T}{\partial x_{i}}\right), \\
C_{T \alpha} & =\left(\frac{\partial T}{\partial x_{i}} \frac{\partial Y_{\alpha}}{\partial x_{i}}\right) /\left(\frac{\partial Y_{\alpha}}{\partial x_{i}} \frac{\partial Y_{\alpha}}{\partial x_{i}}\right), \\
C_{p \alpha} & =\left(\frac{\partial p}{\partial x_{i}} \frac{\partial Y_{\alpha}}{\partial x_{i}}\right) /\left(\frac{\partial Y_{\alpha}}{\partial x_{i}} \frac{\partial Y_{\alpha}}{\partial x_{i}}\right), \quad C_{p T}=\left(\frac{\partial p}{\partial x_{i}} \frac{\partial T}{\partial x_{i}}\right) /\left(\frac{\partial T}{\partial x_{i}} \frac{\partial T}{\partial x_{i}}\right) .
\end{aligned}
$$

Not only does the model, (6.25)-(6.27), render the coefficients independent of directional variation, but it also avoids potential singularities occurring when there are very small gradients (the limit of applicability of (6.6)). Only some of the relationships 
(6.25)-(6.27) are independent, and 5 coefficients out of the total 25 must be computed (without counting coefficients $C_{\alpha \alpha}=1$ ).

We label as 'reformulated' the formalism in which the coefficients $C_{\beta \alpha}$, etc. are computed according to (6.22) and (6.24), with the result that in principle there are three quantities $\check{D}_{\alpha, i}$ which could vary according to direction. In contrast, we denote as 'modelled' the formalism in which the coefficients $C_{\beta \alpha}$, etc. are computed according to (6.25)-(6.27) and which leads to a single quantity $\check{D}_{\alpha}$.

The model, (6.25)-(6.27), is assessed through comparisons of modelled fluxes (defined as the terms in the square brackets in (6.11) and (6.16) multiplied by $(-1)$, in combination with (6.25) and (6.27) to compute the coefficients) and exact fluxes $J_{\text {Hep } 2}, J_{\text {Wat } 2}$ and $q_{2}$ (i.e. equation (2.6) and (2.7)), where the choice of the $x_{2}$ coordinate is motivated by the fact that diffusion is predominantly cross-stream. For $\mathrm{C}_{7} \mathrm{H}_{16}$ and $\mathrm{O}_{2}$, diffusion is generally one way in the $x_{2}$ direction whereas for $\mathrm{CO}_{2}$ and $\mathrm{H}_{2} \mathrm{O}$ diffusion changes according to the location in the $x_{2}$ direction since these latter two species are initially present in both streams for all simulations except R600p60*. (If the initial mass fraction of $\mathrm{CO}_{2}$ and $\mathrm{H}_{2} \mathrm{O}$ is the same in the two streams but $T_{U} \neq T_{L}$, diffusion is initiated by the Soret effect and by the contribution in $\boldsymbol{J}_{\text {Wat }}$ and $\boldsymbol{J}_{\text {Car }}$ of the diffusion coefficients describing the diffusional interaction of $\mathrm{CO}_{2}$ and $\mathrm{H}_{2} \mathrm{O}$ with the other species.) Results are depicted in figures 3-5 for R1000p60, R1000p80 and R2000p60 at the respective $t_{t r}^{*}$. The assessment is performed from examination of conditional averages computed over the entire mixing layer $0.01 \leqslant Y_{\text {Hep }} \leqslant 0.945$, and from inspection of joint probability density functions (p.d.f.s) representing a more demanding requirement than conditional averages because they are pointwise functions. The results show that independent of the simulation, the conditional averages for $J_{\text {Hep } 2}$ and $q_{2}$ are excellently modelled and indistinguishable from the perfect $45^{\circ}$ line, while $J_{\text {Wat } 2}$ is very well reproduced with only very minor deviations from the exact values. The joint p.d.f. for $\mathrm{C}_{7} \mathrm{H}_{16}$ also shows a very high-fidelity duplication by the model of the actual flux. For $\mathrm{H}_{2} \mathrm{O}$ the model shows the preferential direction of the exact flux, but it appears that the coefficients $C_{\beta \alpha}$ as defined by (6.19) are not equal for all directions, which creates a moderate discrepancy between model and actual values. For example, visualizations of $C_{\text {Hep,Wat }}$ computed according to (6.22) in the $x_{i}$ directions are illustrated in figure 6 (where the coefficient is called $C_{41}$ ) showing that the coefficient values are similar but not the same. This indicates that the assumption of (6.6) is generally but not always verified for $\mathrm{H}_{2} \mathrm{O}$, this being attributed to the fact that $Y_{W a t}$ is initially uniform in the entire domain for R1000p60, R1000p80 and R2000p60 and thus diffusion is not vigorous; this fact is indicated by the much smaller values of $J_{\text {Wat } 2}$ than $J_{\text {Hep } 2}$ (a factor of $10^{2}$ for R1000p60, R1000p80 and R2000p60). Obviously, the model is more prone to being in error trying to reproduce smaller rather than larger flux values since it is based on the premise of substantial gradients. Regarding $q_{2}$, the joint p.d.f. shows that the model reproduces considerably well the actual value for all of the simulations.

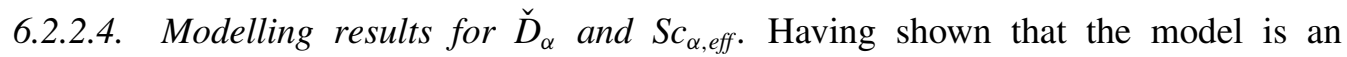
excellent to a very good representation of the exact flux, we are now in a position to examine $\check{D}_{\alpha}$ and thus, $S c_{\alpha, \text { eff }}$. Results for $\check{D}_{\text {Hep }}, S c_{\text {Hep eff }}, \check{D}_{\text {Wat }}$ and $S c_{\text {Wat eff }}$ in the $x_{3} / L_{3}=1 / 16$ plane are illustrated in figure 7 for R1000p60, R1000p80 and R2000p60 at the respective $t_{t r}^{*}$. The $\mathrm{O}_{2}\left(\right.$ and $\left.\mathrm{N}_{2}\right)$ results are similar to those of $\mathrm{C}_{7} \mathrm{H}_{16}$, and those of $\mathrm{CO}_{2}$ are similar to those of $\mathrm{H}_{2} \mathrm{O}$. The shown $\check{D}_{\alpha}$ values are those unscaled by $\mathscr{F}$. $\check{D}_{\text {Hep }}>0$ (figure $7 a-c$ ), varying by approximately a factor of six within the layer, 

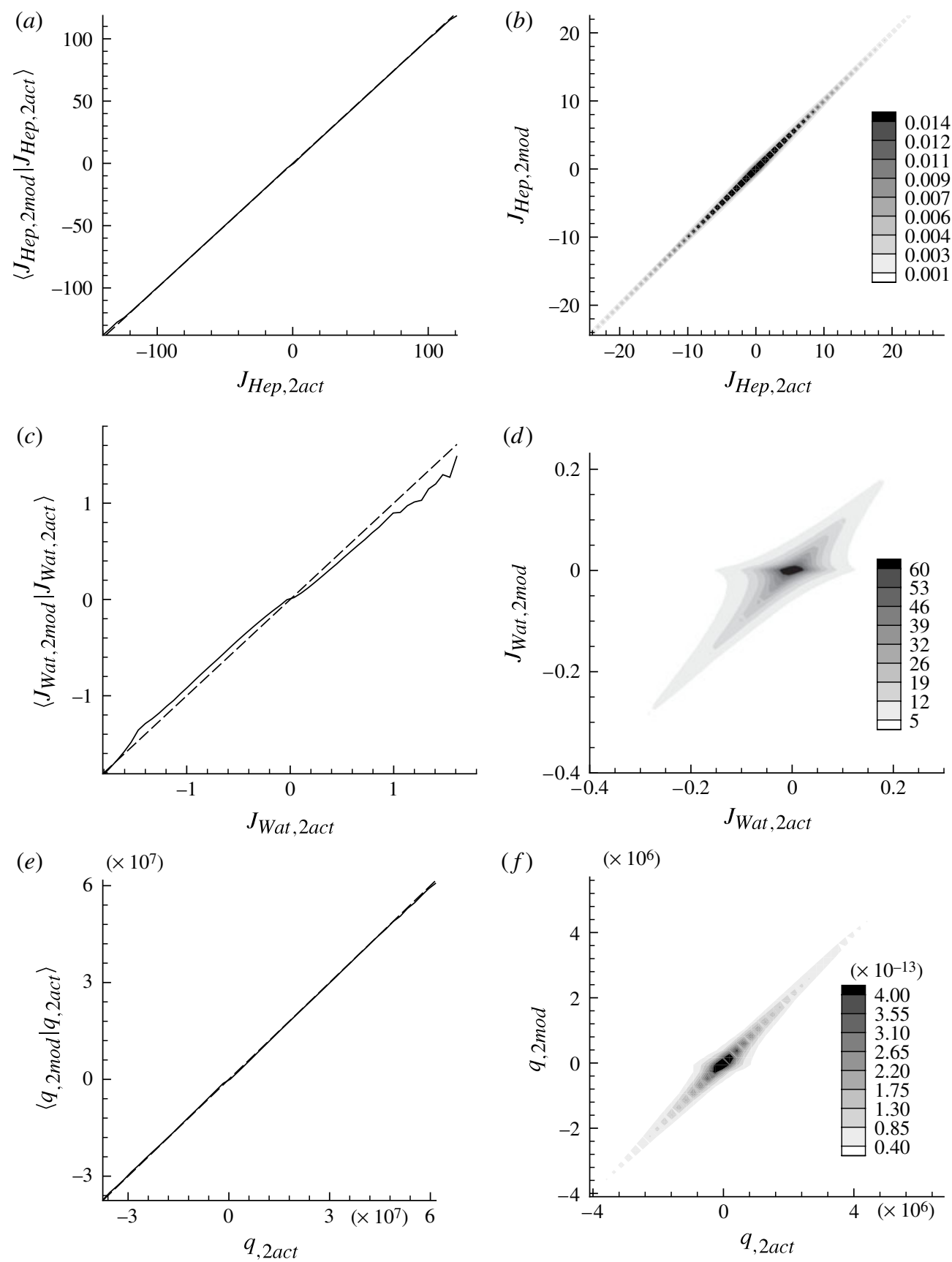

FIGURE 3. Conditional averages of the modelled with respect to the exact (solid line) for $J_{H e p 2}, J_{W a t 2}$ and $q_{2}(a, c, e)$ and joint p.d.f. of the modelled and exact $Y_{H e p}, J_{W a t 2}$ and $q_{2}(b, d, f)$, both computed into the mixing layer $\left(0.01 \leqslant Y_{H e p} \leqslant 0.945\right)$ at $t_{t r}^{*}$ for R1000p60. For the conditional averages the dashed line represents the $45^{\circ}$ slope indicating perfect agreement. The joint p.d.f. is evaluated in the range of \pm 3 times the standard deviation from the mean value. 

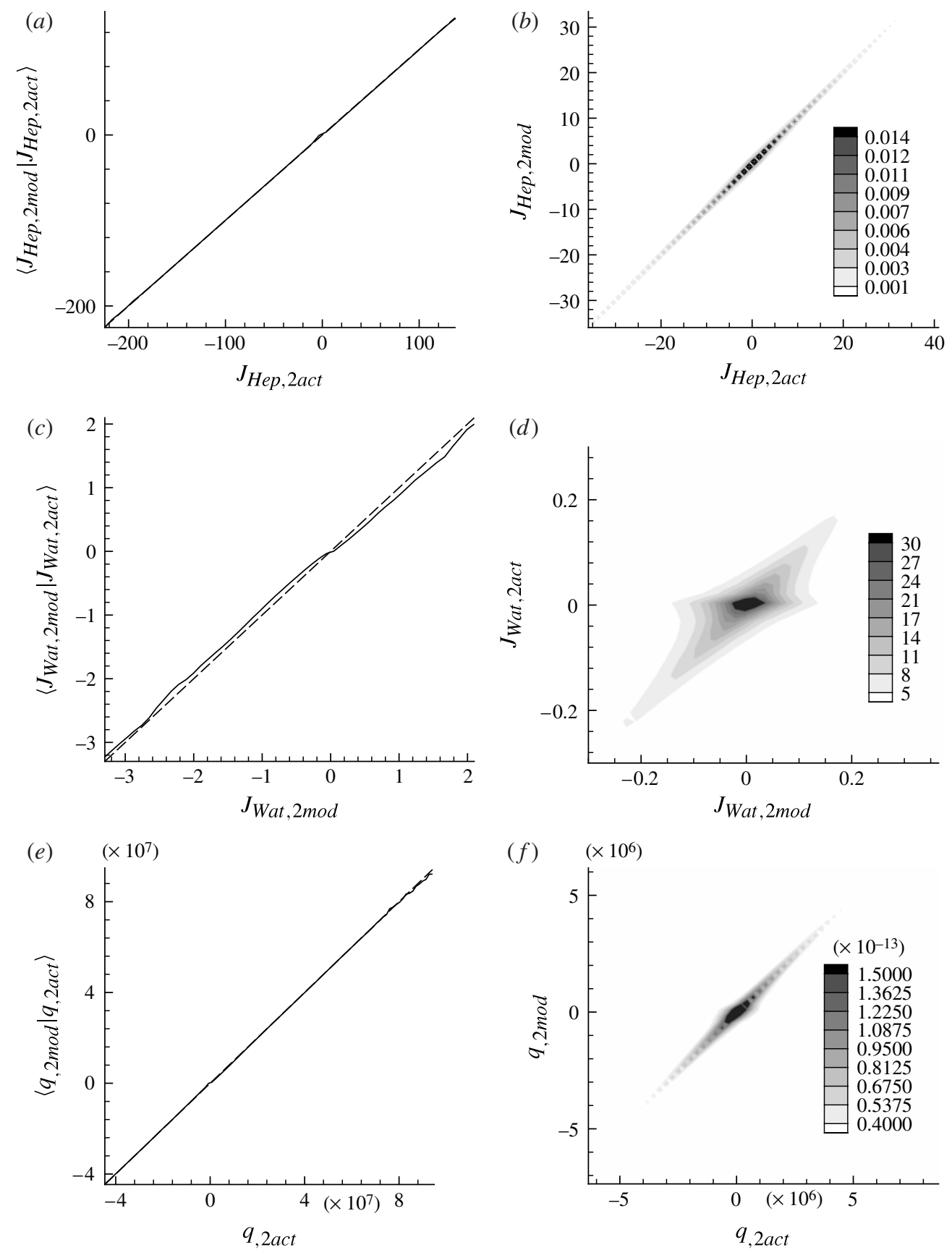

FIGURE 4. Conditional averages of the modelled with respect to the exact (solid line) for $J_{\text {Hep } 2}, J_{\text {Wat } 2}$ and $q_{2}(a, c, e)$ and joint p.d.f. of the modelled and exact $Y_{\text {Hep }}, J_{\text {Wat } 2}$ and $q_{2}(b, d, f)$, both computed into the mixing layer $\left(0.01 \leqslant Y_{H e p} \leqslant 0.945\right)$ at $t_{t r}^{*}$ for R1000p80. For the conditional averages the dashed line represents the $45^{\circ}$ slope indicating perfect agreement. The joint p.d.f. is evaluated in the range of \pm 3 times the standard deviation from the mean value. 

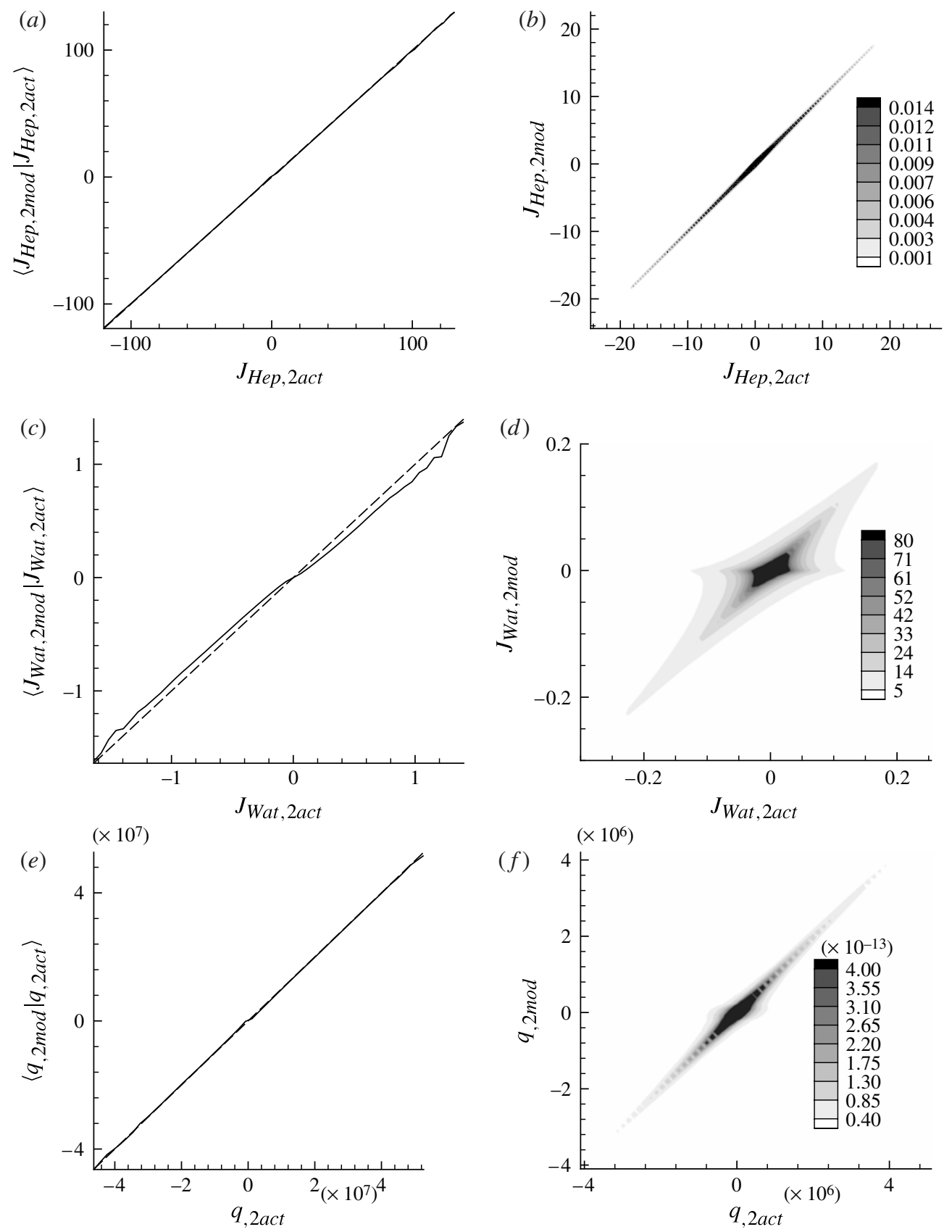

FIGURE 5. Conditional averages of the modelled with respect to the exact (solid line) for $J_{H e p 2}, J_{W a t 2}$ and $q_{2}(a, c, e)$ and joint p.d.f. of the modelled and exact $Y_{H e p}, J_{\text {Wat } 2}$ and $q_{2}(b, d, f)$, both computed into the mixing layer $\left(0.01 \leqslant Y_{H e p} \leqslant 0.945\right)$ at $t_{t r}^{*}$ for R2000p60. For the conditional averages the dashed line represents the $45^{\circ}$ slope indicating perfect agreement. The joint p.d.f. is evaluated in the range of \pm 3 times the standard deviation from the mean value. 

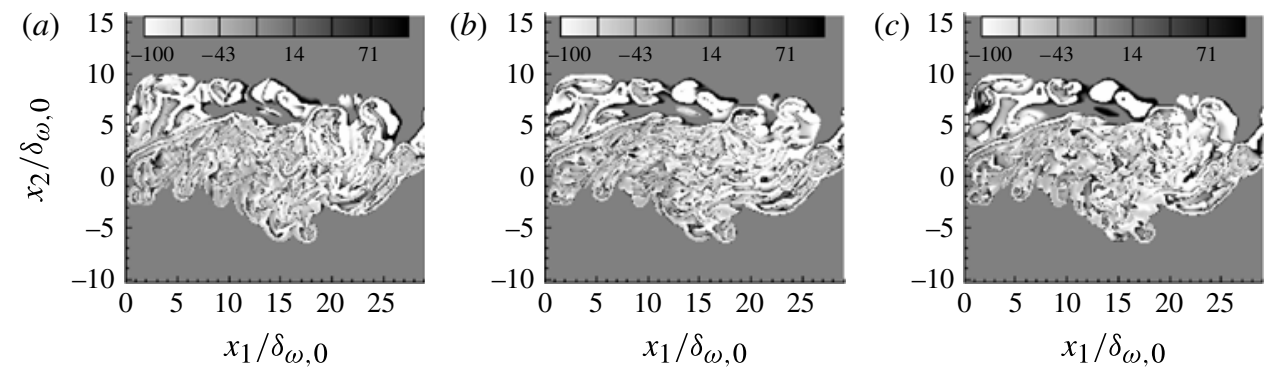

FIGURE 6. Coefficients $C_{41, x 1}(a), C_{41, x 2}(b)$ and $C_{41, x 3}(c)$ where subscripts 4 and 1 denote $\mathrm{C}_{7} \mathrm{H}_{16}$ and $\mathrm{H}_{2} \mathrm{O}$, respectively. The coefficients have been computed according to (6.22). The computation is for R1000p60 at $t_{t r}^{*}$ and in the plane $x_{3} / L_{3}=1 / 16$. The mixing layer is defined as the region where $0.01 \leqslant Y_{H e p} \leqslant 0.945$.

reaching values as large as or larger than $0.8 \times 10^{-6} \mathrm{~m}^{2} \mathrm{~s}^{-1}$ and having the largest values in the upper part of the mixing layer; intermediate values populate the entire layer while the smallest values appear at the lower boundary of the layer. Islands where $\check{D}_{H e p}$ is large at the boundary and (generally) progressively decreases towards the interior are evident in the upper stream. Here $S c_{\text {Hep,eff }}$ (figure $7 d-f$ ) follows a combined variation of $\check{D}_{H e p}, \mu$ and $\rho$; it is positive and has values as large as or larger than 1.8. Indeed, $S c_{\alpha, e f f}>1$ is expected at supercritical pressures and the $S c_{\alpha, \text { eff }}$ physically reasonable values displayed in figure 7 by the model are consistent with the excellent recovery of $J_{H e p 2}$ by the model. Clearly, not only is $S c_{\text {Hep,eff }}>1$ in most of the layer but there are $S c_{H e p, e f f} \gtrsim 1.8$ regions which mostly occur in the upper part of the layer. The fact that $S c_{\text {Hep eff }}>1$ over an overwhelming portion of the layer indicates that if the relationship between $\eta_{K}$ and $\eta_{B}$ holds according to $\eta_{B}=\eta_{K} S c_{\alpha, \text { eff }}^{-0.5}$ (the validity of this relationship has not been proved for multi-species mixing), then the good resolution of the scalar field is the criterion which must determine the grid spacing rather than the resolution of the dynamic field based on the Re value criterion (Pope 2000); whereas for atmospheric- $p$ flows it is the resolution of the dynamic field which determines the grid spacing. The realization that mixing governs the grid spacing supports the above-discussed difficulty (see §5) of finding an optimal grid for simulations in which the finest scales are due to mixing, because a quantitative criterion similar to that based on $R e$ is not available for $S c$. As $p_{0}$ increases from 60 to $80 \mathrm{~atm}, \check{D}_{\text {Hep }}$ considerably decreases whereas as $R e_{0}$ increases, $\breve{D}_{\text {Hep }}$ maintains similar values. Correspondingly, with increasing $p_{0}, S c_{\text {Hep,eff }}$ assumes values of 1.8 or larger over a larger portion of the mixing layer rather than only in isolated parts of the layer, and as $R e_{0}$ increases these $S c_{H e p, e f f} \gtrsim 1.8$ regions remain relegated mainly to the upper periphery of the layer.

A quantitative measure of the global variations of $\check{D}_{H e p}$ and $S c_{H e p, e f f}$ with $p_{0}$ and $R e_{0}$ is presented in figure 8 through the p.d.f.s computed over the entire three-dimensional mixing layer at $t_{t r}^{*}$. Figure $8(a)$ shows that $\check{D}_{\text {Hep }}$ has two peaks: the first peak is at small values which are clearly associated with regions close to the lower stream while the second peak which is at larger values of $\check{D}_{\text {Hep }}$ is more representative of the central part of the mixing layer. The largest values of $\check{D}_{\text {Hep }}$ are attributed to regions close to the upper stream. With increasing $p_{0}, \check{D}_{\text {Hep }}$ values clearly decrease and the first peak becomes now preponderant. This decrease of $\check{D}_{\text {Hep }}$ with $p_{0}$ is a new result 

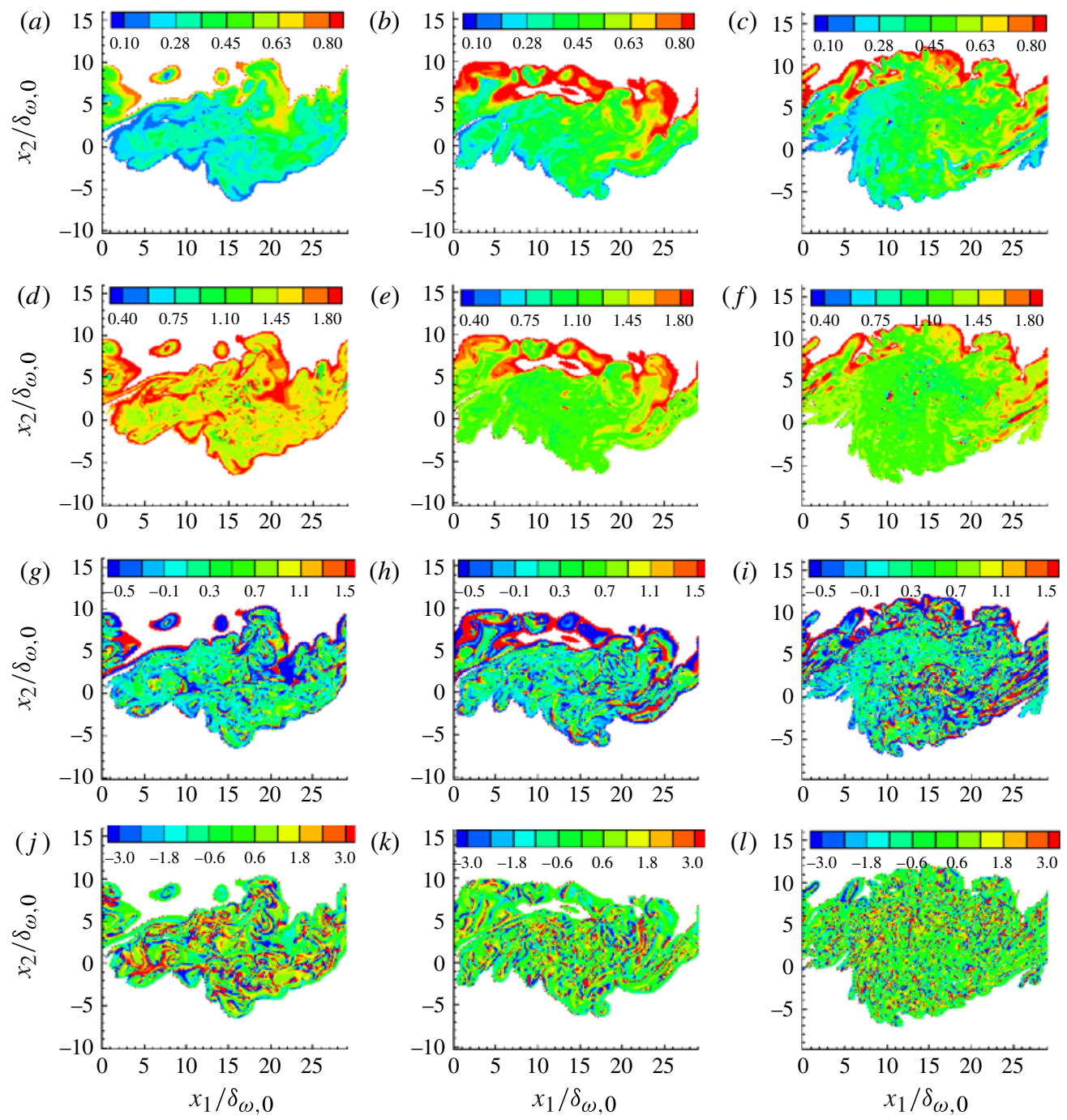

FIGURE 7. Model of $\check{D}_{\text {Hep }}(a-c), S c_{\text {Hep eff }}(d-f), \check{D}_{\text {Wat }}(g-i)$ and $S c_{\text {Wat, eff }}(j-l)$ for R1000p80 $(a, d, g, j), \mathrm{R} 1000 \mathrm{p} 60(b, e, h, k)$ and R2000p60 $(c, f, i, l)$. All at the respective $t_{t r}^{*}$ and in the plane $x_{3} / L_{3}=1 / 16$. Here $\check{D}_{\alpha}$ is not $\mathscr{F}$-scaled and units for it are $10^{-6} \mathrm{~m}^{2} \mathrm{~s}^{-1}$. The mixing layer is defined as the region where $0.01 \leqslant Y_{\text {Hep }} \leqslant 0.945$.

since we only know that $\mathscr{D}_{\alpha \gamma}$ decrease with increasing $p_{0}$ (Reid et al. 1987) but there is no theoretical development to ascertain the variation of $\mathfrak{D}_{\alpha \beta}$ with $p_{0}$, and there is no analysis to indicate the variation of coefficients such as $\check{D}_{\text {Hep }}$ with $p_{0}$. As $R e_{0}$ increases, diffusion close to the lower stream is not affected but the increased entrainment of fluid in the mixing layer slightly increases the most preponderant values there. The $S c_{H e p, \text { eff }}$ p.d.f.s displayed in figure $8(b)$ show that the distributions are also double peaked. An increase in $R e_{0}$ has almost no impact on the $S c_{\text {Hep,eff }}$ p.d.f., but an increase in $p_{0}$ shifts the p.d.f. to larger values as well as it increases the probability of a wider range of large values. The most likely values are in all three cases for $S c_{\text {Hep,eff }}>1$. For R1000p60 and R2000p60, the most likely value is $S c_{\text {Hep }, \text { eff }} \simeq 1.15$ 


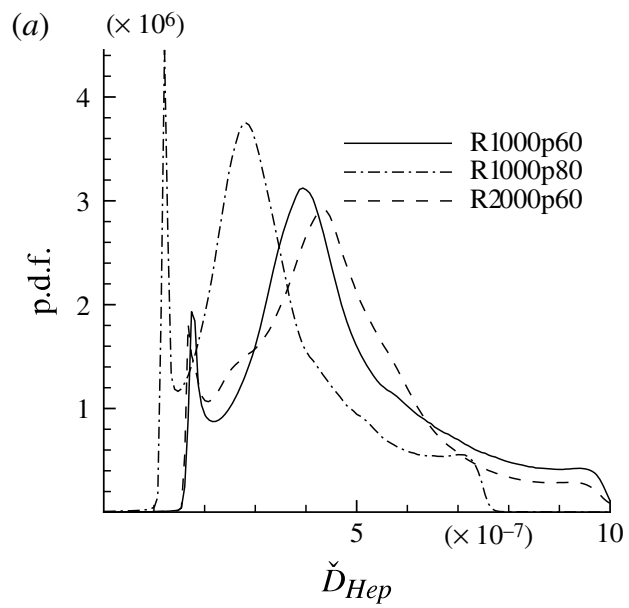

$(c) \quad\left(\times 10^{5}\right)$
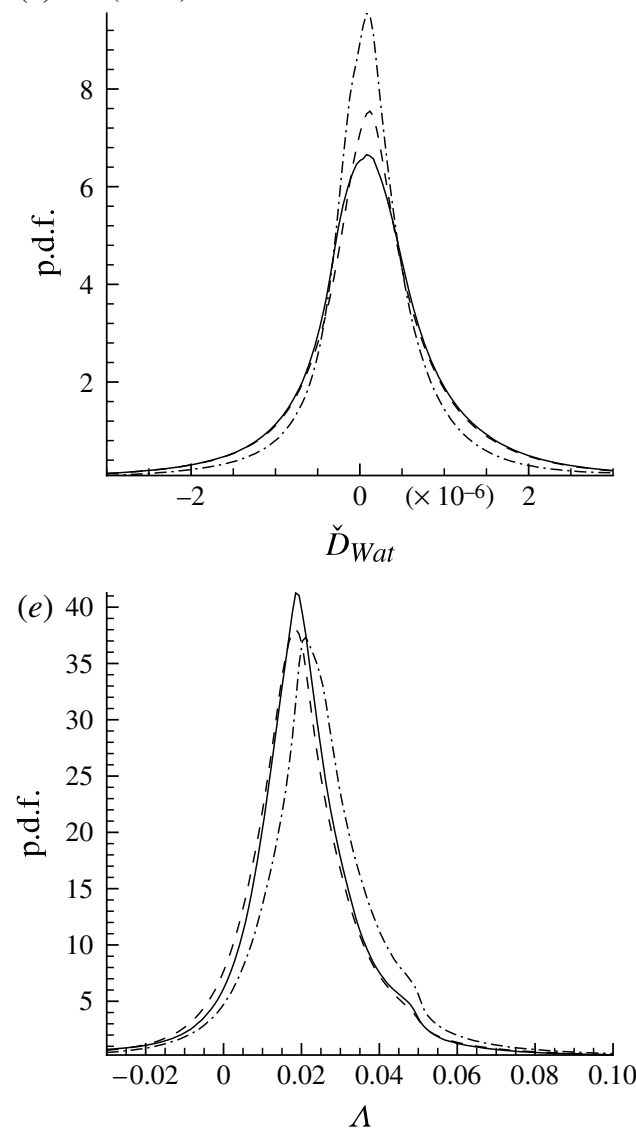

(b)

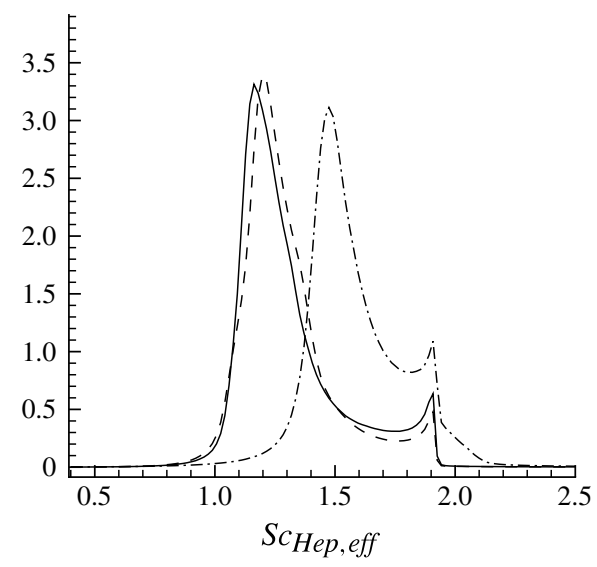

(d)

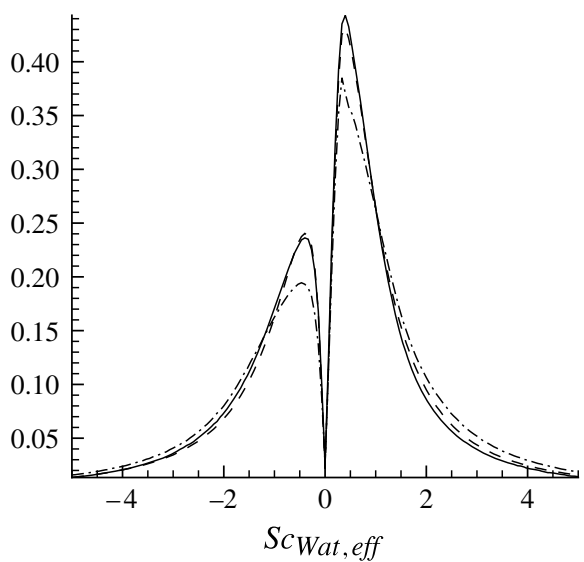

(f)

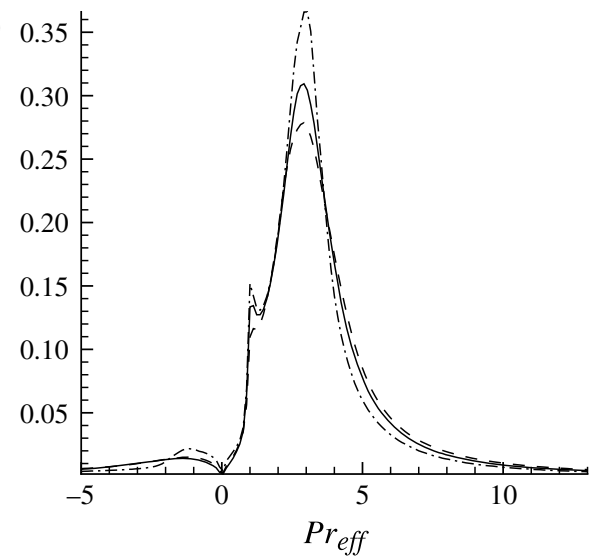

FIGURE 8. The probability density functions for R1000p60, R1000p80 and R2000p60 computed over the three-dimensional mixing layer (defined as the domain where $0.01 \leqslant$ $\left.Y_{\text {Hep }} \leqslant 0.945\right)$ at $t_{\text {tr }}^{*}$. $\check{D}_{\text {Hep }}(a), S c_{\text {Hep eff }}(b), \check{D}_{\text {Wat }}(c), S c_{\text {Wat }, \text { eff }}(d), \Lambda(e)$ and $P r_{\text {eff }}(f)$. 
whereas for $\mathrm{R} 1000 \mathrm{p} 80$ the most likely value is $S c_{\text {Hep eff }} \simeq 1.5$. For all three cases, values $S c_{\text {Hep,eff }} \leqslant 1$ have approximately the same very small probability, although the probability for R1000p80 is even smaller than for the other two cases. The likelihood of $S c_{\text {Hep ,eff }} \simeq 1.8$ doubles and the probability of $S c_{H e p, e f f} \simeq 2$ increases by more than one order of magnitude as $p_{0}$ increases from 60 to 80 atm. Values $S c_{\text {Hep ,eff }} \simeq 0.7$ are as likely as values $S c_{\text {Hep ,eff }} \simeq 2$ at $p_{0}=60 \mathrm{~atm}$ and $S c_{\text {Hep }, \text { eff }} \simeq 2.5$ at $p_{0}=80 \mathrm{~atm}$, and the probabilities are very small. The slight influence of $R e_{0}$ on these results bodes well for the applicability of these results to the range of much larger $R e$ values encountered in practical applications.

In contrast to $\breve{D}_{\text {Hep }}, \check{D}_{\text {Wat }}$ (figure $7 g-i$ ) exhibits regions of both positive and negative values; $\left|\check{D}_{\text {Wat }}\right|_{\max }>\left(\check{D}_{\text {Hep }}\right)_{\max }$ which is attributed to the $\mathrm{H}_{2} \mathrm{O}$ molecule being lighter than that of $\mathrm{C}_{7} \mathrm{H}_{16}$ (there are other considerations than molecular mass involved in determining diffusivity that are, for the sake of simplicity, not addressed here). Although not yet identified for the situation of interest in the present study, negative values of the mass-diffusion matrix elements have been measured for ternary liquid mixtures (Rehfeldt \& Stichlmair 2007, 2010). The negative values in the present situation indicate counter-gradient diffusion and are indicative of spinodal decomposition (Nauman \& He 2001; Balluffi, Allen \& Carter 2005) which is a well recognized feature of non-ideal mixtures (Nauman \& He 2001). To show that spinodal decomposition is a genuine characteristic of $\mathrm{H}_{2} \mathrm{O}$ in the system and not the result of a modelling inaccuracy as one would perhaps be tempted to conclude from figures $3(d), 4(d)$ and $5(d)$, illustrated in figure 9 are the reformulated $\check{D}_{\text {Wat }}$ computed along to the $x_{i}$ direction and the joint p.d.f.s between actual and reformulated $J_{W a t}$ for all three directions; clearly, the reformulated $\check{D}_{\text {Wat }}$ exhibits negative values in all three directions and the joint p.d.f.s show excellent agreement between the reformulated and actual values of the fluxes.

Sometimes, spinodal decomposition is also called 'phase separation' in the context of binary-species mixing, although in the present case such a terminology would be a misnomer since only $\mathrm{CO}_{2}$ and $\mathrm{H}_{2} \mathrm{O}$ display this feature; we could not find examples of multi-species mixing in the literature that would provide a guide for established terminology when such a phenomenon is observed for more than two species. To explore whether phase separation does occur for the entire mixture, a calculation of the mixture critical locus would be necessary to inquire whether at each spatial locality the mixture is in the single-phase or in the two-phase region of the thermodynamic diagram. Unfortunately, an accurate such calculation is currently unfeasible when $\mathrm{H}_{2} \mathrm{O}$ is present (T. J. Bruno, Personal communication, 4 June 2012), and thus it is postponed to a future time. Therefore, we physically interpret spinodal decomposition as a species' segregation into regions of low and high partial density (alternatively to computing the compositional field using the present governing equations, a Cahn-Hilliard-like equation (Cahn \& Hilliard 1958) could be proposed to obtain the concentration field if spinodal decomposition is suspected; but modelling diffusion and coupling to the Navier-Stokes and energy equations would still be necessary). That is, from a uniform initial composition of a species (here $\mathrm{H}_{2} \mathrm{O}$ and $\mathrm{CO}_{2}$ ), regions of larger and smaller than the initial uniform species mass fraction appear due to molecular diffusion. Because spinodal decomposition is due to molecular diffusion which occurs over the entire spatial domain (i.e. wherever there are species, temperature and/or pressure gradients), this is a process classified as a continuous transformation (Balluffi et al. 2005), unlike nucleation which is a discontinuous transformation and is due to fluctuations in thermodynamic variables 

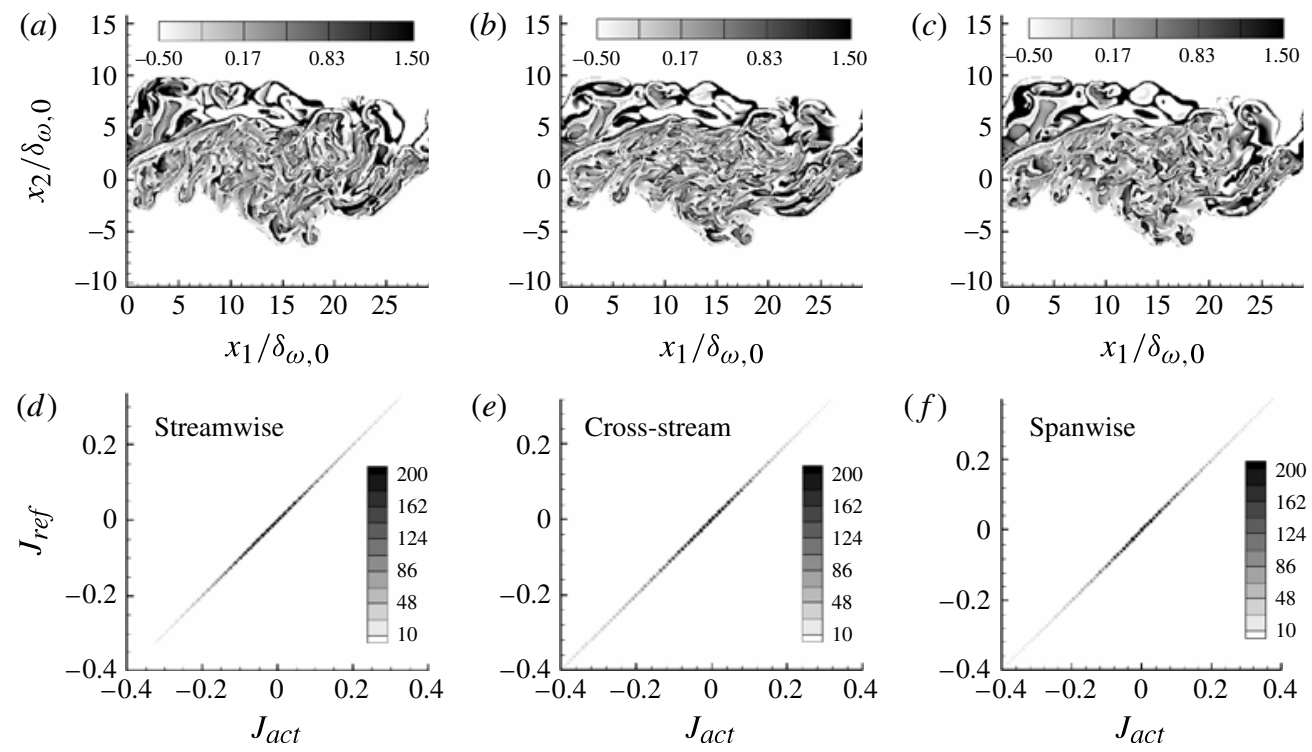

FIGURE 9. Reformulated diffusivity $\check{D}_{\text {Wat }, x i}$ computed according to (6.12), (6.19)-(6.21) and (6.22)-(6.24) (a-c); and joint p.d.f. between reformulated $J_{H e p, 2}$ using $\check{D}_{\text {Wat }, x 2}$ and actual $J_{H e p, 2}$ computed from (2.6) $(d-f)$ for R1000p60 at $t_{t r}^{*}$ and in the plane $x_{3} / L_{3}=1 / 16$. Here $\check{D}_{\text {Wat } x i}$ is not $\mathscr{F}$-scaled and units for it are $10^{-6} \mathrm{~m}^{2} \mathrm{~s}^{-1}$. The mixing layer is defined as the region where $0.01 \leqslant Y_{\text {Hep }} \leqslant 0.945$.

which occur at very localized sites. Whereas spinodal decomposition is small in degree (i.e. linear stability of the free energy) and large in spatial extent, nucleation is large in degree (i.e. nonlinear instability of the free energy) and small in spatial extent. Spinodal decomposition occurs when the composition is inside the spinodal line in the temperature-versus-composition phase diagram, whereas nucleation occurs when the composition is outside the spinodal line but still within the miscibility gap of the phase diagram. Physically, spinodal decomposition is due to the strong influence of thermodynamics on mass diffusion. Mathematically, this influence manifests through the $\alpha_{D \beta \gamma}$ matrix computed according to (2.12) and its inclusion in the computation of the mass-diffusion matrix coefficients calculated according to (2.9). The result of positive and negative values of $\breve{D}_{\text {Wat }}$ are correspondingly positive and negative values of $S c_{\text {Wat eff }}$ (figure $7 j-l$ ). For example, examining the results for R1000p60, the average value of $S c_{\text {Wat eff }}$ in the mixing layer is $\simeq 0.6$ which is smaller than the average value of $S c_{\text {Hep ,eff }} \simeq 1.2$; however the largest value of $S c_{\text {Wat eff }}$ is considerably higher than the largest local value of $S c_{H e p, e f f}$. This indicates that $\mathrm{H}_{2} \mathrm{O}$ is closer to a liquid in those regions of largest $S c_{\text {Wat eff }}$ values than is $\mathrm{C}_{7} \mathrm{H}_{16}$ in its regions of highest $S c_{\text {Hep,eff }}$ values; however, $\mathrm{H}_{2} \mathrm{O}$ is not here in liquid form since $S c$ for liquids is at least $O\left(10^{2}\right)$ for binary mixtures at standard $T$ and $p$ conditions.

The p.d.f.s of $\check{D}_{\text {Wat }}$ and $S c_{\text {Wat eff }}$ over the three-dimensional mixing layer are illustrated in figure $8(c, d)$. The Gaussian-like form of $\check{D}_{\text {Wat }}$ with the single peak located close to null values is symptomatic of the initial uniformly distributed $\mathrm{H}_{2} \mathrm{O}$ in the entire domain. Increasing $p_{0}$ from 60 to $80 \mathrm{~atm}$ results in slightly decreased $\check{D}_{\text {Wat }}$-magnitude values, whereas the doubling of the $R e_{0}$ value has practically no 

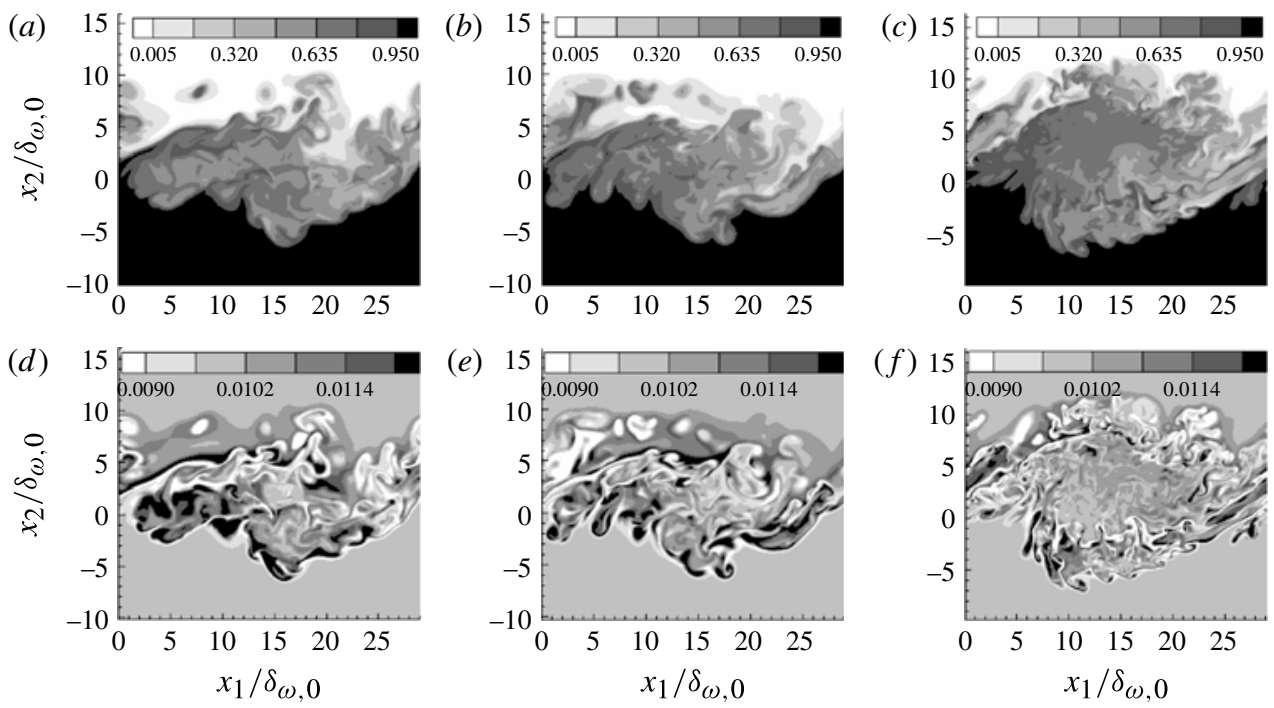

FIGURE 10. Results for $Y_{H e p}(a-c)$ and $Y_{W a t}(d-f)$ for R1000p80 $(a, d), \mathrm{R} 1000 \mathrm{p} 60(b, e)$ and $\mathrm{R} 2000 \mathrm{p} 60(c, f)$, all at the respective $t_{t r}^{*}$ and in the plane $x_{3} / L_{3}=1 / 16$.

effect on the p.d.f.. The $S c_{\text {Wat,eff }}$ p.d.f. has a wing null-centred shape. Reflecting the inverse dependency on $\check{D}_{\text {Wat }}$, null values of $S c_{\text {Wat, eff }}$ are not defined. Positive values $\simeq 0.7$ are almost twice as likely as the corresponding negative values, but at larger values the probability becomes more similar on either side of the curve. Increasing $p_{0}$ corresponds to an increase in $S c_{\text {Wat eff }}$ magnitude. Compared with the large changes for different $p_{0}$ values seen with $\check{D}_{H e p}$ and $S c_{\text {Hep } \text { eff }}$, the corresponding changes of $\check{D}_{\text {Wat }}$ and $S c_{\text {Wat eff }}$ are small. The influence of the $R e_{0}$ value on the $S c_{\text {Wat,eff }}$ p.d.f. is extremely small. Similarly to the situation for $\check{D}_{H e p}$ and $S c_{H e p \text {,eff }}$, the minor influence of $R e_{0}$ on the p.d.f.s indicates that general features of species spinodal decomposition will be encountered at the larger $R e$ values of practical interest in engines.

To show the effect of spinodal decomposition on the species composition, illustrated in figure 10 are $Y_{H e p}$ and $Y_{W a t}$ corresponding to the results shown in figure 7 . The $Y_{\text {Hep }}$ field displays a complex distribution with gradual transition between the large values in the lower portion of the layer and the small values in the upper part of the layer. In contrast, $Y_{\text {Wat }}$ displays regions of larger values than the initially uniform value of 0.01 immediately adjacent to regions of smaller values. These 'pockets' are the manifestation of species spinodal decomposition which, from the uniform initial field, produces a non-uniform field of segregated high and low concentrations. With increasing $p_{0}$, the regions of high mass fraction values appear more enlarged in area, a fact which is attributed to the more advanced species spinodal decomposition due to increasing mixture non-ideality. At larger $R e_{0}$, species spinodal decomposition occurs primarily towards the periphery of the mixing layer; the interior of the layer is conjectured to be subject to increasingly effective mixing which prevents these regions from being established (see $\S 6.2 .2 .6$ ). This finding is in concert with that of Pine et al. (1984) who experimentally found that spinodal decomposition can be suppressed in a two-species mixture by turbulent mixing. 


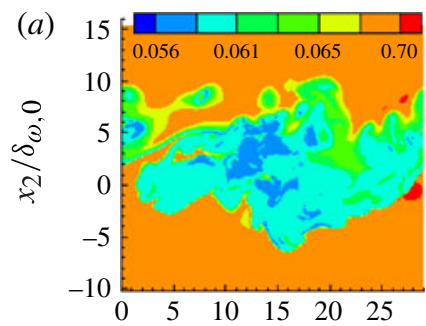

(b)
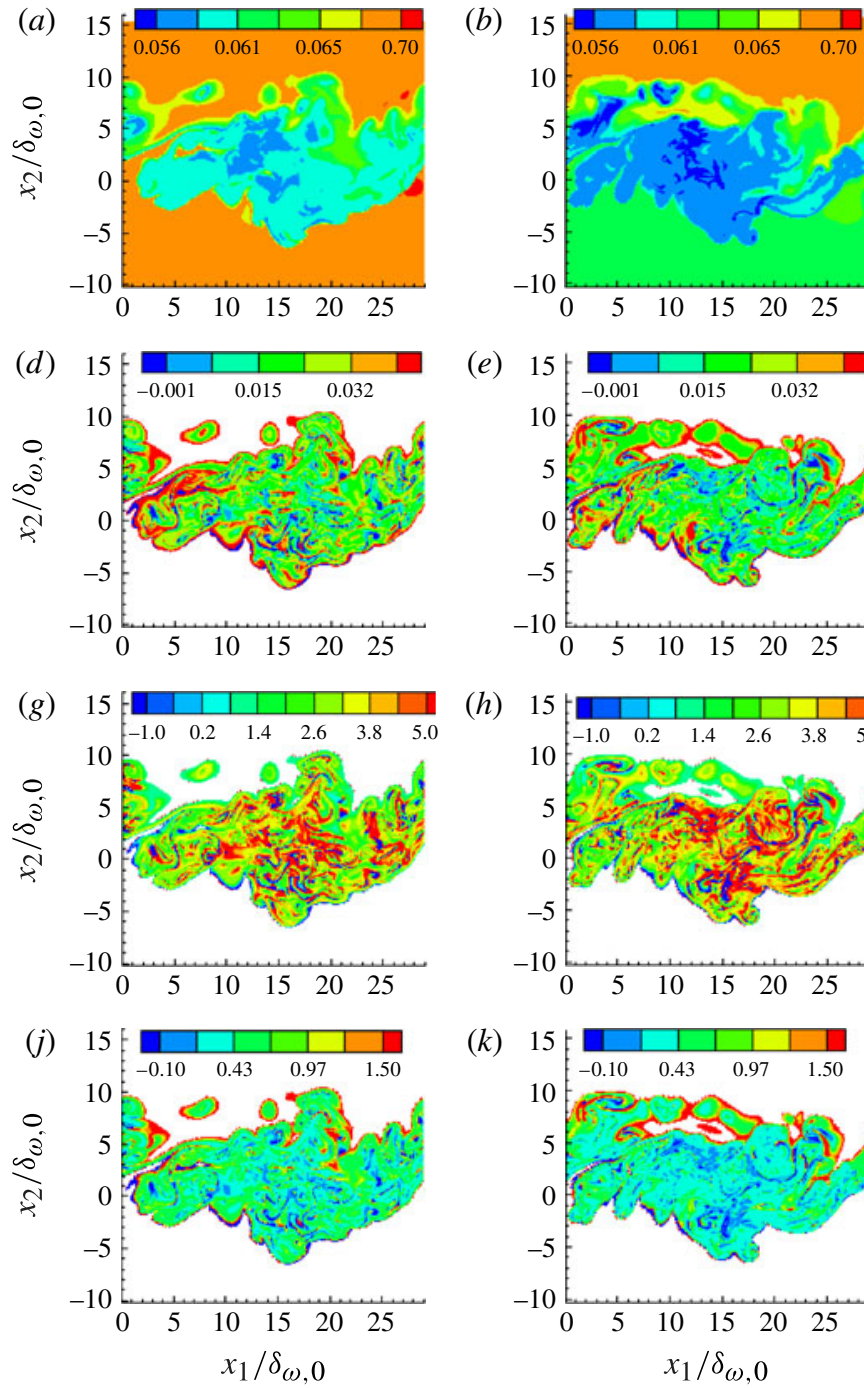

(e)

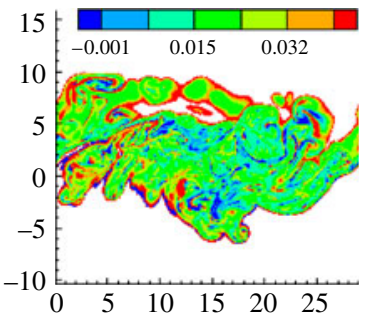

(h)

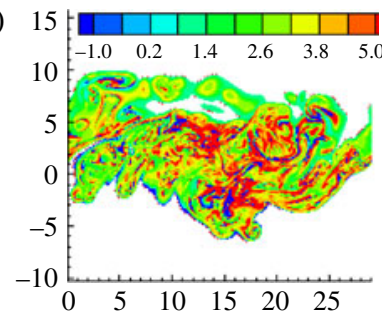

(k)

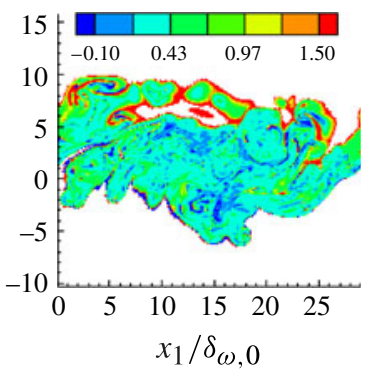

(c)

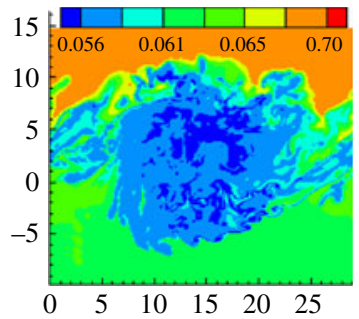

(f)

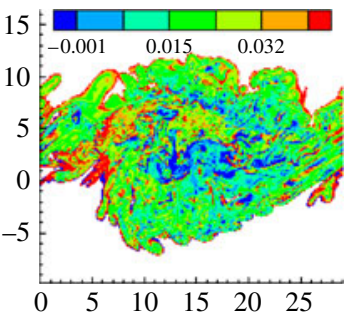

(i)

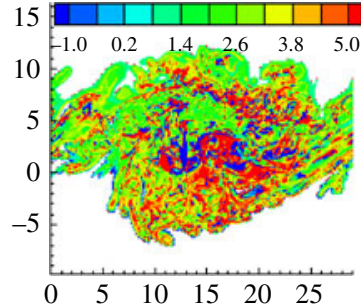

(l)

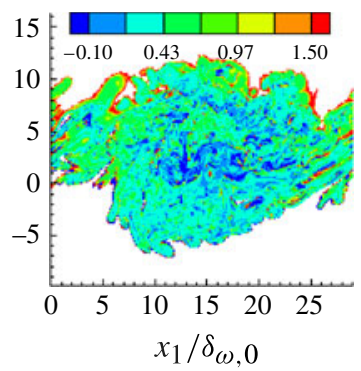

FIgURE 11. Results for $\lambda(a-c), \Lambda(d-f), \operatorname{Pr}_{\text {eff }}(g-i)$ and $L e_{\text {Hep }, \text { eff }}(j-l)$ for R1000p80 $(a, d, g, j), \mathrm{R} 1000 \mathrm{p} 60(b, e, h, k)$ and R2000p60 $(c, f, i, l)$, all at the respective $t_{t r}^{*}$ and in the plane $x_{3} / L_{3}=1 / 16$. Here $\lambda$ and $\Lambda$ are not $\mathscr{F}$-scaled and units for them are $\mathrm{W}(\mathrm{m} \mathrm{K})^{-1}$. The mixing layer is defined as the region where $0.01 \leqslant Y_{\text {Hep }} \leqslant 0.945$.

6.2.2.5. Modelling results for $\Lambda$ and $P r_{\text {eff }}$. Results obtained for $\lambda, \Lambda$ and $P r_{\text {eff }}$ are illustrated in figure 11 in the $x_{3} / L_{3}=1 / 16$ plane at $t_{t r}^{*}$. Although $\lambda$ is a positive quantity (figure $11 a-c$ ), $\Lambda$ (figure $11 d-f$ ) is mainly positive but negative values also occur in the mixing layer. The negative values are physically due to the transport of enthalpy with the species mass flux as evident in the computation of $\Lambda$ from (6.17). Physically, the effect of $\Lambda<0$ at any location is the transport of heat against the temperature gradient and thus the formation of localities of relatively low temperature within domains of high temperature or the opposite. The prevalence of the $\Lambda<0$ locations increases with increasing $\operatorname{Re}_{0}$ (compare figures $11 e$ and $11 f$ ) because of decreasing of $\lambda$ due to the mixing. Indeed, figure 11(a-c) shows that the smaller $\lambda$ 
regions are in the mixing layer. Furthermore, the larger the $R e_{0}$ value is (figures $11 b$ and 11c; figures $11 e$ and $11 f$ ), the larger is the area of the small $\lambda$ values and $\Lambda<0$ values. The prevalence of $\Lambda<0$ locations, seems, however, insensitive to increasing $p_{0}$ (cf. figure $11 e, d$ ). Since the $\lambda$ values are sensitive to $p_{0}$, the relative insensitivity of $\Lambda$ to $p_{0}$ can only be attributed to the compensating effect on $\lambda$ of the other terms in (6.17). The consequence of $\Lambda$ having both positive and negative values is that the same holds for $\operatorname{Pr}_{\text {eff }}$ (figure $11 g-i$ ) the values of which span at least $[-1,5]$ for R1000p60. These values should be compared with the well-known values of $0.7-0.8$ for air and other gases at atmospheric $p, 4-5$ for $\mathrm{R}-12$ refrigerant and approximately 7 for liquid $\mathrm{H}_{2} \mathrm{O}$. Examining figure 11 it is evident that only at sparse locations does $P r_{\text {eff }}$ have values consistent with an atmospheric- $p$ gas; in most of the domain $P r_{\text {eff }}>1$ and in a very large part of the domain $P r_{\text {eff }}$ has values similar to the high-pressure refrigerant R-12 which is a liquid. The prevalence of $P r_{\text {eff }} \approx 5$ regions appears similar or slightly reduced with increasing $p_{0}$ (compare figures $11 \mathrm{~g}$ and $11 f$ ) while they appear similar or slightly increased with increasing $\operatorname{Re}_{0}$ (compare figures $11 \mathrm{~h}$ and $11 \mathrm{i}$ ) meaning that such $\operatorname{Pr}_{\text {eff }}$ values are almost certainly encountered under realistic engine conditions. The fact that $P r_{\text {eff }}>1$ over a large portion of the mixing layer supports our display of $E(T)$ in figure 2 since it is clear that in these simulations the resolution is dictated by the mixing and thermal scales.

Finally, of interest is the effective Lewis number, $L e_{\text {eff }}$; since $\check{D}_{\alpha}$ varies according to the species, $L e_{\alpha, e f f} \equiv S c_{\alpha, e f f} / P r_{\text {eff }}$. Depicted in figure $11(j-l)$ is $L e_{H e p, e f f}$. In contrast to $L e_{e f f}$ computed for supercritical binary-species mixing by Harstad \& Bellan (1999) in the context of conservation equations satisfied by defined dependent variables which were combinations of $Y_{\alpha}$ and $T$ that had the property of diagonalizing the set of equations, the present $L e_{\alpha, e f f}$ is for the same set of variables as the original system of equations, and is thus more relevant. Values of $L e_{H e p, e f f}$ range from a minimum of at least -0.1 to an excess of 1.5 ; negative values are more prominent for the larger $R e_{0}$. As $p_{0}$ increases, so does the average value of $L e_{H e p, e f f}$ computed over the three-dimensional mixing layer (not shown).

An enhanced understanding of the $\Lambda$ and $P r_{\text {eff }}$ range of values can be obtained by examining figure $8(e, f)$ showing the p.d.f.s of these quantities over the threedimensional mixing layer at $t_{t r}^{*}$. The p.d.f. of $\Lambda$ is single peaked with the peak centred approximately at 0.02 . Positive values are most likely, but negative values (e.g. -0.02) are as possible as larger than 0.02 positive values (i.e. 0.06 ). The doubling of $R e_{0}$ has almost no impact on the $\Lambda$ p.d.f., but an increase of $33 \%$ in $p_{0}$ shifts the p.d.f. to slightly larger values. The p.d.f. of $P r_{\text {eff }}$ has a complex shape but in all three cases the most likely value is $\simeq 3$. A gas-like value of 0.7 is approximately as likely as the value 7 and the value of 1 is almost as likely as a value of 5, showing that the mixture has characteristics far from the well-studied atmospheric flows. Increasing $R e_{0}$ increases imperceptibly the probability of finding $\operatorname{Pr}_{\text {eff }}$ larger positive values while increasing $p_{0}$ has the opposite effect. The p.d.f. of $L e_{\text {Hep,eff }}$ (not shown, for brevity) is centred at approximately 0.3 for R1000p60 and R2000p60, and at 0.5 for R1000p 80 . The value of 1 is approximately as likely as that of -0.2 for R1000p60 and R2000p60 and as likely as 0 for R1000p80, indicating the unconventional nature of the situation with respect to atmospheric flows. Given the very low sensitivity of these results to $R e_{0}$, this unconventional nature is conjectured to persist at much larger $R e$ values unattainable in DNS.

The corresponding $T$ distribution in the $x_{3} / L_{3}=1 / 16$ plane and the temperature fluctuations $\left(T^{\prime}=T-\langle T\rangle\right)$ in the mixing layer of that plane (not meaningful elsewhere 

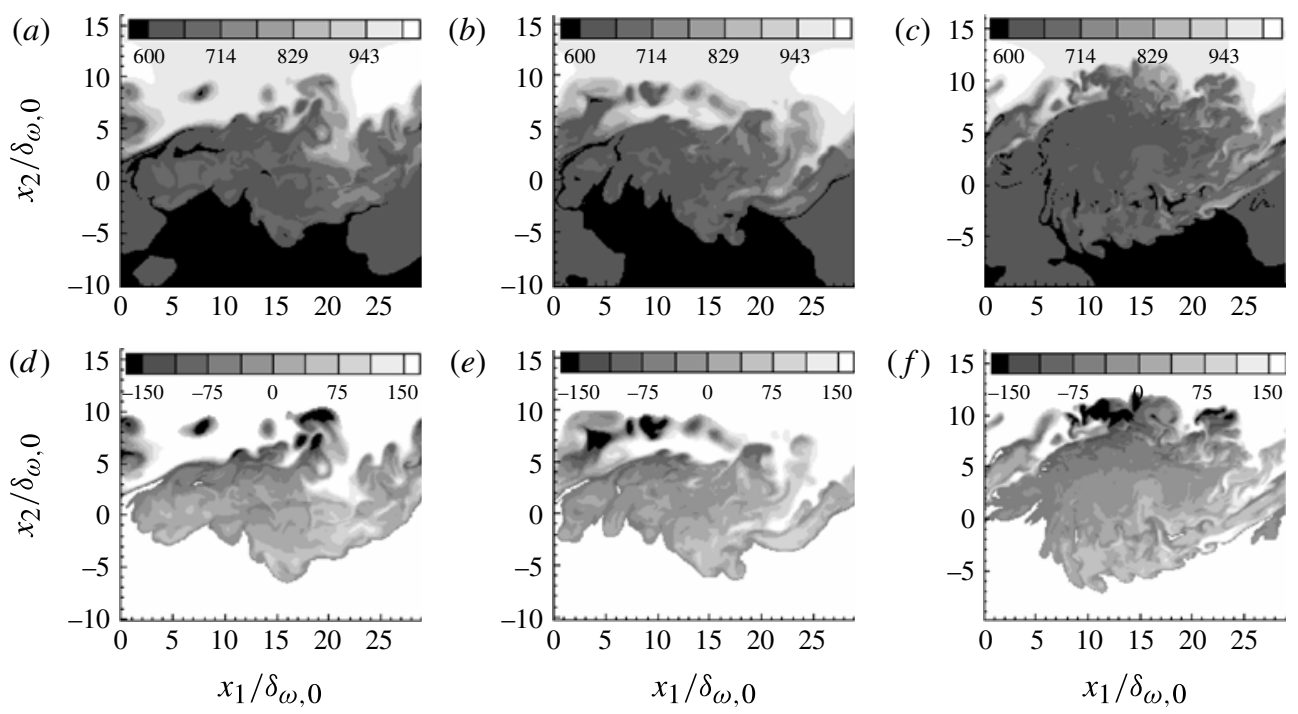

FIGURE 12. Temperature $T$ in the entire plane $(a-c)$ and $T^{\prime}=T-\langle T\rangle$ in the mixing layer $(d-f)$ for R1000p80 $(a, d), \mathrm{R} 1000 \mathrm{p} 60(b, e)$ and R2000p60 $(c, f)$, all at the respective $t_{t r}^{*}$ and in the plane $x_{3} / L_{3}=1 / 16$. Units are in Kelvin. For $(d-f)$, the mixing layer is defined as the region where $0.01 \leqslant Y_{\text {Hep }} \leqslant 0.945$.

in the domain) are portrayed in figure 12. Clearly, in all of the illustrated flows there are mixing layer regions having pockets of small temperature, some where $T<600 \mathrm{~K}$, interspersed in a field where $T$ is much larger. The formation of pockets of large and small $T$ would clearly be undesirable in the cold-ignition regime which motivated this study because the intent is to facilitate heat transfer from hot air to the injected cold fuel so as to help ignite the mixture.

At this junction, two remarks are in order for $\S 6.2 .2 .4$ and $\S 6.2 .2 .5$. First, although the presented results were at the respective $t_{t r}^{*}$ of the simulations, examination of the states produced by the layers past the first pairing (not shown) exhibit the same features as the transitional states in terms of spinodal decomposition and its consequences. Second, it is clear that the $\check{D}_{\alpha}$ quantitative features are primarily dependent on the value of $p_{0}$ and are almost independent of the $R e_{0}$ value, a fact which bodes well for the relevance of future studies using the DNS database for LES modelling. Since the model for $\Lambda$ is based on that for $\check{D}_{\alpha}$, that is, on small-scale gradient processes, the quantitative change of $\Lambda$ must necessarily be included in LES using subgrid-scale modelling as otherwise they would be absent at the LES grid-spacing resolution. Such subgrid-scale models do not currently exist and must be the subject of future studies.

6.2.2.6. Necessary and sufficient condition for obtaining species spinodal decomposition. The above analysis shows that spinodal decomposition occurs for $\mathrm{H}_{2} \mathrm{O}$ (and $\mathrm{CO}_{2}$; not shown) but not for $\mathrm{C}_{7} \mathrm{H}_{16}$ (and $\mathrm{N}_{2}$ or $\mathrm{O}_{2}$; not shown). To understand the conditions which promote spinodal decomposition and those which prevent it, two additional simulations were conducted such that in one of them (R600p60*) $\mathrm{H}_{2} \mathrm{O}$ and $\mathrm{CO}_{2}$ were initially present only in the upper stream, while in the other simulation (R600p60**) $\mathrm{H}_{2} \mathrm{O}$ and $\mathrm{CO}_{2}$ were present initially in both streams, but, in contrast 

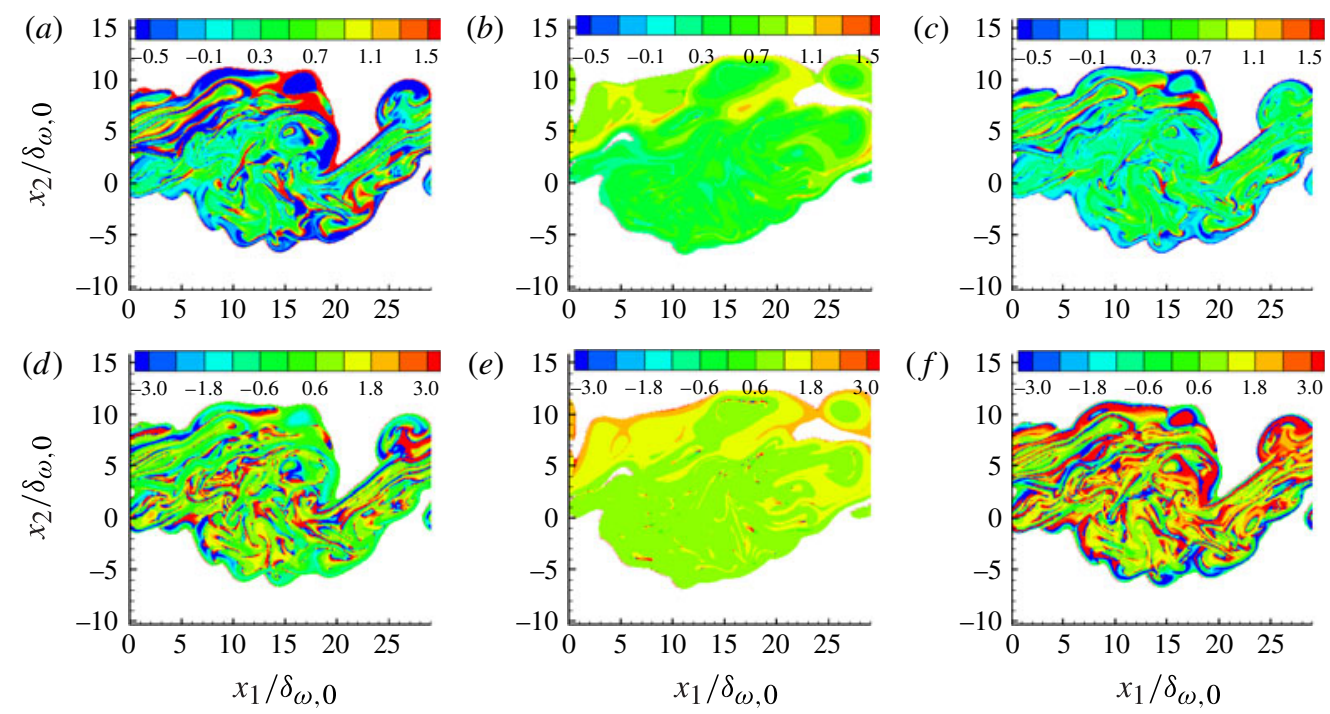

FIGURE 13. Model of $\check{D}_{W a t}(a-c)$ and $S c_{W a t, e f f}(d-f)$ for R600p60 $(a, d)$, R600p60* $(b, e)$ and R600p60** $(c, f)$, all at the respective $t_{t r}^{*}$ and in the plane $x_{3} / L_{3}=1 / 16$. Here $\check{D}_{\text {Wat }}$ is not $\mathscr{F}$-scaled and units for it are $10^{-6} \mathrm{~m}^{2} \mathrm{~s}^{-1}$. The mixing layer is defined as the region where $0.01 \leqslant Y_{\text {Hep }} \leqslant 0.945$.

to R600p60, each species mass fraction had a different value in the lower stream and in the upper stream (see table 2). Simulation R600p60* is very similar in character (values of $\left(\rho_{L} / \rho_{U}\right)_{0}$, $t_{t r}^{*}$, etc.) to the binary species $\mathrm{C}_{7} \mathrm{H}_{16}-\mathrm{N}_{2}$ DNS conducted by Okong'o \& Bellan (2002a) since $\mathrm{H}_{2} \mathrm{O}$ and $\mathrm{CO}_{2}$ are only present mixed with air in the upper stream and have very small mass fractions, while only pure fuel is present in the lower stream. Comparisons of the results for $\check{D}_{\text {Wat }}$ and $S c_{\text {Wat,eff }}$ appear in figure 13 at the respective $t_{t r}^{*}$. The evidence is that, as indicated by the sign of $\check{D}_{\text {Wat }}$, spinodal decomposition of $\mathrm{H}_{2} \mathrm{O}$, and generally of a species, only occurs if that species is not initially segregated into one stream, independent of whether the distribution of that species is initially uniform over the entire domain. When $\mathrm{H}_{2} \mathrm{O}$ does not experience spinodal decomposition, as is the case for R600p60*, $S c_{\text {Wat,eff }}$ generally varies at least in the range $[0.5,1.5]$ confirming the fitting for $S c$ as a function of $Y_{H e p}$ (i.e. $S c=1.5-Y_{H e p}^{2}$ ) in the binary species $\mathrm{C}_{7} \mathrm{H}_{16}-\mathrm{N}_{2}$ DNS performed by Okong'o \& Bellan (2002a). When spinodal decomposition is obtained, $\check{D}_{W a t}$ is considerably more non-uniform and assumes more extreme values when the initial composition of $\mathrm{H}_{2} \mathrm{O}$ is initially uniform than when it is not, whereas the opposite occurs for $S c_{\text {Wat, eff }}$. In both R600p60 and R600p60** cases, values of $S c_{\text {Wat,eff }}$ at least as small as -3 and at least as large as 3 (figure $13 d, f$ ) occur throughout the flow in contrast to the much reduced range of values, from 0.5 to 1.5 , for R600p60* (figure 13e).

Since R600p60* reached transition considerably later than R600p60 and R600p60**, it is clear that not only are the effective transport properties affected by spinodal decomposition but that the vortical aspects of the flow are also modified by its occurrence. Measures of small-scale creation and flow topological complexity are $\left\langle\left\langle\omega_{3}^{+}\right\rangle\right\rangle \delta_{\omega, 0} / \Delta U_{0}$ and $\left\langle\left\langle\omega_{i} \omega_{i}\right\rangle\right\rangle\left(\delta_{\omega, 0} / \Delta U_{0}\right)^{2}$, respectively, both of which are illustrated versus $t^{*}$ in figure 14 . The indication is that R600p60* has reduced small-scale and 

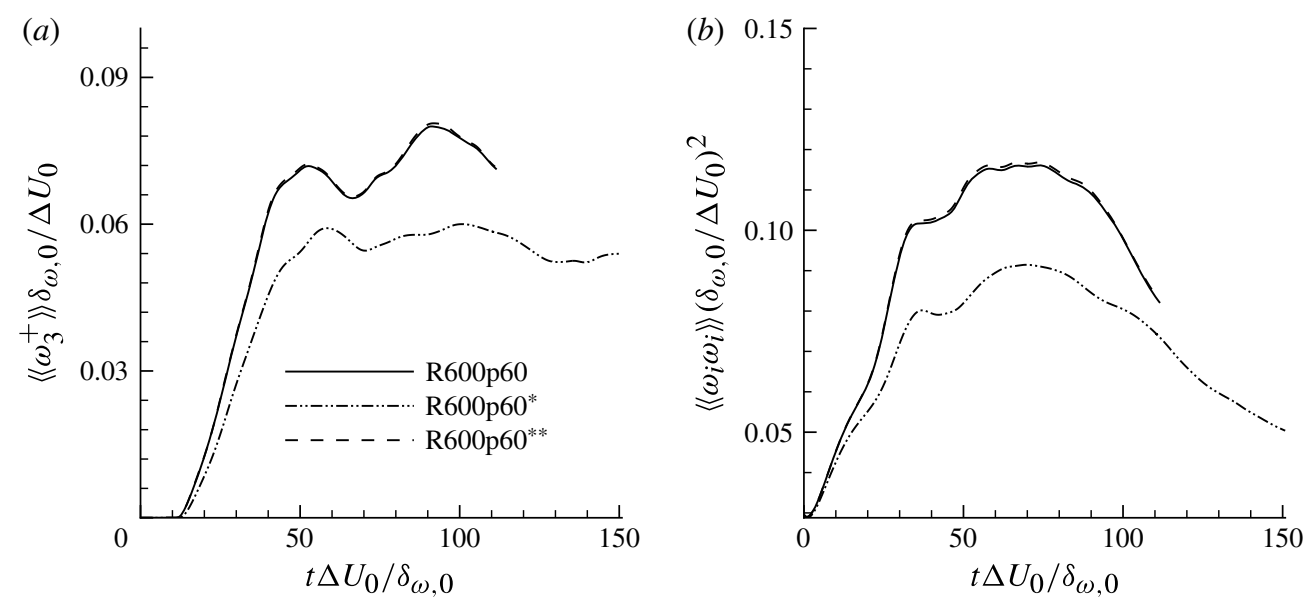

FIGURE 14. Measures of small-scale creation and flow topological complexity $\left\langle\left\langle\omega_{3}^{+}\right\rangle\right\rangle \delta_{\omega, 0} / \Delta U_{0}(a)$ and $\left\langle\left\langle\omega_{i} \omega_{i}\right\rangle\right\rangle\left(\delta_{\omega, 0} / \Delta U_{0}\right)^{2}(b)$, respectively, for R600p60, R600p60* and R600p60** listed in table 1.

topological complexity compared with R600p60 and R600p60**. We attribute the more turbulent aspects of the spinodal-decomposition-affected layers to the increased compositional, and thus of density, non-uniformities (not shown); indeed, experimental evidence from Hannoun, Fernando \& List (1988) shows that the large-magnitude $|\nabla \rho|$ regions act similarly to hard boundaries and modify the turbulent field by redistributing turbulence and increasing the turbulence anisotropy of the large scales. Basically, the large-magnitude $|\nabla \rho|$ regions (see $\S 6.2 .3$ for discussions on $|\nabla \rho|$ ) act in the flow similarly to a solid mesh which increases turbulence. Therefore, while spinodal decomposition inherently hinders mixing it also promotes turbulence which increases mixing. The result of these coupled counteracting processes is complex and not predictable without simulations.

6.2.2.7. Extended significance of the reformulated equations and of the $S c_{\alpha, e f f}$ and $P r_{\text {eff }}$ models. The above derivation and the successful models for $S c_{\alpha, e f f}$ and $P r_{\text {eff }}$ show that even for the complex form of the species and heat fluxes under consideration (equations (2.6) and (2.7)) which couple all thermodynamic variables, it is possible to effectively diagonalize the system of differential equations for these variables. This diagonalization does not stem from uncoupling the system of equations; that is, this is not a diagonalization of the complete flux matrix, a procedure which in the best of cases would only be possible at each location and thus eigenvectors would be location dependent, an attribute that would not be very useful. The uncoupling of the flux matrix through diagonalization would have entailed a change of dependent variables, whereas the present procedure maintains the original set of dependent variables. In contrast to what might have been expected from the instinctive idea that the set of equations can only be diagonalized by uncoupling them, the present diagonalization takes advantage of the natural coupling among all variables and therefore obeys physics in an innate way. Also, the type of diagonalization proposed here is always feasible in regions of non-null gradients, whereas the uncoupling-type diagonalization may not always be possible. The present formalism for diagonalization is exact, but a model is required to actually obtain values of the quantities resulting 
from the diagonalization (a model would not have been necessary for an uncoupling through change-of-dependent-variables diagonalization); the model developed above was shown to be very accurate. This strategy for diagonalizing the system of equations may be very helpful when attempting to use the flamelet model of Peters (2000) in multi-species combustion simulations if conserved scalars can be defined through combinations of the species mass fractions that allow the elimination of reaction terms and if $L e_{\alpha, e f f} \approx 1$.

Not only is the developed model of $\check{D}_{\alpha}$ permitting insights into the transport processes occurring in non-ideal mixtures, but it also allows evaluation of some assumptions that are of interest for combustion simulations. Such an evaluation is presented in appendix $\mathrm{C}$ and shows that although the diffusion matrix $\mathfrak{D}_{\alpha \beta}$ is diagonally dominated, neglect of the off-diagonal terms results in missing the physics of species spinodal decomposition as well as using diffusion coefficients of qualitatively incorrect spatial distribution and of quantitatively erroneous range of values.

\subsubsection{Thermodynamic characteristics of the mixture}

The above analysis showed the strong influence of the thermodynamics on the compositional, thermal and dynamic features of the layer and thus motivated farther examination of these aspects.

Okong'o \& Bellan (2002a) have shown that for binary species $\mathrm{C}_{7} \mathrm{H}_{16}-\mathrm{N}_{2}$ mixing, regions of large $|\nabla \rho|$ were formed and that for $R e_{0}=600$ the typical magnitude of $|\nabla \rho|$ was of the order of $10^{4} \mathrm{~kg} \mathrm{~m}^{-4}$. For the same $R e_{0}=600$, when $\mathrm{H}_{2} \mathrm{O}$ and $\mathrm{CO}_{2}$ are present in trace amounts with compositions initially uniform, leading to their spinodal decomposition, similar values to those of Okong'o \& Bellan (2002a) are obtained according to the illustration of figure 15(a). With increasing $R e_{0}$ values (compare figure $15(a-c, e)$ the regions of higher $|\nabla \rho|$ values become more prevalent as well as more convoluted and penetrate further within the layer. When $p_{0}$ increases (compare figure $15 b, d, f$ ), the sharpness of the $|\nabla \rho|$ regions increases.

In figure 15 , the thick black line denotes regions where $0.99 \leqslant E R \leqslant 1.01$, corresponding to a thin equivalence-ratio band centred at the stoichiometric value. In all cases, the stoichiometric region is thin compared with other features of the flow indicating that if this aspect prevails for reactive flows, the flow will be in the flamelet regime (Williams 1975) and the flamelet model (Peters 2000) could perhaps be used in the future. The stoichiometric region is at the upper-stream limit of the mixing layer, at the boundary where $\mathrm{C}_{7} \mathrm{H}_{16}$ meets $\mathrm{O}_{2}$, but isolated pockets of stoichiometric mixture also penetrate the interior of the layer. This conceptual picture is independent of the $R e_{0}$ and $p_{0}$ values. Some islands of stoichiometric mixing enclose regions of very intense mixing (see figure $15 d, f$ ). Figure $16(a-c)$ show that these stoichiometric regions fall thermodynamically in regions where the compression factor, $Z=p /\left(\rho T R_{u} / m\right) \simeq 1$, with $Z$ being one measure of the departure from perfect gas for which $Z=1$. However, the mixture is not a perfect gas there because in addition to $Z=1$, the partial pressure of species $\alpha, p_{\alpha}$, must satisfy $p_{\alpha} / p=X_{\alpha}$. According to the findings of $\S 6.2 .2$, the mixture is thus non-ideal in the stoichiometric region. Independent of $R e_{0}$ and $p_{0}$ values, in most of the layer $Z$ ranges between 0.9 and 1, although locations with $Z$ as small as 0.6 can also be seen within the layer. For example, for all three simulations illustrated in figure 16, the regions of $Z \simeq 0.6$ directly and predominantly correspond to those where $T \lesssim 600 \mathrm{~K}$ within the layer (compare figure $16 a-c$ with figure $12 a-c$ ), highlighting the importance of departures from perfect gas behaviour during supercritical- $p$ species mixing. In the 

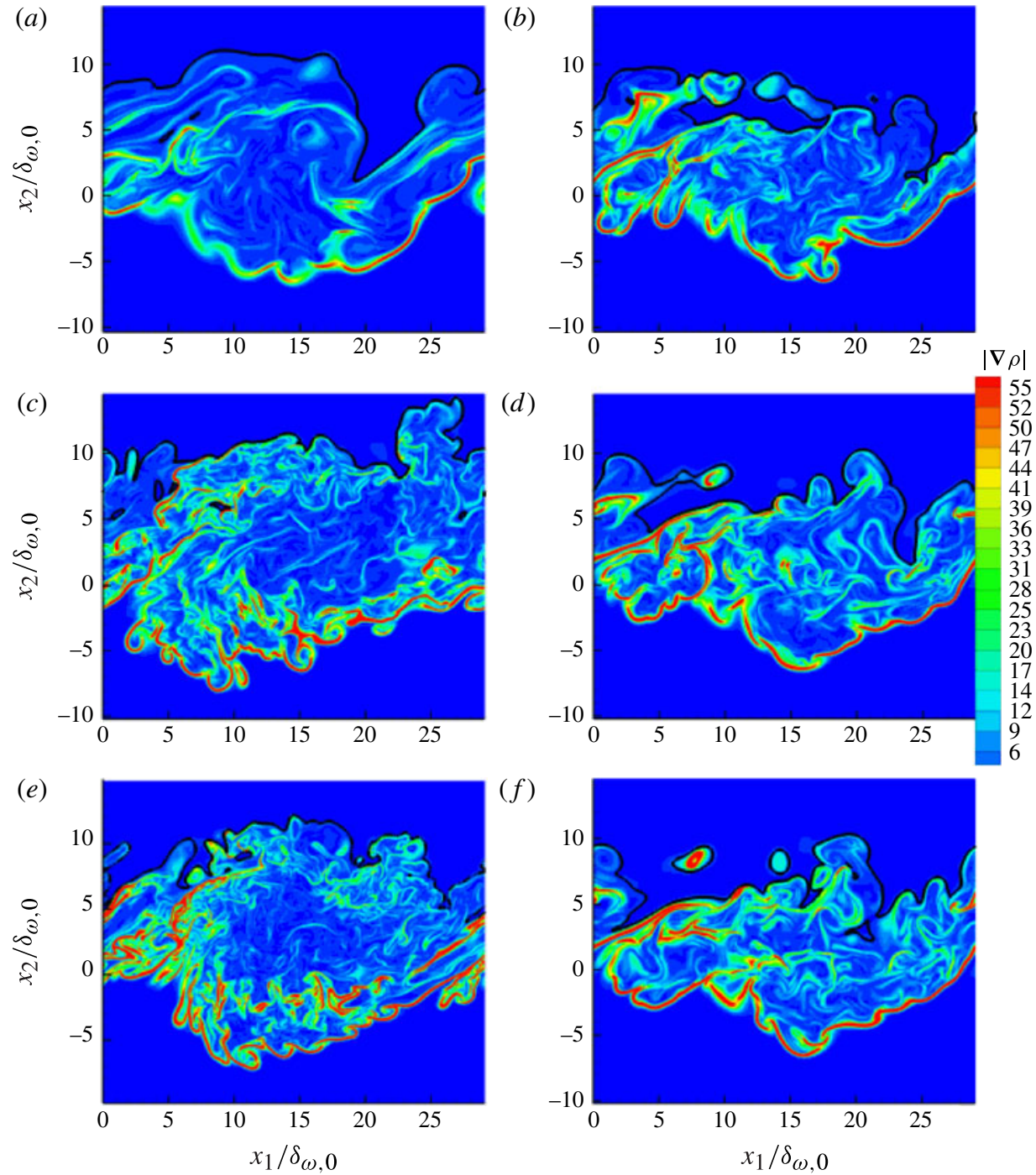

FIGURE 15. Results for $|\nabla \rho|$ in $10^{3} \mathrm{~kg} \mathrm{~m}^{-4}$ for R600p60 (a), R1000p60 (b), R1600p60 $(c), \mathrm{R} 1000 \mathrm{p} 70(d), \mathrm{R} 2000 \mathrm{p} 60(e)$ and R1000p80 $(f)$, all at the respective $t_{t r}^{*}$ and in the plane $x_{3} / L_{3}=1 / 16$. The black line in each figure represents the equivalence ratio region $0.99 \leqslant E R \leqslant 1.01$.

lower stream, $Z \simeq 0.56$ for $p_{0}=60$ atm and $Z \lesssim 0.53$ for $p_{0}=80$ atm. Moreover, examination of figure 15 in conjunction with figure 11 shows that in the stoichiometric region $L e_{\text {Hept } \text { eff }} \gtrsim 1.5$ (1.5 is the upper value of the plotted range), indicating that if this aspect persists for reactive flows, a conventional flamelet model (Peters 2000) may not be applicable.

According to figure $16(d-f)$, regions of $E R$ as large or exceeding 8.0 and as small or smaller than 0.01 are found in close proximity to the stoichiometric region; the plots were made by eliminating regions of $E R<0.01$ which dominate the upper stream. Independent of the $R e_{0}$ and $p_{0}$ values, regions of very small $E R$ coexist with regions of very large $E R$ illustrating the complexity of the composition. As $R e_{0}$ increases, the spatial extent of the region included in the range $[0.01-4]$ decreases. 

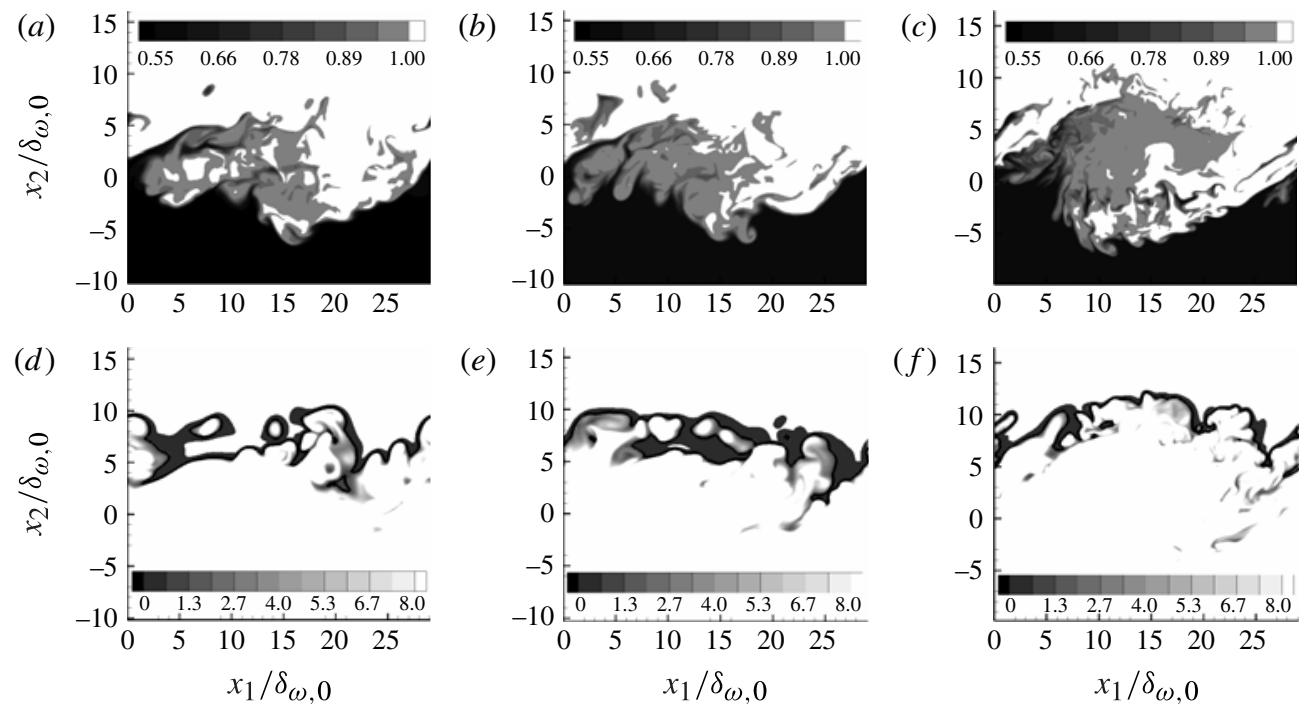

FIGURE 16. Results for $Z(a-c)$ and $E R(d-f)$ for R1000p80 $(a, d)$, R1000p60 $(b, e)$ and R2000p60 $(c, f)$, all at the respective $t_{t r}^{*}$ and in the plane $x_{3} / L_{3}=1 / 16$. The black line represents the equivalence ratio region $0.95 \leqslant E R \leqslant 1.05$.

\subsection{Irreversible entropy production}

Since the quantity that includes all transport effects is the dissipation, this quantity is here examined to further elucidate the impact of the intertwined influence of thermodynamics and transport properties on the flow. The dissipation is the irreversible entropy production which is the source term in the entropy equation.

The entropy conservation equation has been derived in the past (e.g. de Groot \& Mazur 1984) but that formulation does not account for the transport coefficient calculation through the mixing rules. In the present context, if $S$ is the entropy per unit mass,

$$
\frac{\partial(\rho S)}{\partial t}+\frac{\partial\left(\rho S u_{j}\right)}{\partial x_{j}}=-\frac{\partial \Sigma_{j}}{\partial x_{j}}+g
$$

where $\Sigma_{j}$ represents the flux of entropy and $g$ is the rate of irreversible entropy production which is the focus of our interest. From thermodynamics,

$$
T \frac{\mathrm{D} S}{\mathrm{D} t}=\frac{\mathrm{D} e}{\mathrm{D} t}+p \frac{\mathrm{D}(1 / \rho)}{\mathrm{D} t}-\sum_{\alpha=1}^{N} \frac{\mu_{\alpha}}{m_{\alpha}} \frac{\mathrm{D} Y_{\alpha}}{\mathrm{D} t}
$$

so that

$$
\rho \frac{\mathrm{D} S}{\mathrm{D} t}=\frac{\rho}{T} \frac{\mathrm{D} e}{\mathrm{D} t}+\left(-p+\sum_{\alpha=1}^{N} \frac{\mu_{\alpha} \rho Y_{\alpha}}{m_{\alpha}}\right) \frac{1}{\rho T} \frac{\mathrm{D} \rho}{\mathrm{D} t}-\sum_{\alpha=1}^{N} \frac{\mu_{\alpha}}{T m_{\alpha}} \frac{\mathrm{D} \rho Y_{\alpha}}{\mathrm{D} t},
$$

which leads to

$$
\Sigma_{j}=\frac{1}{T}\left(-q_{j}+\sum_{\alpha=1}^{N} \mu_{\alpha} \frac{J_{\alpha j}}{m_{\alpha}}\right)
$$


and

$$
g=\underbrace{\frac{\mu}{T}\left(2 S_{i j} S_{i j}-\frac{2}{3} S_{k k} S_{l l}\right)}_{g_{\text {visc }}}-\underbrace{\sum_{\text {gmass ffux }^{N}}^{\sum_{\alpha=1}^{N} \frac{J_{\alpha j}}{m_{\alpha}} \frac{\partial}{\partial x_{j}}\left(\frac{\mu_{\alpha}}{T}\right)} .}_{\text {gheat }^{\frac{q_{j}}{T^{2}} \frac{\partial T}{\partial x_{j}}}}
$$

Part of the third term in (6.32) can be recast using

$$
\frac{\partial}{\partial x_{j}}\left(\frac{\mu_{\alpha}}{T}\right)=\frac{1}{T}\left(-s_{\alpha} \frac{\partial T}{\partial x_{j}}+v_{\alpha} \frac{\partial p}{\partial x_{j}}+\sum_{\beta=1}^{N} \frac{\partial \mu_{\alpha}}{\partial X_{\beta}} \frac{\partial X_{\beta}}{\partial x_{j}}\right)-\frac{\mu_{\alpha}}{T^{2}} \frac{\partial T}{\partial x_{j}}
$$

where $s_{\alpha}$ is the partial molar entropy. Further manipulation of (6.33) taking into account that the chemical potentials are the partial molar Gibbs functions, $\mu_{\alpha}=h_{\alpha}-T s_{\alpha}$, yields

$$
\frac{\partial}{\partial x_{j}}\left(\frac{\mu_{\alpha}}{T}\right)=-h_{\alpha} \frac{1}{T^{2}} \frac{\partial T}{\partial x_{j}}+\frac{1}{T} v_{\alpha} \frac{\partial p}{\partial x_{j}}+\sum_{\beta=1}^{N}\left(\delta_{\alpha \beta}+X_{\alpha} R_{\alpha \beta}\right) \frac{R_{u}}{X_{\alpha}} \frac{\partial X_{\beta}}{\partial x_{j}}
$$

where $R_{\alpha \beta}$ is defined by (2.13). Additional mathematical manipulations lead to the quadratic form of $g$ written as the addition of three terms accounting for contributions from viscous, thermal and species transport, $g_{\text {visc }}, g_{\text {temp }}$ and $g_{\text {mass }}$

$$
g=\underbrace{\frac{\mu}{T}\left(2 S_{i j} S_{i j}-\frac{2}{3} S_{k k} S_{l l}\right)}_{\text {g visc }}+\underbrace{\lambda \frac{1}{T^{2}} \frac{\partial T}{\partial x_{j}} \frac{\partial T}{\partial x_{j}}}_{\text {gtemp }}+\underbrace{\frac{1}{2} \sum_{\beta=1}^{N} \sum_{\alpha=1}^{N} \frac{R_{u}}{\left(-\mathbb{D}_{\alpha \beta}\right)} \frac{\rho}{m_{\alpha} Y_{\beta}} \Pi_{\alpha \beta j} \Pi_{\alpha \beta j}}_{\text {gmass }}
$$

where $\left(\left(-\mathbb{D}_{\alpha \beta} m\right) /\left(Y_{\alpha} m_{\beta}\right)\right)$ is a symmetric positive semi-definite matrix (Keizer 1987; Giovangigli et al. 2011) and

$$
\begin{aligned}
\Pi_{\alpha \beta j}= & -X_{\beta} \mathbb{D}_{\alpha \beta}\left[\left(\frac{m_{\alpha}}{m} \bar{\alpha}_{T, \beta}^{b}-\frac{m_{\beta}}{m} \bar{\alpha}_{T, \alpha}^{b}\right) \frac{1}{T} \frac{\partial T}{\partial x_{j}}+\frac{m_{\alpha} m_{\beta}}{m R_{u} T}\left(\frac{v_{\alpha}}{m_{\alpha}}-\frac{v_{\beta}}{m_{\beta}}\right) \frac{\partial p}{\partial x_{j}}\right] \\
& -\sum_{\gamma=1}^{N-1}\left(\frac{m_{\beta}}{m_{\gamma}} \mathbb{D}_{\beta \alpha} \alpha_{D \alpha \gamma}-\frac{m_{\alpha}}{m_{\gamma}} \mathbb{D}_{\alpha \beta} \alpha_{D \beta \gamma}\right)\left[\frac{\partial Y_{\gamma}}{\partial x_{j}}-Y_{\gamma} \sum_{\delta=1}^{N-1}\left(\frac{m}{m_{\delta}}-\frac{m}{m_{N}}\right) \frac{\partial Y_{\delta}}{\partial x_{j}}\right]
\end{aligned}
$$

with $\Pi_{\alpha \alpha j}=0, \Pi_{\alpha \beta j}=-\Pi_{\beta \alpha j}$. The $g$ form of (6.35) is the multi-species equivalent of the binary-species form provided by Okong'o \& Bellan (2002a). Of note, $g_{\text {temp }}$ is not the same quantity as $g_{\text {heat }}$ since the portion of $\boldsymbol{q}$ containing the enthalpy transported by the species is not contained in $g_{\text {temp }}$ and has instead been apportioned to $g_{\text {mass }}$. Having diagonalized the species conservation equations and provided models for the effective diffusion coefficients in $\S 6.2 .2$, the modelled flux of species $\alpha$ from (6.11) and modelled heat flux from (6.16) are

$$
\begin{aligned}
J_{\alpha j}^{*} & =-\rho \check{D}_{\alpha} \frac{\partial Y_{\alpha}}{\partial x_{j}}, \\
q_{j}^{*} & =-\Lambda \frac{\partial T}{\partial x_{j}},
\end{aligned}
$$

and we model

$$
g_{T}=\left(g_{\text {temp }}+g_{\text {mass }}\right)=\left(g_{\text {heat }}+g_{\text {mass }, \text { flux }}\right)
$$



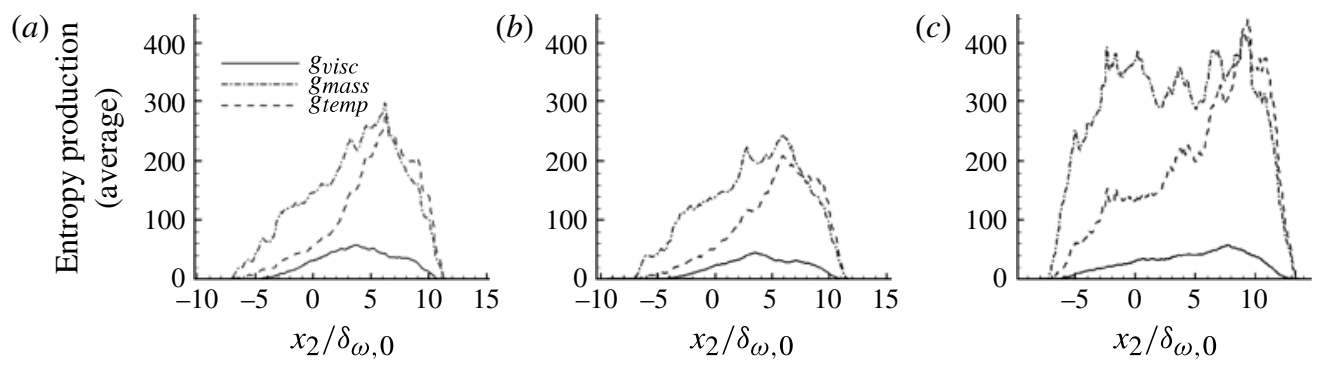

FIGURE 17. Planar averages in $\left(x_{1}, x_{3}\right)$ planes of the three modes, $g_{\text {visc }}, g_{\text {temp }}$ and $g_{\text {mass }}$ of the irreversible entropy production $g$, for R1000p80 (a), R1000p60 (b) and R2000p60 (c), all at the respective $t_{t r}^{*}$. The values of the transport coefficients are not $\mathscr{F}$-scaled and units are $\mathrm{W}\left(\mathrm{m}^{3} \mathrm{~K}\right)^{-1}$.

as

$$
g_{T}^{*}=\underbrace{\Lambda \frac{1}{T^{2}} \frac{\partial T}{\partial x_{j}} \frac{\partial T}{\partial x_{j}}}_{\text {g }_{\text {heat }}^{*}}+\underbrace{\rho R_{u} \sum_{\alpha=1}^{N} \frac{\check{D}_{\alpha}}{m_{\alpha} Y_{\alpha}} \frac{\partial Y_{\alpha}}{\partial x_{j}} \frac{\partial Y_{\alpha}}{\partial x_{j}}}_{\text {s }_{\text {mass.flux }}^{*}} .
$$

Notably, $\check{D}_{\alpha}$ has been defined and computed for only four out of the five species since the equations were written for only an independent number of species by eliminating the conservation equation for $\mathrm{N}_{2}$. To obtain the necessary quantities to compute the $\mathrm{N}_{2}$ contribution in the last term of (6.40), we used the flux conservation $\sum_{\alpha=1}^{N} J_{\alpha j}^{*}=0$ to compute $\check{D}_{N}\left(\partial Y_{N} / \partial x_{j}\right)$, mass conservation $\sum_{\alpha=1}^{N} Y_{\alpha}=1$ to compute $Y_{N}$, and the gradient of the mass conservation $\nabla\left(\sum_{\alpha=1}^{N} Y_{\alpha}\right)=0$ to compute $\partial Y_{N} / \partial x_{j}$ all of which are utilized to calculate the $\mathrm{N}_{2}$ contribution in the summation of (6.40). In (6.40), $g_{\text {heat }}^{*}$ is a functional model of $g_{\text {heat }}$ but $g_{\text {mass,fux }}^{*}$ is not a functional model of $g_{\text {mass }, f u x}$.

Because $\check{D}_{\alpha}$ and $\Lambda$ assume both positive and negative values, as seen in $\$ 6.2 .2 .4$ and $\$ 6.2 .2 .5$, the form of equation (6.40) does not necessarily indicate positivity for $g_{T}^{*}$; indeed, the sign of $g_{T}^{*}$ is determined from other considerations. The contribution to $g$ given by $g_{T}$ as defined by (6.39) is such that $g_{T} \geqslant 0$ since $g_{T}$ is the sum of quadratic terms. Thus, it may be expected that $g_{T}^{*} \geqslant 0$ although there is no such expectation for its individual contributions. Examination of (6.25)-(6.27) shows that because the denominator in each expression has a quadratic form which is also encountered in $g_{T}^{*}$, the modelled coefficients are in fact exact in $g_{T}^{*}$. As a consequence, the contribution $g_{\text {heat }}^{*}$ based on $q_{j}^{*}$ (6.38) which uses exact coefficients is a 'reformulated' form of $g_{\text {heat }}$ rather than a model. Instead, although also $g_{\text {mass, fux }}^{*}$ uses exact coefficients, it represents only a heuristic model of $g_{\text {mass, fux }}$; this is in direct contrast to the derivation in $\S 6.2 .2 .3$ where the fluxes were exact but the coefficients entered the computations of these fluxes as an approximation through the model. To inquire whether the model of $\S 6.2 .2$ preserves positivity of the irreversible entropy production and is accurate, $g, g_{T}$ and $g_{T}^{*}$ are computed, analysed and compared.

Illustrated in figure 17 are $g_{\text {visc }}, g_{\text {temp }}$ and $g_{\text {mass }}$ for R1000p60, R1000p80 and R2000p60 computed as $\left(x_{1}, x_{3}\right)$ plane averages. Similar to the binary-species results of Okong'o \& Bellan (2002a), $g_{\text {mass }}$ is the largest contribution to $g$ while $g_{v i s c}$ is the smallest. But in contrast to the findings of Okong'o \& Bellan (2002a) for binary 

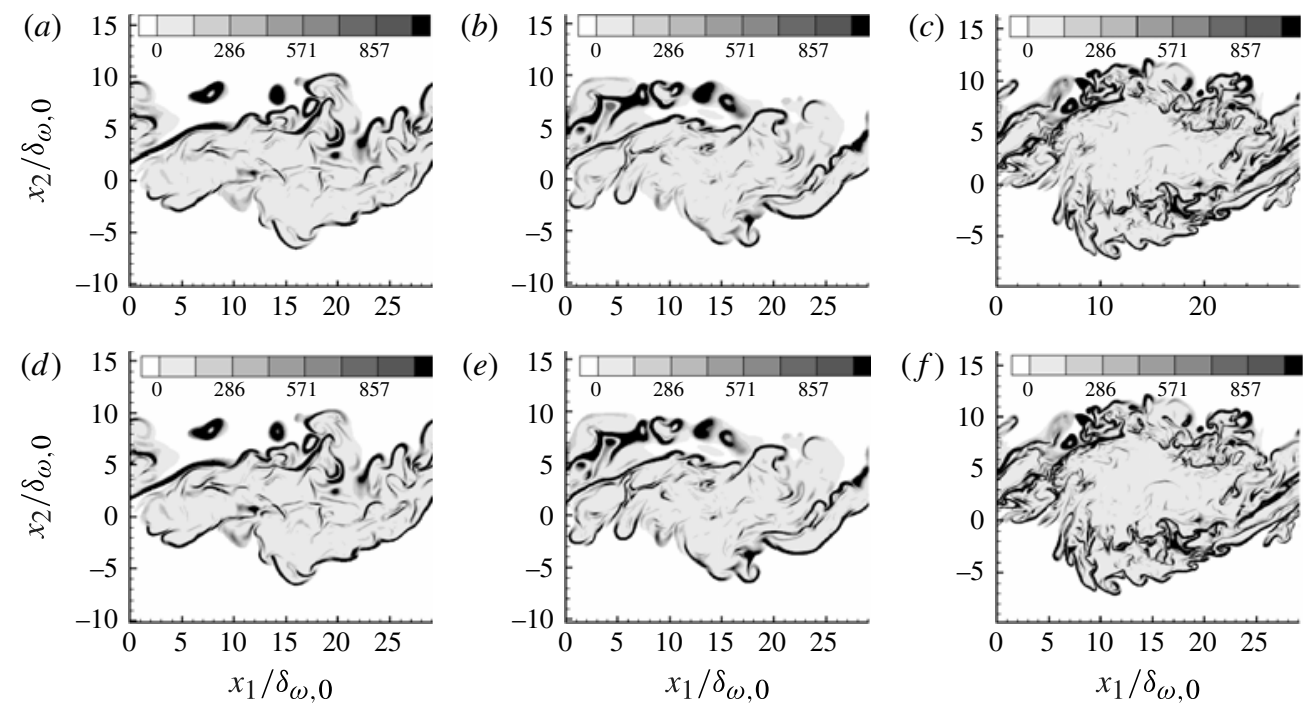

FIGURE 18. $g_{T}(a-c)$ and $g_{T}^{*}(d-f)$ for R1000p80 $(a, d), \mathrm{R} 1000 \mathrm{p} 60(b, e)$ and R2000p60 $(c, f)$ in the $x_{3} / L_{3}=1 / 16$ plane, all at the respective $t_{t r}^{*}$. The values of the transport coefficients are not $\mathscr{F}$-scaled and units are $\mathrm{W}\left(\mathrm{m}^{3} \mathrm{~K}\right)^{-1}$.

species, now $g$ is no longer totally dominated by $g_{\text {mass }}$ because $g_{\text {temp }}$ is almost similar in magnitude to $g_{\text {mass }}$. Either larger $p_{0}$ or higher $R e_{0}$ result in an increase in the dissipation. For larger $p_{0}$, this increased is conjectured to be the result of the increased non-ideality of the mixture which produces more prominent $|\nabla \rho|$ regions and thus larger gradients. For higher $R e_{0}$, this increase is due to both a wider range of small scales and more energy in the small scales as illustrated in figure 2.

Since the computation of the product between the coefficients modelled using (6.25)-(6.27) and the respective quadratic form of the gradients is exact in $g_{T}^{*}$, a comparison between $g_{T}$ and $g_{T}^{*}$ is a suitable indicator of the accuracy of the model of $g_{T}$ through $g_{T}^{*}$. Visualizations of $g_{T}$ and $g_{T}^{*}$ are displayed in figure 18 for R1000p60, R1000p80 and R2000p60 showing that independent of the simulation, the model of $g_{T}$ through $g_{T}^{*}$ is excellent. For larger $R e_{0}$, the high dissipation regions have a more filamentary and more widely distributed aspect. The variation of these high-dissipation regions with $p_{0}$ change is less distinctive. In all cases, the dissipation is very large in the stoichiometric regions and in regions enclosed by stoichiometric boundaries, this coincidence being a manifestation of intense mixing. Regions having $T$ values below the minimum initial temperature experience very high dissipation (compare figure $18 a-c$ with figure $12 a-c$ ) indicating that $T$ would have decreased even further were it not for the heat provided by the dissipation.

A more quantitative evaluation of how well the model replicates the exact dissipation is presented through conditional averages in figure 19, first comparing the modelling of $g_{\text {heat }}$ by $g_{\text {heat }}^{*}$ and then the modelling of $g_{T}$ by $g_{T}^{*}$ over the entire mixing layer rather than in a single $x_{3}$ plane as in figure 18. The vertical line in figure $19(d-f)$ indicates the mean over the mixing layer. Clearly, as expected, $g_{\text {heat }}^{*}$ exactly reproduces $g_{\text {heat }}$ over the entire range of its values as the conditional average essentially coincides with the $45^{\circ}$-slope line. Regarding $g_{T}^{*}$, it represents $g_{T}$ very accurately at and around the mean, but as the values deviate more substantially from the mean, the heuristic 

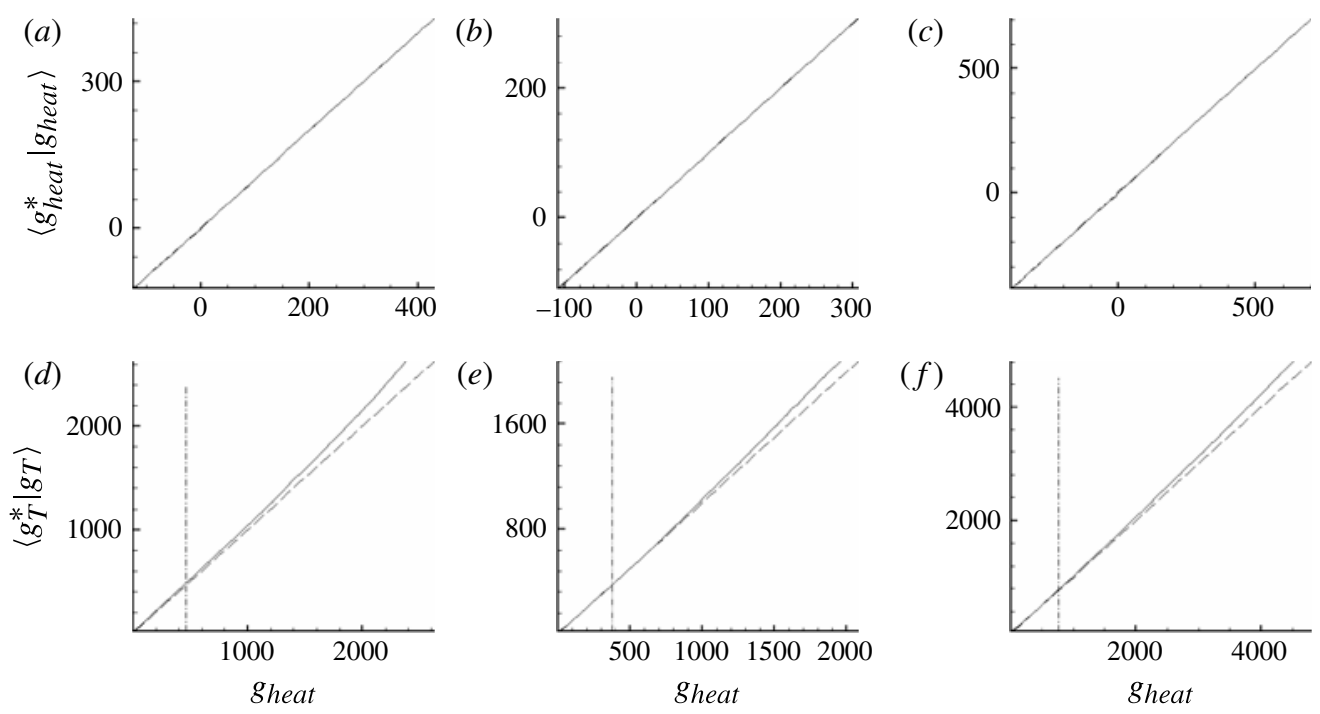

FIGURE 19. Conditional averages of $g_{\text {heat }}^{*}$ with respect to $g_{\text {heat }}(a-c)$ and $g_{T}^{*}$ with respect to $g_{T}(d-f)$ represented by the solid line: R1000p80 $(a, d), \mathrm{R} 1000 \mathrm{p} 60(b, e)$ and R2000p60 $(c, f)$, all computed into the mixing layer $\left(0.01 \leqslant Y_{\text {Hep }} \leqslant 0.945\right)$ at $t_{t r}^{*}$. The dashed line represents the $45^{\circ}$ slope (perfect agreement). The evaluation is performed in the range of \pm 2 times the standard deviation from the mean value. The vertical line in $(d-f)$ represents the value of the mean over the entire domain. The values of the transport coefficients are not $\mathscr{F}$-scaled and units are $\mathrm{W}\left(\mathrm{m}^{3} \mathrm{~K}\right)^{-1}$.

model for $g_{\text {mass }, f u x}$ represented by $g_{\text {mass }, f u x}^{*}$ increasingly overestimates the exact value; however, the deviation of the value obtained using the model from the exact value is very small. Either an increase in $p_{0}$ or in $R e_{0}$ results in a marginally perceptible loss of $g_{T}^{*}$ accuracy that is slightly more pronounced for increasing $p_{0}$ rather than $R e_{0}$.

We conjecture that the very good agreement between $g_{T}^{*}$ and $g_{T}$ is here due to the combined effect of the exact coefficient values in the calculation of $g_{T}^{*}$, and of $g_{T}^{*}$ generally being a reasonable functional model for $g_{T}$. We also note that the species for which $\check{D}_{\alpha}$ may be negative have small gradients and have thus small speciesmass fluxes as compared with the other species; these species-mass fluxes are small because the species undergoing spinodal decomposition must initially be distributed over the entire domain rather than segregated in one stream. The indication is that the dissipation will be here smaller than average in regions of spinodal decomposition. The species-mass flux is much larger if a species is initially segregated in one stream, but in that case the species does not undergo spinodal decomposition, as discussed in $\S 6.2 .2 .6$, and therefore $\check{D}_{\alpha}$ will be positive. Therefore, the species dissipation (usually called scalar dissipation) has an inherent mechanism to maintain positivity. Because positivity of the heat dissipation is entirely determined by the sign of $\Lambda$, an equivalent argument cannot be made for the heat dissipation.

\section{Summary and conclusions}

A modelling-and-simulation study of multi-species mixing under supercritical pressure has been conducted to identify the salient phenomena relevant to the cold ignition regime in Diesel, HCCI and gas turbine engines. The model encompasses 
the differential conservation equations with a complete flux matrix including Soret and Dufour effects, a real-gas equation of state and accurate transport properties (mass-diffusivity matrix, thermal-diffusion matrix, thermal conductivity and viscosity) computed using mixing rules. For supercritical pressure flows, the mixing rules couple molecular transport phenomena with thermodynamics. The configuration for all DNSs was that of a three-dimensional mixing layer and the DNSs followed, for each realization, the evolution of the layer from an initial laminar state to a transitional state. Initially, the lower stream of the layer was composed of heptane vitiated with water and carbon dioxide and the upper stream was composed of nitrogen, oxygen, water and carbon dioxide; the amount of water and carbon dioxide was parametrically varied among simulations. The other two parameters varied among simulations were the initial pressure and initial Reynolds number. The eight DNS realizations thus obtained presented a comprehensive view of multi-species supercritical-pressure mixing.

A general conclusion is that at the Reynolds $(R e)$ number values achievable in DNSs, the smallest flow scales are determined by the variation of scalars (i.e. the species mass fraction and temperature; i.e. the thermodynamics and diffusion) rather than by the motion (i.e. the dynamics) of the flow. We reached this conclusion by reformulating the complex form of the fluxes in the species and energy conservation equations to obtain effective species-specific diffusivities and an effective thermal conductivity which allowed the corresponding definition of effective species-specific Schmidt $(S c)$ numbers and an effective Prandtl $(P r)$ number. Because the reformulation involved quantities not directly computable from the DNS database, models were developed to calculate these effective species-specific diffusivities and the effective thermal conductivity. The models were evaluated by comparison with the database and proved to be very accurate. When water and carbon dioxide were initially present in both lower and upper stream, with each species not necessarily in equal quantities in the two streams, spinodal decomposition of these two species was obtained. The diagnostic of spinodal decomposition for these species was the existence of regions of negative effective diffusivity, meaning that diffusion occurs in counter gradient fashion; that is, these species segregated into regions of lower and higher contiguous concentrations. To the best of the authors' knowledge, this is the first time that species spinodal decomposition has been identified in: (a) a three-dimensional, turbulent flow and $(b)$ a multi-species (i.e. more than two species) mixture having initially non-uniform chemical composition and in which only some of the species experience spinodal decomposition. We showed that a necessary and sufficient condition for species spinodal decomposition occurrence is that the particular species be initially present in both streams; this is why heptane, nitrogen and oxygen never experienced spinodal decomposition during the physical time of the simulation. We also found that there is no requirement that each species undergoing spinodal decomposition be initially uniformly distributed, as it is usually specified in the literature (Nauman \& He 2001); in fact, non-uniformity will promote, through gradients, the diffusion causing spinodal decomposition. Because chemicalcomposition uniformity is not a requirement for species spinodal decomposition, we conjecture that the high-density-gradient magnitude regions observed even when species were initially segregated, are the precursors to eventual spinodal decomposition in the mixture which is formed by molecular and turbulent processes undergone by the initially segregated species, i.e. this formed mixture represents the initial condition for which spinodal decomposition may occur.

The effective $S c$ number values exceeded 1.8 for heptane in regions of stoichiometric conditions, these being values significantly larger than those for 
gases, but still much smaller than those for liquids which are $O\left(10^{2}\right)$. Equivalent computations of the effective thermal conductivity revealed that it is negative over substantial portions of the layer and the negative values were shown to originate from the enthalpy transported by the species. The effective $\operatorname{Pr}$ values were as large as and exceeding 5, with many portions of the mixing layer displaying values in the range 1-5 that is similar to the $\operatorname{Pr}$ values of 4-5 of R-12 which is a liquid refrigerant. Increasing the initial value of the Reynolds number had small quantitative effect on these non-ideal mixture characteristics, but increasing the initial pressure exacerbated these effects, as expected. Although the illustrations were overwhelmingly presented at the transitional states, these flow characteristics were observed throughout the flow evolution.

We also found that flows experiencing species spinodal decomposition display amplified turbulent aspects compared with those that do not. The amplified turbulence was traced to the enhanced high-gradient regions which form in the process of species mixing by the species segregation; these gradients act akin to solid boundaries in promoting turbulence production within the flow. Because species spinodal decomposition inherently hinders mixing through species segregation but promotes higher turbulence which enhances mixing, the final mixing outcome is a complex combination of these two counteracting effects and cannot be simply predicted without appropriate simulations.

The stoichiometric region was shown to be thin and located at the upper boundary of the mixing layer where it encounters the upper stream. The thin aspect of the stoichiometric region indicates that if this aspect prevails in supercritical-pressure reactive flow, the flame can be classified as a flamelet. However, because the effective species-specific Lewis number was larger than unity in the stoichiometric region, we must await results from a corresponding reactive-flow DNS before making a pronouncement as to applicability of the flamelet models of Peters (2000) for supercritical-pressure combustion. The stoichiometric region was also shown to be surrounded by lean and rich regions which coexist in close proximity to each other.

Calculations of the dissipation showed that among the three contributions, viscous, heat and species diffusion, the largest portion came from the species and the smallest from viscous effects. The sum of the heat and species contribution were functionally modelled to be calculable using the effective species-specific diffusivities and the effective thermal conductivity. This functionally modelled portion of the dissipation computed with the modelled effective species-specific diffusivities and the effective thermal conductivity was shown to accurately duplicate the exact equivalent dissipation.

A thorough comparison (made in appendix $\mathrm{C}$ ) between the diagonal elements of the species mass-diffusion matrix and the corresponding effective species-specific diffusion coefficients elucidated the crucial effect of the off-diagonal terms of the species mass-diffusion matrix, all of which are by a factor of $O\left(10^{1}\right)-O\left(10^{2}\right)$ smaller than the diagonal terms. If one were to neglect these off-diagonal terms, then: $(a)$ a major portion of the diffusivity small-scale structure in the mixing layer would be missed; $(b)$ no species spinodal decomposition would be observed for the species for which it occurs; $(c)$ the qualitative aspect of diffusion would be distorted from reality (i.e. incorrect placement of high- and low-diffusivity regions) for a species as important as oxygen; and $(d)$ the magnitude of the diffusivity for light species (e.g. oxygen) could be in error by a factor of two implying a correspondingly erroneous mixture ignition delay time. Clearly, without the off-diagonal terms, the physics of the problem would be entirely missed. Therefore, we caution about utilizing 
the popular zeroth-order approximation of the diffusion matrix which describes the diffusion of each species into the mixture (Hirshfelder \& Curtis 1949; Giovangigli 1991) and results in a diagonal matrix which not only would be entirely inadequate for portraying small-scale molecular mixing but would additionally be unable to describe species spinodal decomposition because all of the matrix elements are positive. Of note, Ern \& Giovangigli (1994) state that a diagonal approximation of the diffusion matrix is only appropriate if the diagonal elements are equal. Mathematically, the importance of the small off-diagonal terms is consistent with asymptotic expansion concepts wherein the crucial behaviour is embodied in a small parameter.

Because transport properties operate at small scales unresolved in LES, the importance of modelling the flow using accurate effective transport properties indicated the need for correspondingly appropriate subgrid-scale models in LES to describe these small-scale processes.

The general methodology introduced here will be extended to supercritical-pressure reactive-flow DNSs to investigate whether the conclusions obtained from this mixing study also hold for reactive flows. Projecting the present findings to the pyrolysis reaction regime which is the precursor to ignition, we can foresee that species formed during pyrolysis will be distributed by the turbulent flow over the entire field, at which point their spinodal decomposition will be eventually inevitable unless they are consumed by reaction faster than they are transported by turbulence or that turbulence is so vigorous as to impede spinodal decomposition.

\section{Acknowledgements}

This work was conducted at the Jet Propulsion Laboratory (JPL) of the California Institute of Technology (Caltech) and sponsored by the Department of Energy (DOE), Basic Energy Sciences (BES) under the direction of Dr W. Sisk and Dr M. Pederson. Sponsorship from the JPL Research and Technology Development under the Spontaneous Concepts program permitted the development of some ideas which were fully explored under the DOE-BES sponsorship. The computational resources were provided by the JPL Supercomputing Center, by NASA Advanced Supercomputing at Ames Research Center and by National Energy Research Supercomputing Center of the Department of Energy.

\section{Appendix A. Relationships for the EOS}

Miscellaneous relationships relevant to the EOS are

$$
a_{m i x}=\sum_{\alpha} \sum_{\gamma} X_{\alpha} X_{\gamma} a_{\alpha \gamma}(T), \quad b_{m i x}=\sum_{\alpha} X_{\alpha} b_{\alpha},
$$

where indices do not follow the Einstein notation, and

$$
\begin{gathered}
a_{\alpha \gamma}=\left(1-k_{\alpha \gamma}^{\prime}\right) \sqrt{\alpha_{\alpha \alpha} \alpha_{\gamma \gamma}}, \\
\alpha_{\alpha \alpha}(T) \equiv 0.457236\left(R_{u} T_{c, \alpha}\right)^{2} \frac{\left[1+c_{\alpha}\left(1-\sqrt{T_{r e d, \alpha}}\right]^{2}\right.}{p_{c, \alpha}}, \\
c_{\alpha}=0.37464+1.54226 \Omega_{\alpha}-0.26992 \Omega_{\alpha}^{2},
\end{gathered}
$$


where $T_{\text {red }, \alpha} \equiv T / T_{c, \alpha}, T_{c, \alpha}$ is the critical temperature and $\Omega_{\alpha}$ is the acentric factor. Also,

$$
\begin{gathered}
b_{\alpha}=0.077796 \frac{R_{u} T_{c, \alpha}}{p_{c, \alpha}}, \\
T_{c, \alpha \gamma}=\left(1-k_{\alpha \gamma}\right) \sqrt{T_{c, \alpha} T_{c, \gamma}} \text { with } k_{\alpha \alpha}=0, \\
v_{c, \alpha \gamma}=\frac{1}{8}\left(v_{c, \alpha}^{1 / 3}+v_{c, \gamma}^{1 / 3}\right)^{3}, \\
Z_{c, \alpha \gamma}=\frac{1}{2}\left(Z_{c, \alpha}+Z_{c, \gamma}\right), \\
p_{c, \alpha \gamma}=\frac{R_{u} T_{c, \alpha \gamma} Z_{c, \alpha \gamma}}{v_{c, \alpha \gamma n}},
\end{gathered}
$$

with $T_{r e d, \alpha \gamma} \equiv T / T_{c, \alpha \gamma}, Z_{c, \alpha}$ being the critical compression factor with the compression factor defined as $Z=p /\left(\rho T R_{u} / m\right), v_{c, \alpha}$ being the critical volume and $p_{c, \alpha}$ being the critical pressure. $k_{\alpha \gamma}$ is an empirical mixing parameter. The relationship between parameters $k_{\alpha \gamma}$ and $k_{\alpha \gamma}^{\prime}$ is

$$
\left(1-k_{\alpha \gamma}\right)=\left(1-k_{\alpha \gamma}^{\prime}\right) \frac{\left(v_{c, \alpha} v_{c, \gamma}\right)^{1 / 2}}{v_{c, \alpha \gamma}} .
$$

and for all pairs not in table $4, k_{\alpha \gamma}^{\prime}=0$ is used.

\section{Appendix B. Initial profile derivation}

\section{B.1. Mean profiles}

The mean profiles are only functions of $x_{2}$

$$
\begin{aligned}
u_{1}\left(x_{2}\right) & =u_{1}\left(x_{2, \min }\right)+\left[1+\operatorname{erf}\left(\frac{\sqrt{\pi} x_{2}}{\delta_{\omega, 0}}\right)\right] \frac{1}{2}\left[u_{1}\left(x_{2, \max }\right)-u_{1}\left(x_{2, \min }\right)\right] \\
T\left(x_{2}\right) & =T\left(x_{2, \min }\right)+\left[1+\operatorname{erf}\left(\frac{\sqrt{\pi} x_{2}}{\delta_{\omega, 0}}\right)\right] \frac{1}{2}\left[T\left(x_{2, \max }\right)-T\left(x_{2, \min }\right)\right] \\
Y_{\alpha}\left(x_{2}\right) & =Y_{\alpha}\left(x_{2, \min }\right)+\left[1+\operatorname{erf}\left(\frac{\sqrt{\pi} x_{2}}{\delta_{\omega, 0}}\right)\right] \frac{1}{2}\left[Y_{\alpha}\left(x_{2, \max }\right)-Y_{\alpha}\left(x_{2, \min }\right)\right] \\
p & =p_{\infty}, \quad u_{2}=0, \quad u_{3}=0 \\
\frac{\partial u_{1}}{\partial x_{2}} & =\frac{\left[u_{1}\left(x_{2, \max }\right)-u_{1}\left(x_{2, \min }\right)\right]}{\delta_{\omega, 0}} \exp \left[-\left(\frac{\sqrt{\pi} x_{2}}{\delta_{\omega, 0}}\right)^{2}\right] \\
& =\frac{\Delta U_{0}}{\delta_{\omega, 0}} \exp \left[-\left(\frac{\sqrt{\pi} x_{2}}{\delta_{\omega, 0}}\right)^{2}\right]
\end{aligned}
$$

where $u_{1}\left(x_{2, \max }\right)=U_{1}$ and $u_{1}\left(x_{2, \min }\right)=U_{2}$ where $L_{2}$ is the length of the domain in the $x_{2}$ direction. The values of $U_{1}$ and $U_{2}$ are obtained by invoking a null convective velocity $U_{c}$ computed according to Papamoschou \& Roshko (1988) as

$$
U_{c}=\frac{\sqrt{\rho_{1}} U_{1}+\sqrt{\rho_{2}} U_{2}}{\sqrt{\rho_{1}}+\sqrt{\rho_{2}}} .
$$


For $U_{c}=0$, and defining as the speed of sound,

$$
U_{1}=\frac{2 M_{c} a_{s 1}}{\left(1+\frac{a_{s 1}}{a_{s 2}} \sqrt{\frac{\rho_{1}}{\rho_{2}}}\right)}, \quad U_{2}=-\sqrt{\frac{\rho_{1}}{\rho_{2}}} U_{1} .
$$

\section{B.2. Perturbations}

The simulations are started with streamwise and spanwise vorticity perturbations superimposed on the mean initial velocity profile

$$
\begin{aligned}
& \omega_{1}\left(x_{2}, x_{3}\right)=F_{3 D} \frac{\lambda_{3} \Delta U_{0}}{\Gamma_{1}} f_{2}\left(x_{2}\right) f_{3}\left(x_{3}\right) \\
& \omega_{3}\left(x_{1}, x_{2}\right)=F_{2 D} \frac{\lambda_{1} \Delta U_{0}}{\Gamma_{3}} f_{1}\left(x_{1}\right) f_{2}\left(x_{2}\right)
\end{aligned}
$$

where $\Gamma_{1}$ and $\Gamma_{3}$ are the circulations, and $\lambda_{1}$ and $\lambda_{3}$ are wavelengths

$$
\begin{gathered}
f_{1}\left(x_{1}\right)=A_{k} \sin \left(\frac{2 \pi x_{1}}{2^{k} \lambda_{1}}\right) \\
f_{2}\left(x_{2}\right)=\exp \left[-\pi\left(\frac{x_{2}}{\delta_{\omega, 0}}\right)^{2}\right] \\
f_{3}\left(x_{3}\right)=B_{k} \sin \left(\frac{2 \pi x_{3}}{2^{k} \lambda_{3}}\right) .
\end{gathered}
$$

Unlike for our simulations for binary-species systems (Okong'o \& Bellan 2002a; Okong'o et al. 2002), for each vorticity component $\left(\omega_{1}, \omega_{3}\right)$, the velocity components are computed from the analytical solution of a system consisting of the vorticity component equation

$$
\omega_{1}=\frac{\partial u_{3}}{\partial x_{2}}-\frac{\partial u_{2}}{\partial x_{3}}, \quad \omega_{2}=\frac{\partial u_{1}}{\partial x_{3}}-\frac{\partial u_{3}}{\partial x_{1}}, \quad \omega_{3}=\frac{\partial u_{2}}{\partial x_{1}}-\frac{\partial u_{1}}{\partial x_{2}}
$$

and the divergence-free condition. To ensure divergence-free initial conditions, the analytical perturbation is obtained separately for each wavelength $\left(2^{k} \lambda_{1}\right.$ and $\left.2^{k} \lambda_{3}\right)$ and then the perturbations are added together after being weighted according to $A_{k}$ or $B_{k}$. The analytical perturbation at a given wavelength is separately derived for the spanwise and streamwise directions.

Let $u_{i}$ and $\omega_{i}$ be the generic perturbation velocity and perturbation vorticity fields. In a $x_{3}$ plane, for the spanwise vorticity

$$
\begin{aligned}
& \frac{\partial u_{2}}{\partial x_{1}}-\frac{\partial u_{1}}{\partial x_{2}}=\omega_{3} \\
& \frac{\partial u_{1}}{\partial x_{1}}+\frac{\partial u_{2}}{\partial x_{2}}=0 .
\end{aligned}
$$

Introducing wavelength $n \lambda_{1}$ (wavenumber $\alpha_{n}=2 \pi /\left(n \lambda_{1}\right)$ ) we define

$$
\alpha_{n}^{*}=\alpha_{n} \delta_{\omega, 0}, \quad x_{2}^{*}=\frac{x_{2}}{\delta_{\omega, 0}}, \quad \hat{\omega}_{3}^{*}=\hat{\omega}_{3} \delta_{\omega, 0}
$$

and the perturbations

$$
u_{1}=\hat{u}_{1}\left(x_{2}^{*}\right) \exp \left(\mathrm{i} \alpha_{n}^{*} x_{1}^{*}\right), \quad u_{2}=\hat{u}_{2}\left(x_{2}^{*}\right) \exp \left(\mathrm{i} \alpha_{n}^{*} x_{1}^{*}\right) \quad \omega_{3}=\hat{\omega}_{3}\left(x_{2}^{*}\right) \exp \left(\mathrm{i} \alpha_{n}^{*} x_{1}^{*}\right)
$$


where $\hat{u}_{1}, \hat{u}_{2}$ and $\hat{\omega}_{3}$ are the perturbation amplitudes. Solving (B 14) and (B 15) for the form of (B 17) and applying far-field boundary conditions $\hat{u}_{2}\left(x_{2, \min }\right)=0$ and $\hat{u}_{2}\left(x_{2, \max }\right)=0$ yields

$$
\begin{aligned}
& \hat{u}_{1}=\mathrm{i} a_{1} \exp \left(\alpha_{n}^{*} x_{2}^{*}\right)-\mathrm{i} a_{2} \exp \left(-\alpha_{n}^{*} x_{2}^{*}\right), \\
& \hat{u}_{2}=a_{1} \exp \left(\alpha_{n}^{*} x_{2}^{*}\right)+a_{2} \exp \left(-\alpha_{n}^{*} x_{2}^{*}\right),
\end{aligned}
$$

where

$$
\begin{aligned}
& a_{1}=\mathrm{i} \frac{1}{4} \exp \left[\left(\frac{\alpha_{n}^{*}}{2 \sqrt{\pi}}\right)^{2}\right]\left[\operatorname{erf}\left(\sqrt{\pi} x_{2}^{*}+\frac{\alpha_{n}^{*}}{2 \sqrt{\pi}}\right)-1\right], \\
& a_{2}=-\mathrm{i} \frac{1}{4} \exp \left[\left(\frac{\alpha_{n}^{*}}{2 \sqrt{\pi}}\right)^{2}\right]\left[\operatorname{erf}\left(\sqrt{\pi} x_{2}^{*}-\frac{\alpha_{n}^{*}}{2 \sqrt{\pi}}\right)^{2}+1\right] .
\end{aligned}
$$

A similar solution is obtained for the streamwise vorticity perturbation by solving the equations

$$
\begin{aligned}
& \frac{\partial u_{2}}{\partial x_{3}}-\frac{\partial u_{3}}{\partial x_{2}}=-\omega_{1}, \\
& \frac{\partial u_{3}}{\partial x_{3}}+\frac{\partial u_{2}}{\partial x_{2}}=0,
\end{aligned}
$$

in a $x_{1}$ plane with boundary conditions $\hat{u}_{2}\left(x_{2, \min }\right)=0$ and $\hat{u}_{2}\left(x_{2, \max }\right)=0$. Formally, we replace $x_{1}$ by $x_{3}, \lambda_{1}$ by $\lambda_{3}$ (the relevant wavelength is $n \lambda_{3}$ (wavenumber $\left.\left.\alpha_{n}=2 \pi /\left(n \lambda_{3}\right)\right)\right), u_{1}$ by $u_{3}$ and $\omega_{3}$ by $\left(-\omega_{1}\right)$. The final result is

$$
\begin{aligned}
& \hat{u}_{3}=\mathrm{i} a_{1} \exp \left(\alpha_{n}^{*} x_{2}^{*}\right)-\mathrm{i} a_{2} \exp \left(-\alpha_{n}^{*} x_{2}^{*}\right) \\
& \hat{u}_{2}=a_{1} \exp \left(\alpha_{n}^{*} x_{2}^{*}\right)+a_{2} \exp \left(-\alpha_{n}^{*} x_{2}^{*}\right)
\end{aligned}
$$

with the same formal expressions (B 20) and (B 21) for $a_{1}$ and $a_{2}$, but where $\alpha_{n}^{*}$ is now related to $\lambda_{3}$ instead of to $\lambda_{1}$.

\section{Appendix C. Further considerations on diffusion and effective-diffusion coefficients}

We wish to examine here several aspects that are of interest for mixing and combustion simulation. First, since the matrix of diffusion coefficients $\mathfrak{D}_{\alpha \beta}$ is diagonally dominant with the elements off-diagonal being by $O(10)-O\left(10^{2}\right)$ smaller than the diagonal ones, it would be tempting to assume that the off-diagonal terms are null and conduct calculations using a diagonal matrix. We inquire whether the physics of the situation would be preserved by this assumption. Second, a common assumption in combustion models is that $\rho \mathfrak{D}_{\alpha \beta}$ is a spatially independent quantity. We evaluate this assumption by visualizing $\rho \mathfrak{D}_{\alpha \alpha}$; results for R2000p60 are displayed in figure 20 where we also illustrate $\rho \check{D}_{\alpha}$ to inquire if it could be considered uniform. Assessment of the $\rho \mathfrak{D}_{\alpha \alpha}$ and $\rho \check{D}_{\alpha}$ uniformity indicates here the accuracy of this assumption for mixing studies. Nevertheless, the validity of the results is expected to only change quantitatively rather than qualitatively for combustion applications; however, a more definitive assessment will be made in the future using a reactive-flow database. 

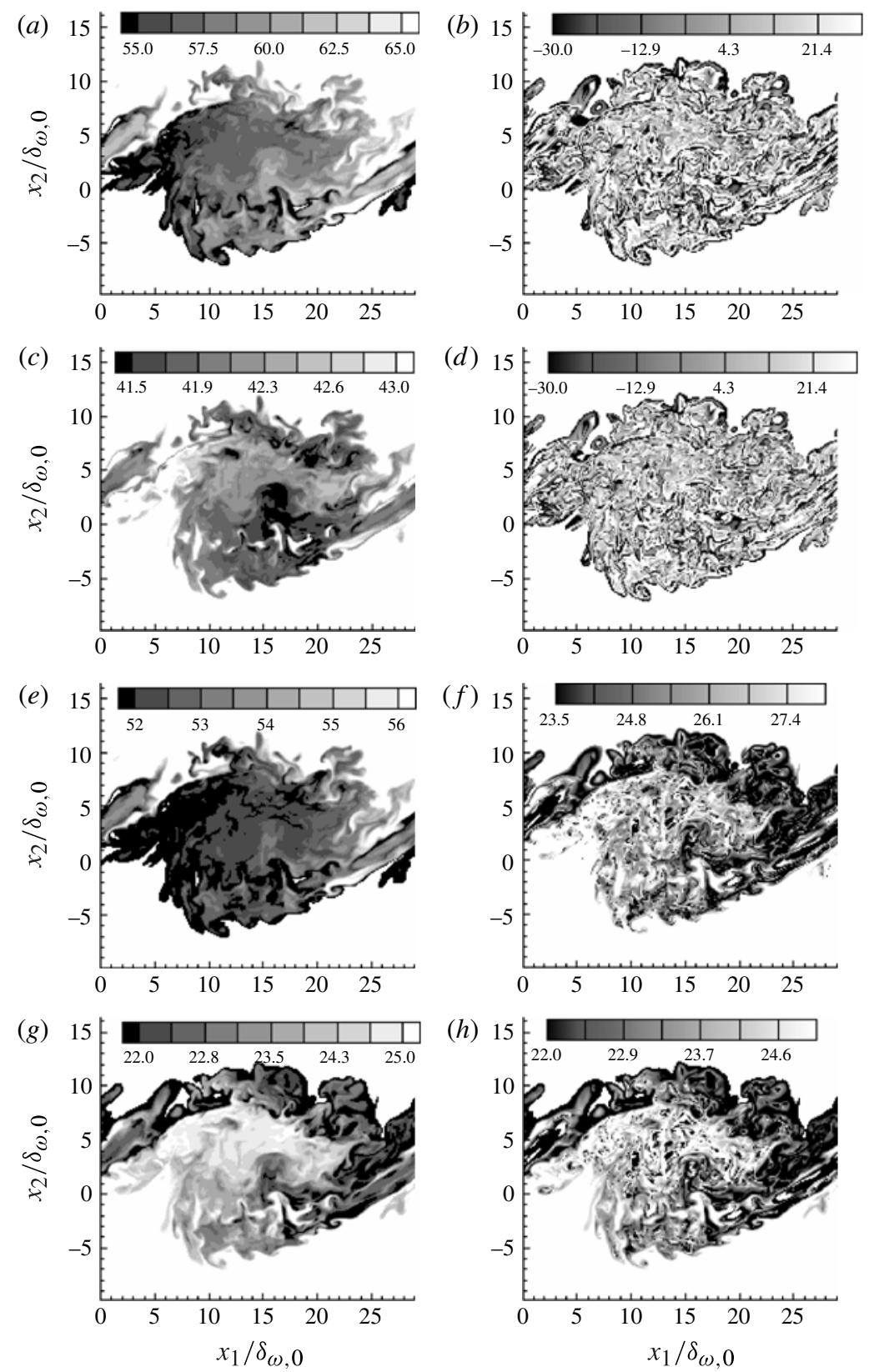

FIGURE 20. Diagonal elements $\rho \mathfrak{D}_{\alpha \alpha}$ of the species mass-diffusion matrix $(a, c, e, g)$ and $\rho \check{D}_{\alpha}$ $(b, d, f, h)$ for $\mathrm{H}_{2} \mathrm{O}(a, b), \mathrm{CO}_{2}(c, d), \mathrm{O}_{2}(e, f)$ and $\mathrm{C}_{7} \mathrm{H}_{14}(g, h)$, all for $\mathrm{R} 2000 \mathrm{p} 60$ at $t_{\text {tr }}^{*}$ in the plane $x_{3} / L_{3}=1 / 16$. The mixing layer is defined as the region where $0.01 \leqslant Y_{\text {Hep }} \leqslant 0.945$. Units are $10^{-6} \mathrm{~kg}(\mathrm{~m} \mathrm{~s})^{-1}$.

Third, because the Soret effects are relatively small in these multi-species simulations (not shown), comparing the diagonal term $\rho \mathfrak{D}_{\alpha \alpha}$ with $\rho \check{D}_{\alpha}$ reveals the influence of the off-diagonal terms. 
The illustrations of figure 20 show that the general features of $\rho \mathfrak{D}_{\alpha \alpha}$ are very much species dependent whereas for $\rho \check{D}_{\alpha}$ the behaviours can be categorized in two distinct aspects: that of $\mathrm{H}_{2} \mathrm{O}$ and $\mathrm{CO}_{2}$ (figure 20b,d), and that of $\mathrm{O}_{2}$ and $\mathrm{C}_{7} \mathrm{H}_{16}$ (figure 20f,h). With the exception of $\mathrm{C}_{7} \mathrm{H}_{16}$, for all species $\rho \mathfrak{D}_{\alpha \alpha}$ and $\rho \check{D}_{\alpha}$ have a different spatial variation, a fact which is a clear manifestation of the influence of the off-diagonal terms in the diffusion matrix. For $\mathrm{C}_{7} \mathrm{H}_{16}$, its much larger molar mass makes the diagonal term surpass in magnitude all other terms involved in the computation of $\check{D}_{H e p}$, and thus the large-scale features are very similar for $\rho \mathfrak{D}_{\alpha \alpha}$ and $\rho \check{D}_{\alpha}$. However, even for $\mathrm{C}_{7} \mathrm{H}_{16}$ the small-scale variation observable for $\rho \check{D}_{\alpha}$ is lost when considering the corresponding $\rho \mathfrak{D}_{\alpha \alpha}$. In fact, all $\rho \check{D}_{\alpha}$ show considerably more small-scale structure than the respective $\rho \mathfrak{D}_{\alpha \alpha}$, an aspect which is of great importance for combustion studies because reaction occurs locally according to the mixture composition. Moreover, except for $\mathrm{C}_{7} \mathrm{H}_{16}$, the relative placement of the lowand high-diffusivity regions is different in $\rho \mathfrak{D}_{\alpha \alpha}$ and $\rho \check{D}_{\alpha}$; for example, for $\mathrm{O}_{2}, \rho \mathfrak{D}_{\alpha \alpha}$ indicates that the low-diffusivity regions are where $\rho \check{D}_{\alpha}$ shows high-diffusivity regions, and vice versa. Evaluating the range of $\rho \mathfrak{D}_{\alpha \alpha}$ variation, the conclusion is that this range is rather narrow for each of the species although within that narrow range there is substantial non-uniformity; thus, as a crude approximation, the assumption that $\rho \mathfrak{D}_{\alpha \alpha}$ is uniform may be acceptable. The situation is totally different for $\rho \check{D}_{\alpha}$ where although the range of variation for the major species is still narrow, that for the minor, light species is very wide; therefore, the assumption that $\rho \check{D}_{\alpha}$ is uniform would be catastrophic for the accuracy of a simulation. Finally, comparing the range of variation of $\rho \mathfrak{D}_{\alpha \alpha}$ and $\rho \check{D}_{\alpha}$, it is clear that it is vastly different for all species except $\mathrm{C}_{7} \mathrm{H}_{16}$ for which, as discussed previously, the off-diagonal terms have negligible influence. The conclusion is that not only would the fine structure of the field be missed by neglecting the off-diagonal terms, but also that the quantitative values would be extremely different (e.g. for $\mathrm{O}_{2}$ the significant range of $[52,56]$ for $\rho \mathfrak{D}_{\alpha \alpha}$ and $[24,28]$ for $\rho \check{D}_{\alpha}$, and for $\mathrm{H}_{2} \mathrm{O}$ the significant range of $[55,65]$ for $\rho \mathfrak{D}_{\alpha \alpha}$ and $[-30,30]$ for $\rho \check{D}_{\alpha}$; units are $10^{-6} \mathrm{~kg}(\mathrm{~ms})^{-1}$ ) with major consequences in the prediction of species mixing and thus of the mixture ignition delay time. Since all $\rho \mathfrak{D}_{\alpha \alpha}$ are positive whereas for $\mathrm{CO}_{2}$ and $\mathrm{H}_{2} \mathrm{O}$ the values of $\rho \check{D}_{\alpha}$ are both positive and negative, it is evident that spinodal decomposition would not be obtained if one were to only use the diagonal terms of $\mathfrak{D}_{\alpha \beta}$. This highlights the pivotal role of the off-diagonal terms in describing the correct physics.

\section{REFERENCES}

Balluffi, R. W., Allen, S. M. \& CARTer, W. C. 2005 Kinetics of Materials. John Wiley \& Sons.

Batchelor, G. K. 1999 An Introduction to Fluid Dynamics. Cambridge University Press.

BeLlan, J. 2006 Theory, modelling and analysis of turbulent supercritical mixing. Combust. Sci. Technol. 178, 253-281.

CAHn, J. W. \& Hilliard, J. E. 1958 Free energy of a non-uniform system. I. Interfacial free energy. J. Chem. Phys. 28 (2), 258-267.

DE Groot, S. R. \& MAZUR, P. 1984 Non-equilibrium Thermodynamics. Dover.

Egolfopoulos, F. N. \& Dimotakis, P. E. 1998 Non-premixed hydrocarbon ignition at high strain rates. Proc. Combust. Inst. 27, 641-648.

ERn, A. \& Giovangigli, V. 1994 Multicomponent transport algorithms. In Lecture Notes in Physics Monographs, M 24. Springer. 
ERn, A. \& Giovangigli, V. 1998 Thermal diffusion effects in hydrogen-air and methane-air flames. Combust. Theor. Model. 2, 349-372.

Fotache, C. G., Kreutz, T. G. \& Law, C. K. 1997 Ignition of counterflowing methane versus heated air under reduced and elevated pressures. Combust. Flame 108, 442-470.

Giovangigli, V. 1991 Convergent iterative methods for multicomponent diffusion. Impact Comput. Sci. Engng 3, 244-276.

Giovangigli, V., Matuszewsky, L. \& Dupoirieux, F. 2011 Detailed modelling of planar transcritical $\mathrm{H}_{2}-\mathrm{O}_{2}-\mathrm{N}_{2}$ flames. Combust. Theor. Model. 15, 141-182.

Giovangigli, V. \& MAtUszewsky, L. 2012 Numerical simulation of transcritical strained laminar flames. Combust. Flame 159, 2829-2840.

Goto, S. \& KIDA, S. 1999 Passive scalar spectrum in isotropic turbulence: prediction by the Lagrangian direct-interaction approximation. Phys. Fluids 11 (7), 1936-1952.

Hannoun, I. A., Fernando, H. J. S. \& List, E. J. 1988 Turbulence structure near a sharp density interface. J. Fluid Mech. 189, 189-209.

Harstad, K. \& Bellan, J. 1999 The Lewis number under supercritical conditions. Intl J. Heat Mass Transfer 42, 961-970.

Harstad, K. \& Bellan, J. $2004 a$ Mixing rules for multicomponent mixture mass diffusion coefficients and thermal diffusion factors. J. Chem. Phys. 120 (12), 5664-5673.

HaRstad, K. \& Bellan, J. $2004 b$ High-pressure binary mass-diffusion coefficients for combustion applications. Ind. Engng Chem. Res. 43 (2), 645-654.

Hirshfelder, J. \& CurTis, C. 1949 Theory of propagation of flames. Part I. General equations. In Third Symposium (International) on Combustion, pp. 121-127. The Williams \& Wilkins Co.

Hirshfelder, J., Curtis, C. \& BIRD, R. 1964 Molecular Theory of Gases and Liquids. John Wiley and Sons.

KeIZER, J. 1987 Statistical Thermodynamics of Nonequilibrium Processes. Springler.

Kennedy, C. \& CARPEnter, M. 1994 Several new numerical methods for compressible shear layer simulations. Appl. Numer. Maths 14, 397-433.

Moser, R. \& Rogers, M. 1991 Mixing transition and the cascade to small scales in a plane mixing layer. Phys. Fluids A 3 (5), 1128-1134.

Muller, S. M. \& SCheerer, D. 1991 A method to parallelize tridiagonal solvers. Parallel Comput. 17, 181-188.

Nauman, E. B \& He, D. Q. 2001 Nonlinear diffusion and phase separation. Chem. Engng Sci. 56, 1999-2018.

OKONG'O, N. \& Bellan, J. $2002 a$ Direct numerical simulation of a transitional supercritical binary mixing layer: heptane and nitrogen. J. Fluid Mech. 464, 1-34.

OKong'o, N., Harstad, K. \& Bellan, J. 2002 Direct numerical simulations of $\mathrm{O}_{2} / \mathrm{H}_{2}$ temporal mixing layers under supercritical conditions. AIAA J. 40 (5), 914-926.

OKOnG'O, N. \& Bellan, J. 2002b Consistent boundary conditions for multicomponent real gas mixtures based on characteristic waves. J. Comput. Phys. 176, 330-344.

OKong'o, N. \& Bellan, J. 2004 Perturbation and initial Reynolds number effects on transition attainment of supercritical mixing layers. Comput. Fluids 33 (8), 1023-1046.

Papamoschou, D. \& Roshio, A. 1988 The compressible turbulent shear layer: an experimental study. J. Fluid Mech. 197, 453-477.

Peters, N. 2000 Turbulent Combustion. Cambridge University Press.

Pine, D. J., Easwar, N., Maher, J. V. \& GoldburG, W. I. 1984 Turbulent suppression of spinodal decomposition. Phys. Rev. A. 29 (1), 308-313.

Pitsch, H. \& Peters, N. 1998 Investigation of the ignition process of sprays under diesel engine conditions using n-heptane chemistry. SAE paper 98-2464.

Pope, S. B. 2000 Turbulent Flows. Cambridge University Press.

Rehfeldt, S. \& Stichlmair, J. 2007 Measurement and calculation of multicomponent diffusion coefficients in liquids. Fluid Phase Equilib. 256, 99-104.

Rehfeldt, S. \& Stichlmair, J. 2010 Measurement and prediction of multicomponent diffusion coefficients in four ternary liquid systems. Fluid Phase Equilib. 290, 1-14. 
Reid, R. C., Prausnitz, J. M. \& Polling, B. E. 1987 The Properties of Gases and Liquids, 4th edn. McGraw-Hill.

Selle, L. C., Okong'o, N. A., Bellan, J. \& Harstad, K. G. 2007 Modelling of subgrid scale phenomena in supercritical transitional mixing layers: an a priori study. J. Fluid Mech. 593, 57-91.

Tennekes, H. \& Lumley, J. L. 1989 A First Course in Turbulence. MIT.

Williams, F A. 1975 Turbulent mixing in non-reactive and reactive flows. In Recent Advances in the Theoretical Descriptions of Turbulent Diffusion Flames (ed. S. Murthy). pp. 189-208. Plenum. 